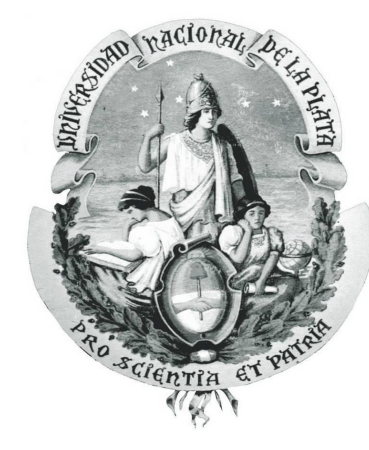

Universidad Nacional de La Plata

Facultad de Ciencias Exactas

Departamento de Matemática

\title{
MODELOS DE CONTENCIÓN: \\ FAMILIAS DE CAMINOS EN UN ÁRBOL
}

\author{
Tesista: \\ Noemí Amalia Gudiño \\ Directora: \\ Dra. Liliana Alcón \\ Tesis para obtener el título de \\ DOCTOR DE LA FACULTAD DE CIENCIAS EXACTAS, AREA MATEMÁTICA
}

Año: 2016 
A mi mamá

Ati,

y a mi papá

Jesús. 


\section{Agradecimientos}

A Liliana y Marisa por su apoyo y dedicación en el desarrollo de este trabajo, por estar cuando las necesité, por el aporte de su experiencia y conocimiento.

Al CONICET, por haberme provisto de una Beca de Posgrado, sin la cual este trabajo no habría sido posible.

A todos los que trabajan en el Departamento de Matemática de la Facultad de Ciencias Exactas de la Universidad Nacional de La Plata, por acompañarme y ayudarme a desarrollar las tareas correspondientes a este trabajo.

A mis amigas Gise y Gaby, y sus respectivas familias, por hacerme sentir como una más de la familia y por tanto cariño.

A mis hermanos, David y Nancy. Por el amor incondicional que me muestran diariamente, y por hacerme reír.

A mi mamá Ati, y a mi papá Jesús. Por acompañarme y ayudarme siempre. 


\section{Contenido}

1. Introducción 1

2. Preliminares 4

2.1. Grafos .......................... . . . 4

2.2. Posets . . . . . . . . . . . . . . . . . . 7

2.3. Grafos de comparabilidad, posets y modelos por contención . . . . . . 9

3. Vértices extremos de grafos $C I \quad 19$

3.1. Caracterización de vértices extremos . . . . . . . . . . . . . . . . 19

3.2. Grafos CI homogéneamente representables . . . . . . . . . . . . 26

4. Posets CPT: Propiedades Generales y Dimensión 27

4.1. Propiedades Generales . . . . . . . . . . . . . . . 27

4.2. Subclases . . . . . . . . . . . . . . . . . . . . . . . 38

4.3. Dimensión de los posets $C P T \ldots \ldots$. . . . . . . . 40

5. Posets y grafos $C P T$ en la clase Split $\quad 45$

5.1. Posets Split . . . . . . . . . . . . . . . . . 45

5.2. Grafos CPT Split . . . . . . . . . . . . . . . . . 51

6. Posets y grafos $C P T$ en la clase $k$-tree $\quad \mathbf{5 4}$

6.1. Posets 2-tree . . . . . . . . . . . . . . . . . . 55

6.2. Posets $k$-tree . . . . . . . . . . . . . . . . . . 65

6.3. Grafos CPT $k$-trees . . . . . . . . . . . . . . . 80

7. Conclusiones y trabajo futuro $\quad 83$

$\begin{array}{lr}\text { Bibliografía } & 87\end{array}$ 


\section{Capítulo 1}

\section{Introducción}

Un grafo es de comparabilidad si existe una orientación transitiva $\vec{E}$ de sus aristas. Un grafo de comparabilidad admite, en general, distintas orientaciones transitivas de sus aristas. Cada una de estas orientaciones define un poset: una relación de orden (reflexiva, antisimétrica y transitiva) $\leq_{\vec{E}}$ en $V_{G}$ de modo que

$$
u \leq_{\vec{E}} v \Leftrightarrow u=v \vee \overrightarrow{u v} \in \vec{E} .
$$

Dado un poset $P=(X, \leq)$, se dice que una familia de conjuntos $\mathcal{F}=\left(F_{i}\right)_{i \in I}$ es un modelo por contención de $P$ si existe una función $f: I \rightarrow X$ biyectiva tal que para todo $i$ y $j$ en $I$ se verifica que

$$
F_{i} \subset F_{j} \leftrightarrow f(i) \leq f(j) .
$$

Todo poset, y en consecuencia todo grafo de comparabilidad, puede ser modelado por contención. Distintas subclases de la clase de los grafos de comparabilidad han sido caracterizadas imponiendo condiciones geométricas a los conjuntos utilizados en los modelos por contención: ellos pueden ser intervalos de la recta real, regiones angulares en el plano, $d$-cajas en el espacio $\mathbb{R}^{n}, d$-esferas en el espacio $\mathbb{R}^{n}[7,13,23,25]$. En [13], 
se ha probado que cada grafo de comparabilidad admite un modelo por contención usando subárboles de una estrella (un árbol con un único vértice con grado mayor que uno).

Los grafos que admiten un modelo por contención de intervalos de la recta real, o grafos $C I$, han sido ampliamente estudiados y caracterizados de diferentes maneras. Uno de los primeros trabajos sobre esta clase de grafos fue el realizado por Dushnik y Miller [6], quienes demostraron que un grafo $G$ es $C I$ si y solamente si $G$ y su grafo complemento son grafos de comparabilidad.

Los grafos $C I$ han sido generalizados de diversas maneras. Una de esas generalizaciones es la clase de grafos que admiten un modelo por contención que asigna a los vértices caminos de un árbol, o brevemente modelo CPT (Containmen of Paths in a Tree).

La mayor parte de este trabajo está dedicado al estudio de aquellos grafos de comparabilidad y de aquellos posets que admiten un modelo CPT. Spinrad en [19] y Golumbic en [12] se preguntan sobre las propiedades de la clase de grafos $C P T$ y proponen el estudio de la misma.

Esta Tesis está organizada de la siguiente forma:

En el Capítulo 2, presentamos las definiciones básicas sobre grafos, posets y grafos de comparabilidad. También mostramos la relación existente entre grafos de comparabilidad, posets y modelos de contención.

En el Capítulo 3, caracterizamos a los vértices extremos de los grafos CI, es decir, aquellos vértices que pueden ocupar un extremo de una representación $C I$, usando una familia auto-complementaria de subgrafos inducidos prohibidos. Además, los grafos homogéneamente representables (todos sus vértices son vértices extremos) son caracterizados por una familia simple de subgrafos inducidos prohibidos.

En el Capítulo 4, presentamos una condición necesaria para que un poset sea CPT. A partir de ella describimos una familia de subposets prohibidos para la clase CPT. 
También mostramos ejemplos de posets que no son $C P T$ aun cuando satisfacen la condición necesaria. A continuación introducimos las definiciones de posets fuertemente$C P T$ y dualmente- $C P T$. En la última sección de este capítulo mostramos resultados relacionados con la dimensión de los posets $C P T$. Respondemos negativamente la pregunta sobre la dimensión de los grafos CPT que realizó Spinrad en [19].

En el Capítulo 5, demostramos que en la clase de los posets split la condición necesaria para ser un poset CPT dada en el Capítulo 4 es también suficiente. Como consecuencia obtenemos una caracterización por subposets prohibidos de los posets split CPT. Además mostramos que, en la clase de posets split, las clases de posets fuertemente- $C P T$ y dualmente- $C P T$ son coincidentes y las caracterizamos por subposets prohibidos. Por otra parte, caracterizamos por subgrafos inducidos prohibidos a la clase de grafos split fuertemente- $C P T$, la cual concide con la clase de grafos split dualmente- $C P T$.

En el Capítulo 6, estudiamos a los posets $k$-tree que admiten un modelo por contención de caminos en un árbol. Mostramos que en dicha clase la condición necesaria para ser poset $C P T$ es también suficiente. Obtenemos también una caracterización por subposets prohibidos de la clase de posets $k$-tree $C P T$, de las clases $k$-tree fuertemente- $C P T$ y $k$-tree dualmente- $C P T$ y presentamos una caracterización por subgrafos inducidos prohibidos de la clase de grafos $k$-tree fuertemente- $C P T$ y $k$-tree dualmente- $C P T$. Finalmente, en el Capítulo \%, damos algunas conclusiones. 


\section{Capítulo 2}

\section{Preliminares}

En este Capítulo presentamos las definiciones básicas, la notación y los resultados generales sobre grafos y conjuntos parcialmente ordenados que serán utilizados en el desarrollo de este trabajo. En general, las definiciones y la notación que adoptamos son consideradas estándar tanto en la Teoría de Órdenes como en la Teoría de Grafos. Como referencia se citan los libros de W. Trotter [23] y de J. Bondy y U. Murty [4].

\subsection{Grafos}

Un grafo simple $G$ es un par $(\mathrm{V}(\mathrm{G}), \mathrm{E}(\mathrm{G}))$, donde $V(G)$ es un conjunto finito no vacío, cuyos elementos se llaman vértices de $G$ y, $E(G)$ es un conjunto cuyos elementos son subconjuntos de dos elementos de $V(G)$. Los elementos de $E(G)$ se llaman aristas de $G$. Si $e=\{x, y\}$ es una arista, se notará $x y$, siendo $x$ e $y$ los vértices extremos de e. Una arista $e$ es incidente en un vértice $x$ si $x$ es un vértice extremo de $e$. El grado de un vértice es la cantidad de aristas incidentes en él. El vértice $x$ es adyacente a $y$ si $x y$ es una arista de $G$; en este caso también decimos que $x$ e $y$ son vecinos en $G$, y escribimos $x \sim y$. 
El vecindario abierto de un vértice $v$, o $N(v)$ es el conjunto de los vértices adyacentes a $v$. Es decir, $N(v)=\{x \in V(G): x v \in E(G)\}$. El vecindario cerrado del vértice $v$ se define por $N[v]=N(v) \cup\{v\}$. Un vértice $v$ es universal cuando $N[v]=V(G)$. Los vértices $x, y \in V(G)$ son gemelos si son adyacentes y $N(x)=N(y)$; y son falsos gemelos si no son adyacentes y $N(x)=N(y)$.

Diremos que dos grafos $G$ y $H$ son isomorfos si existe una biyección $f: V(G) \rightarrow$ $V(H)$ tal que $x y \in E(G)$ si y solo si $f(x) f(y) \in E(H)$.

Un grafo $H$ es un subgrafo de $G$ si $V(H) \subseteq V(G)$ y $E(H) \subseteq E(G)$. Si $V^{\prime}$ es un subconjunto no vacío de $V(G)$, entonces el subgrafo de $G$ inducido por $V^{\prime}$ es el subgrafo $H$ con $V(H)=V^{\prime}$ y $E(H)$ igual al conjunto de aristas de $G$ que tienen ambos extremos en $V^{\prime}$. El subgrafo inducido por $V^{\prime}$ es denotado por $G\left[V^{\prime}\right]$ y también solemos decir que $G$ tiene al grafo $G\left[V^{\prime}\right]$ como subgrafo inducido. Si $V^{\prime} \subseteq V(G)$, $G-V^{\prime}$ es el grafo que se obtiene quitando a $G$ los vértices pertenecientes a $V^{\prime}$ y las aristas incidentes en estos vértices. En otras palabras, $G-V^{\prime}$ es el subgrafo de $G$ inducido por el conjunto $V(G)-V^{\prime}$, es decir, $G-V^{\prime}=G\left[V(G)-V^{\prime}\right]$. Si $v \in V(G)$, denotaremos por $G-v$ al subgrafo inducido por $V(G)-\{v\}$.

Un camino de $G$ es una sucesión finita $\left[v_{0}, v_{1}, \ldots, v_{k}\right]$ de vértices de $G$ distintos entre sí tal que, para todo $i, 1 \leq i \leq k, v_{i-1} v_{i} \in E(G)$. Un vértices de grado uno en un camino se llama vértice extremo del camino. Se dice que $k$ es la longitud del camino y que éste conecta o une los vértices $v_{0}$ y $v_{k}$. Un camino cerrado o ciclo en $G$ es una sucesión finita $\left[v_{1}, \ldots, v_{k}, v_{k+1}\right]$ de vértices de $G$ con $v_{i} \neq v_{j}$ para $i \neq j$ e $i, j \in\{1, \ldots, k\}$ y $v_{1}=v_{k+1}$ tal que, para todo $i, 1 \leq i \leq k-1, v_{i-1} v_{i} \in E(G)$ y, además, $v_{k} v_{1} \in E(G)$. La longitud del ciclo es $k$. Una cuerda de un camino o en un ciclo es una arista entre dos vértices no consecutivos.

Un grafo es cordal si todo ciclo en $G$ con al menos cuatro vértices tiene una cuerda. Un grafo $G$ se dice conexo si para cualquier par de vértices distintos de $G$ existe un camino que los conecta. Una componente conexa de $G$ es un subgrafo conexo 
maximal de $G$. En este trabajo se usarán grafos conexos salvo que se diga lo contrario. Un árbol $T$ es un grafo conexo sin ciclos. Una hoja o vértice colgante de $T$ es un vértice de grado uno. Una arista de $T$ es pendiente si uno de sus extremos es una hoja de $T$. Una estrella es un árbol de $n$ vértices con un único vértice de grado mayor que uno, el cual recibe el nombre de centro de la estrella.

Un grafo completo de $n$ vértices tiene todos sus vértices adyacentes entre sí. Excepto isomorfismos, existe un único grafo completo con $n$ vértices. Tal grafo se denota por $K_{n}$. Si $G$ es un grafo, un completo de $G$ es un conjunto de vértices que induce un subgrafo completo. Un clique de $G$ es un completo maximal con respecto a la inclusión. Un conjunto de vértices no adyacentes de a pares es llamado conjunto estable.

El grafo complemento de $\mathbf{G}$, denotado por $\bar{G}$, es un grafo cuyo conjunto de vértices es $V(G)$ y tal que $x y \in E(\bar{G})$ si y solo si $x y \notin E(G)$. Observar que $K$ es un completo de $G$ si y solo si $K$ es un conjunto estable de $\bar{G}$.

Sea $\mathcal{F}$ una familia de grafos. Diremos que un grafo $G$ es $\mathcal{F}$-libre si ningún subgrafo inducido de $G$ es isomorfo a un miembro de la familia $\mathcal{F}$. Una clase de grafos $\mathcal{A}$ es $\mathcal{F}$-libre si todos los grafos perteneciente a la clase lo son. En este caso, los miembros de $\mathcal{F}$ se dicen subgrafos inducidos prohibidos para $\mathcal{A}$. Se dice que $H$ es subgrafo prohibido minimal para la clase $\mathcal{A}$ cuando $H$ es un subgrafo prohibido para $\mathcal{A}$ y $H-v \in \mathcal{A}$ para todo $v \in V(H)$.

Un grafo $G=(V, E)$ es un grafo de permutación si existen dos permutaciones $\sigma_{1}$, $\sigma_{2}$ sobre $V=\{1,2, \ldots, n\}$ tales que dos vértices $x, y$ de $V$ son adyacentes si y sólo si $\sigma_{1}(x)<\sigma_{1}(y)$ y $\sigma_{2}(y)<\sigma_{2}(x)$.

Un módulo (o conjunto homogéneo) en un grafo $G$ es un conjunto $M \subseteq V(G)$ tal que para todo $x, y \in M$ y $v \in V(G)-M, x v \in E(G)$ si y solamente si $y v \in E(G)$. En otras palabras, los vértices de $M$ son indistinguibles para cada vértice fuera de $M$. Los conjuntos $M=\{x\}$, para $x \in V(G), M=\emptyset$ y $M=V(G)$ son módulos de $G$ y se 
los llama módulos triviales de $G$. Se dice que $G$ es un grafo primo si sus únicos módulos son los triviales.

\subsection{Posets}

Un conjunto parcialmente ordenado o poset es un par $\mathbf{P}=(X, P)$ donde $X$ es un conjunto finito, llamado conjunto de elementos (o vértices), y $P$ es un orden parcial en $X$, es decir, una relación binaria reflexiva, antisimétrica y transitiva definida en $X$. Se escribe $x \leq y$ en $\mathbf{P}$ cuando $(x, y) \in P$; y $x<y$ en $\mathbf{P}$ cuando $(x, y) \in P$ y $x \neq y$. Si $x<y$ o $y<x$, decimos que $x$ e $y$ son comparables en $\mathbf{P}$ y lo denotamos $x \perp y$. Si $x$ e $y$ son dos elementos distintos que no son comparables en $\mathbf{P}$, diremos que son incomparables en $\mathbf{P}$ y notaremos $x \| y$. El elemento $x$ es cubierto por $y$ en $\mathbf{P}$, denotado por $x<: y$ en $\mathbf{P}$, cuando $x<y$ y no existe un elemento $z \in X$ para el cual $x<z$ y $z<y$.

Un poset $\mathbf{P}=(X, P)$ con una cantidad finita de elementos puede representarse mediante un diagrama de Hasse: a cada elemento de $X$ se le asigna un punto del plano de forma tal que si, $x<y$, el punto correspondiente a $x$ está por debajo del punto correspondiente a $y$. Luego, dos puntos se unen con una línea si $x<: y$.

Un conjunto $C \subseteq X$ es una cadena en un poset $\mathbf{P}$ si cualesquiera dos vértices de $C$ son comparables en $\mathbf{P}$. Sea $C$ una cadena de un poset $\mathbf{P}$ tal que no existe otra cadena en $\mathbf{P}$ que tenga a $C$ como subconjunto propio; entonces $C$ es una cadena maximal en $\mathbf{P}$. La altura de $\mathbf{P}$, que denotamos como $h(\mathbf{P})$, es el máximo entero $h$ para el cual existe una cadena de $h$ elementos en $\mathbf{P}$.

Si $z \in X$, su conjunto descendente en $\mathbf{P}$, denotado $D(z)$, y su conjunto ascendente en $\mathbf{P}$, denotado $U(z)$, son $\{x \in X: x<z\}$ y $\{x \in X: z<x\}$, respectivamente. Definimos $D[z]=D(z) \cup\{z\}$ y $U[z]=U(z) \cup\{z\}$. Usaremos la notación $D_{\mathbf{P}}(z)$ (o $\left.U_{\mathbf{P}}(z)\right)$ cuando sea necesario especificar el poset que estamos utilizando. 
Decimos que $z$ es un elemento minimal de $\mathbf{P}$ cuando $D(z)=\emptyset$. Cuando $U(z)=\emptyset$ decimos que $z$ es un elemento maximal de $\mathbf{P}$. Un elemento $x$ es máximo (mínimo) en $\mathbf{P}$ si $D[x]=X(U(x)=X)$. Dos elementos $x$ e $y$ del poset se dicen gemelos sin son comparables y $D(x) \cup U(x)=D(y) \cup U(y)$. Y se dicen falsos gemelos si son incomparables y $D(x) \cup U(x)=D(y) \cup U(y)$, lo cual ocurre si y solo si $D(x)=D(y)$ y $U(x)=U(y)$.

El dual de $\mathbf{P}$, denotado por $\mathbf{P}^{d}$, es el poset $\left(X, P^{d}\right)$ de modo que $P^{d}$ se define en la forma: $x<y$ en $\mathbf{P}^{d}$ si y solo si $y<x$ en $\mathbf{P}$.

La restricción de $P$ a un subconjunto $Y$ de $X$ se denota $P(Y)$. Llamaremos $\mathbf{P}(Y)$ al subposet $(Y, P(Y))$ de $\mathbf{P}$. Por simplicidad indicaremos $\mathbf{P}-Y$ para referirnos al subposet $\mathbf{P}(X-Y)$. Cuando $Y=\{y\}$, escribiremos $\mathbf{P}-y$ en lugar de $\mathbf{P}-\{y\}$.

Un orden parcial $P$ es un orden total (u orden lineal) si para todo $x, y \in X$ se verifica que $x \leq y$ en $\mathbf{P}$ o $y \leq x$ en $\mathbf{P}$. Sea $\mathbf{P}=(X, P)$ un poset; un orden lineal $L$ sobre $X$ es llamado extensión lineal de $P$ si $P \subseteq L$, es decir, si $x \leq y$ en $\mathbf{P}$ implica $x \leq y$ en $\mathbf{L}$. Es sabido que todo poset $\mathbf{P}=(P, X)$ tiene una extensión lineal [20]. Más aun, si $x \| y$ en $\mathbf{P}$ entonces existen dos extensiones lineales $L_{1}$ y $L_{2}$ tales que $x<y$ en $L_{1}$ e $y<x$ en $L_{2}$.

La dimensión de un poset $\mathbf{P}$, denotada por $\operatorname{dim}(\mathbf{P})$, es el menor entero positivo $k$ para el cual existen $L_{1}, L_{2}, \ldots, L_{k}$ extensiones lineales de $P$ tales que $P=\bigcap_{i=1}^{k} L_{i}$.

Un poset $\mathbf{P}=(X, P)$ se dice t-irreducible, para algún $t \geq 2$, si $\operatorname{dim}(\mathbf{P})=t$, y $\operatorname{dim}(\mathbf{P}(Y))<t$ para todo subconjunto propio $Y \subset X$.

Diremos que el poset $\mathbf{P}$ es conexo si para todo $x, y \in X$ con $x \neq y$ existe una secuencia finita $x=x_{0}, x_{1}, \ldots, x_{n}=y$ de elementos de $X$ tal que $x_{i} \perp x_{i+1}$ en $\mathbf{P}$ para $i=1,2, \ldots, n-1$. Salvo que se especifique lo contrario, siempre consideraremos posets conexos.

Los posets $\mathbf{P}=(X, P)$ y $\mathbf{Q}=(Y, Q)$ son isomorfos, y se escribe $\mathbf{P} \cong \mathbf{Q}$, si existe una biyección $f: X \rightarrow Y$ tal que $x_{1} \leq x_{2}$ en $\mathbf{P}$ si y solamente si $f\left(x_{1}\right) \leq f\left(x_{2}\right)$ en $\mathbf{Q}$. 
$\mathbf{A}_{\mathbf{0}}$

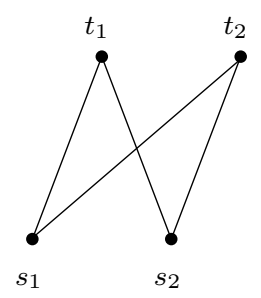

$\mathbf{A n}_{\mathbf{n}}$

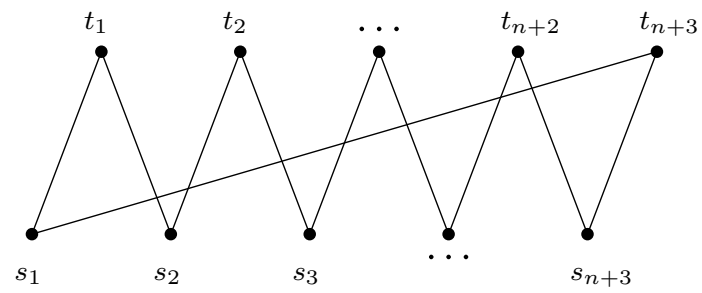

Figura 2.1: Diagrama de Hasse de la corona con cuatro elementos y de la corona con $2(\mathbf{n}+3)$ elementos, con $n \geq 0$.

Diremos que $f$ es un isomorfismo de $\mathbf{P}$ a $\mathbf{Q}$.

Un poset $\mathbf{P}=(X, P)$ es acíclico (o sin ciclos) si no tiene como subposets a ninguno de los poset descriptos en la Figura 2.1. Los posets de dicha figura son llamamos coronas. Observar que toda corona tiene al menos cuatro vértices.

Sea $\mathcal{F}$ una familia de posets. Diremos que un poset $\mathbf{P}$ es $\mathcal{F}$-libre si ningún subposet de $\mathbf{F}$ es isomorfo a un miembro de la familia $\mathcal{F}$. Una clase de posets $\mathcal{A}$ es $\mathcal{F}$-libre si todos los posets de la clase lo son. Como en el caso de los grafos, los miembros de la familia $\mathcal{F}$ se dicen subposets prohibidos para la clase $\mathcal{A}$. Y son prohibidos minimales si removiendo cualquiera de sus vértices se obtiene un poset perteneciente a la clase $\mathcal{A}$.

Resulta que los posets acíclicos son los posets corona-libre.

\subsection{Grafos de comparabilidad, posets y modelos por contención}

El grafo de comparabilidad $G_{\mathbf{P}}$ de un poset $\mathbf{P}$ es el grafo simple cuyo conjunto de vértices es $V\left(G_{\mathbf{P}}\right)=X$ y cuyo conjunto de aristas es $E\left(G_{\mathbf{P}}\right)=\{x y: x \perp y\}$. Diremos que dos posets son asociados si sus grafos de comparabilidad son isomorfos. En [22], Trotter et al. probaron el siguiente resultado que permite definir la dimensión de 
un grafo de comparabilidad $G$ como la dimensión de cualquier poset $\mathbf{P}$ tal que $G=G_{\mathbf{P}}$

Teorema 2.3.1. Si $\mathbf{P}$ y $\mathbf{P}^{\prime}$ son posets asociados entonces $\operatorname{dim}(\mathbf{P})=\operatorname{dim}\left(\mathbf{P}^{\prime}\right)$

Un grafo $G$ es un grafo de comparabilidad si existe algún poset $\mathbf{P}$ tal que $G=G_{\mathbf{P}}$. Una orientación $\vec{E}(G)$ (o simplemente $\vec{E}$ ) de $G$ es la asignación a cada arista $x y \in$ $E(G)$ de una de las dos direcciones posibles, $\overrightarrow{x y}$ o $\overrightarrow{y x}$. La orientación que a cada arista de $G$ le asigna la orientación opuesta a la dada por $\vec{E}$ se le dice orientación contraria a $\vec{E}$. Un vértice $v$ de $G$ es un sumidero (una fuente) en $\vec{E}$ si $\overrightarrow{x v} \in \vec{E}$ $(\overrightarrow{v x} \in \vec{E})$ para todo $x \in N(v)$.

Se dice que $\vec{E}$ es una orientación transitiva de $G$ si $\overrightarrow{x y} \in \vec{E}$ e $y z \in \vec{E}$ implican $\overrightarrow{x z} \in \vec{E}$. Es claro que si $\vec{E}$ es una orientación transitiva entonces su orientación contraria también lo es. Por otra parte, $v$ es un sumidero (fuente) en $\vec{E}$ si y solo si es fuente (sumidero) en la orientación contraria a $\vec{E}$.

Los grafos cuyas aristas pueden ser orientadas transitivamente son exactamente los grafos de comparabilidad [9].

Dada una orientación transitiva $\vec{E}$ de un grafo $G=(V, E)$, se denota $\mathbf{P}_{\vec{E}}$ al poset $\left(V, P_{\vec{E}}\right)$ donde $x<y$ en $\mathbf{P}_{\vec{E}}$ si y solo si $\overrightarrow{x y} \in \vec{E}$. Es claro que el grafo de comparabilidad de $\mathbf{P}_{\vec{E}}$ es $G$, y de esta manera, las orientaciones transitivas de $G$ están en una correspondencia biyectiva con los posets cuyos grafos de comparabilidad son isomorfos a $G$.

Sea $\vec{E}$ una orientación transitiva de $G=(V, E)$. Si $x y, x w \in E$ y $y w \notin E$, la orientación de una de las aristas fuerza la orientación de la otra. Es decir, $\overrightarrow{x y}, x \vec{w} \in \vec{E}$ o $\overrightarrow{y x}, \overrightarrow{w x} \in \vec{E}$. Como consecuencia de esta observación surge la definición de la relación $\Gamma$ definida sobre el conjunto de aristas de $G$ de la siguiente forma [11]: 


$$
x y \Gamma z w \Leftrightarrow \begin{cases}x z \notin E & \text { e } y=w \circ \\ y w \notin E & \text { у } x=z\end{cases}
$$

Esta relación binaria $\Gamma$ es reflexiva y simétrica, siendo su clausura transitiva una relación de equivalencia sobre las aristas de $G$. Las clases de esta relación se llaman clases de implicancia. Observar que si $G$ es un grafo de comparabilidad y $\vec{E}$ una orientación transitiva, la orientación de una de sus aristas nos permite conocer la orientación de las restantes aristas de la clase.

Diremos que un grafo de comparabilidad $G$ es UPO (Uniquely Partially Orderable) si $G=G_{\mathbf{P}} \cong G_{\mathbf{Q}}$ implica que $\mathbf{P} \cong \mathbf{Q}$ o $\mathbf{P} \cong \mathbf{Q}^{d}$.

Proposición 2.3.2. ([11]) Sea $G$ un grafo de comparabilidad. G es UPO si y solo si $G$ tiene una única clase de implicacia.

Gallai proporcionó la siguiente caracterización de los grafos de comparabilidad por una familia de subgrafos inducidos prohibidos minimales.

Teorema 2.3.3 ([8]). Un grafo es un grafo de comparabilidad si y solamente si ninguno de sus subgrafos inducidos es isomorfo a un grafo en la Figura $2.3 \mathrm{o}$ al complemento de un grafo en la Figura 2.4.

Un modelo por contención $M_{\mathbf{P}}$ de un poset $\mathbf{P}=(X, P)$ asigna a cada elemento $x \in X$ un conjunto $M_{x}$ de manera que $x<y$ en $\mathbf{P}$ si y solo si $M_{x}$ es subconjunto propio de $M_{y}$, es decir:

$$
x<y \operatorname{en} \mathbf{P} \Leftrightarrow M_{x} \subset M_{y} .
$$

Decimos que un modelo es inyectivo cuando dos elementos distintos no tienen asignado el mismo conjunto.

Notar que siempre podemos obtener un modelo por contención de un poset $\mathbf{P}=$ $(X, P)$ asignando a cada elemento $x$ su conjunto descendente cerrado $D[x]$. 

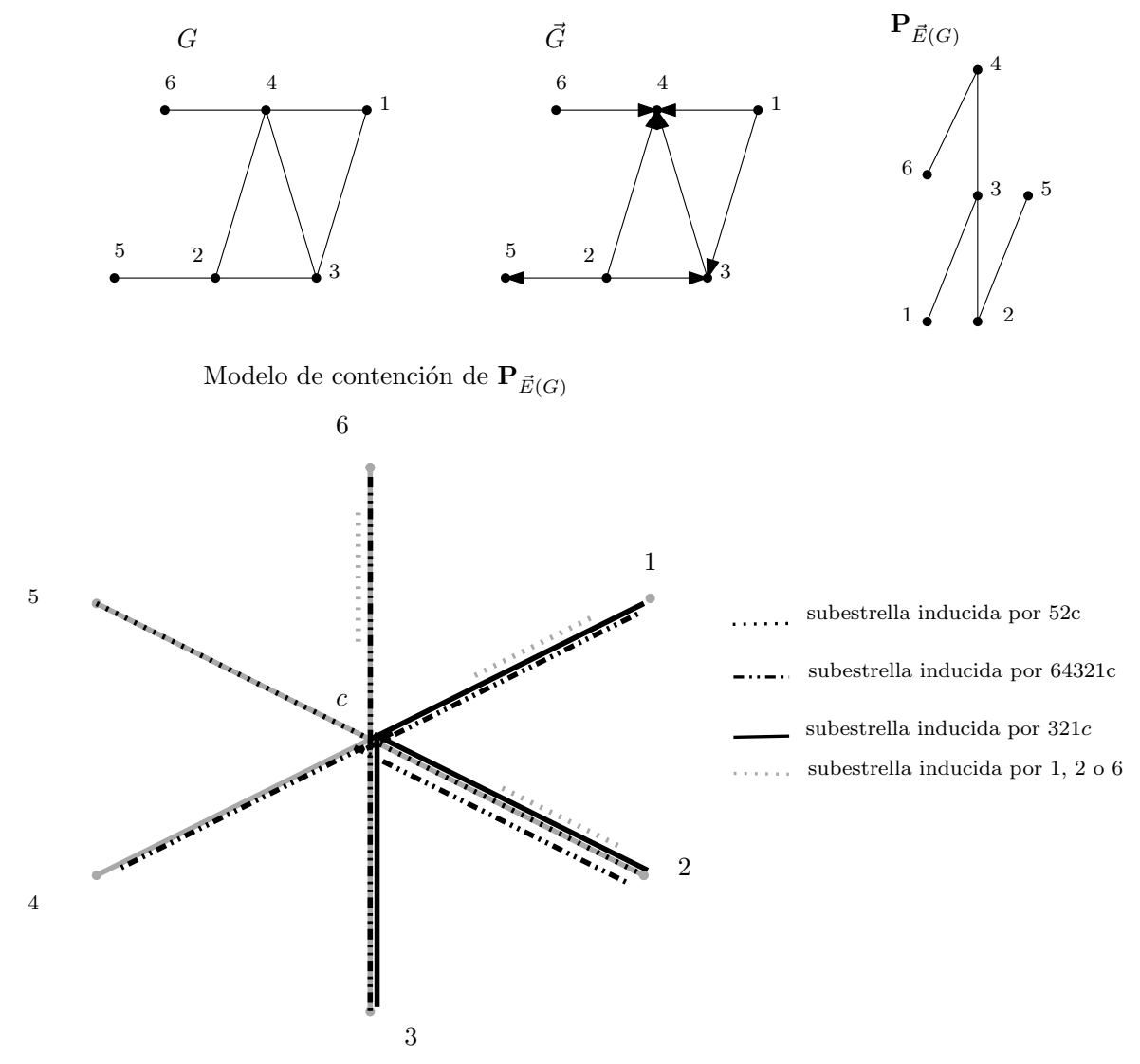

Figura 2.2: $G$ es un grafo de comparabilidad y $\mathbf{P}$ es un poset tal que $G=G_{\mathbf{P}}$. Entonces, $G$ admite un modelo $\left(M_{x}\right)_{x=1}^{6}$ por contención de subestrellas de una estrella tal que $x<y$ en $\mathbf{P} \Leftrightarrow \overrightarrow{x y} \in \vec{E}(G) \Leftrightarrow M_{x} \subset M_{y}$. 

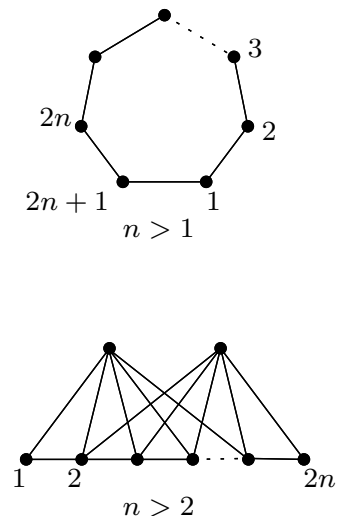
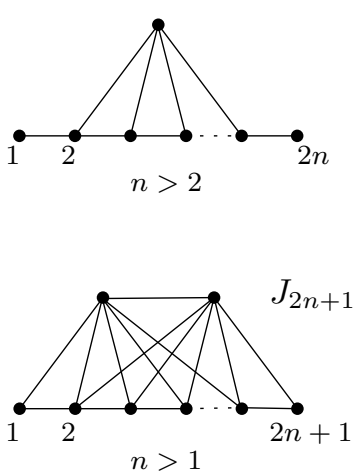

Figura 2.3: Estos grafos junto con los complementos de los grafos de la Figura 2.4 constituyen la familia de subgrafos inducidos prohibidos minimales para los grafos de comparabilidad

Un modelo por contención $M_{G}$ de un grafo $G$ asigna a cada vértice $v \in V(G)$ un conjunto $M_{v}$ de manera que $v$ es adyacente a $w$ en $G$ si y solo si $M_{v}$ es un subconjunto propio de $M_{w}$ o $M_{w}$ es subconjunto propio de $M_{v}$.

Observar que si $\left(M_{x}\right)_{x \in X}$ es un modelo por contención de un poset $\mathbf{P}$ entonces es un modelo por contención del grafo de comparabilidad $G_{\mathbf{P}}$, y más aun, el modelo determina una orientación transitiva $\vec{E}$ de $G_{\mathbf{P}}$ tal que $\mathbf{P}_{\vec{E}}=\mathbf{P}$.

Recíprocamente, un modelo por contención $\left(M_{v}\right)_{v \in V(G)}$ de un grafo $G$ determina una orientación transitiva $\vec{E}$ de $G$ y, consecuentemente, un poset $\mathbf{P}_{\vec{E}}$. Es claro que $\left(M_{v}\right)_{v \in V(G)}$ es también un modelo por contención de este poset.

Resulta que un grafo admite un modelo por contención si y solo si es de comparabilidad. Más aún, M. Golombic y E. Scheinerman demostraron en [13] que un grafo $G$ es de comparabilidad si y solo si $G$ admite un modelo por contención en el cual los conjuntos son subestrellas de una estrella.

En la Figura 2.2 podemos ver un el diagrama de Hasse de un poset junto con su grafo de comparabilidad orientado y un modelo por contención, donde a cada elemento $x \in\{1,2,3,4,5,6\}$ del poset se le asigna una subestrella de una estrella con seis hojas. 

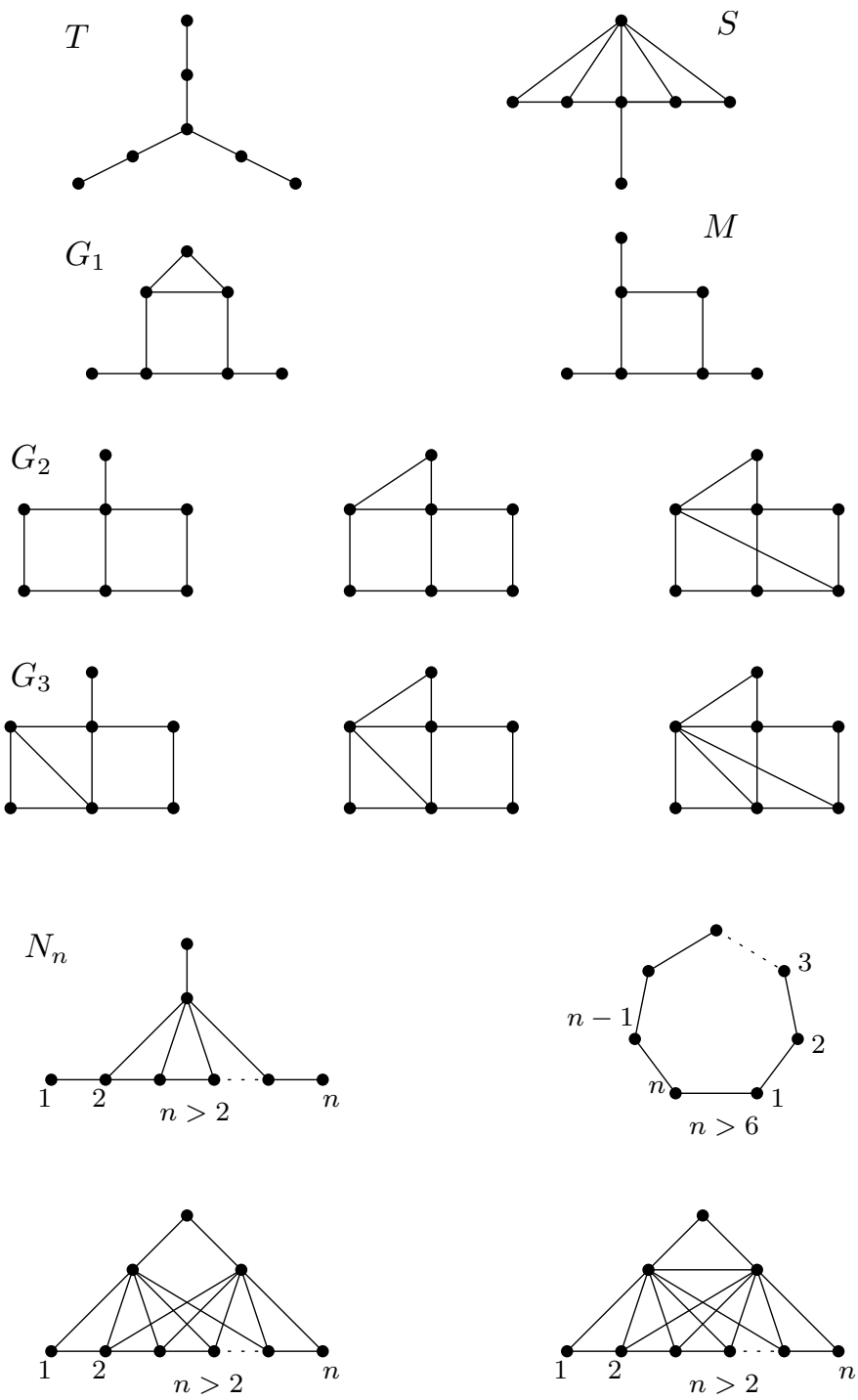

Figura 2.4: Los complementos de estos grafos juntos con los grafos de la Figura 2.3 constituyen la familia de subgrafos inducidos prohibidos minimales para los grafos de comparabilidad 
En [6], fue demostrado que $\operatorname{dim}(\mathbf{P}) \leq 2$ si y solamente si $\mathbf{P}$ admite un modelo por contención en el cual los conjuntos son intervalos de la recta real. Por lo tanto, los posets con dimensión a lo sumo 2 también aparecen en la literatura como ordenes de contención de intervalos, o simplemente $C I$ para abreviar. Los grafos de comparabilidad de los ordenes de contención de intervalos, o grafos CI, han sido caracterizados de diferentes maneras.

Teorema 2.3.4 ([6], [17]). Las siguientes afirmaciones son equivalentes.

1. G es un grafo $C I$.

2. Existe un poset $\mathbf{P}$ con $\operatorname{dim}(\mathbf{P}) \leq 2$ tal que $G=G_{\mathbf{P}}$.

3. $G$ y $\bar{G}$ son grafos de comparabilidad.

4. G es un grafo de permutación.

Una consecuencia del teorema anterior y del Teorema 2.3.1 es la siguiente.

Observación 2.3.5. Sean $\mathbf{P}$ y $\mathbf{P}^{\prime}$ posets asociados. Entonces, $\mathbf{P}$ es un poset $C I$ si $y$ solo si $\mathbf{P}^{\prime}$ es un poset $C I$. En particular, $\mathbf{P}$ es un poset $C I$ si y solo si $\mathbf{P}^{d}$ es un poset $C I$.

La caracterización de los posets de dimensión a lo sumo 2 mediante una familia de subposets prohibidos fue obtenida independientemente por Kelly y por Trotter-Moore.

Teorema 2.3.6 ([15] [21]). Un poset es CI si y solo sí ninguno de sus subposets es isomorfo a alguno de los posets descrito en las Figuras 2.5 y 2.6 ni a sus respectivos duales.

Es claro, como consecuencia del Teorema 2.3.4 y el hecho de que al remover un vértice cualquiera de un poset su dimensión disminuye en a lo sumo uno [23], que los subposets 

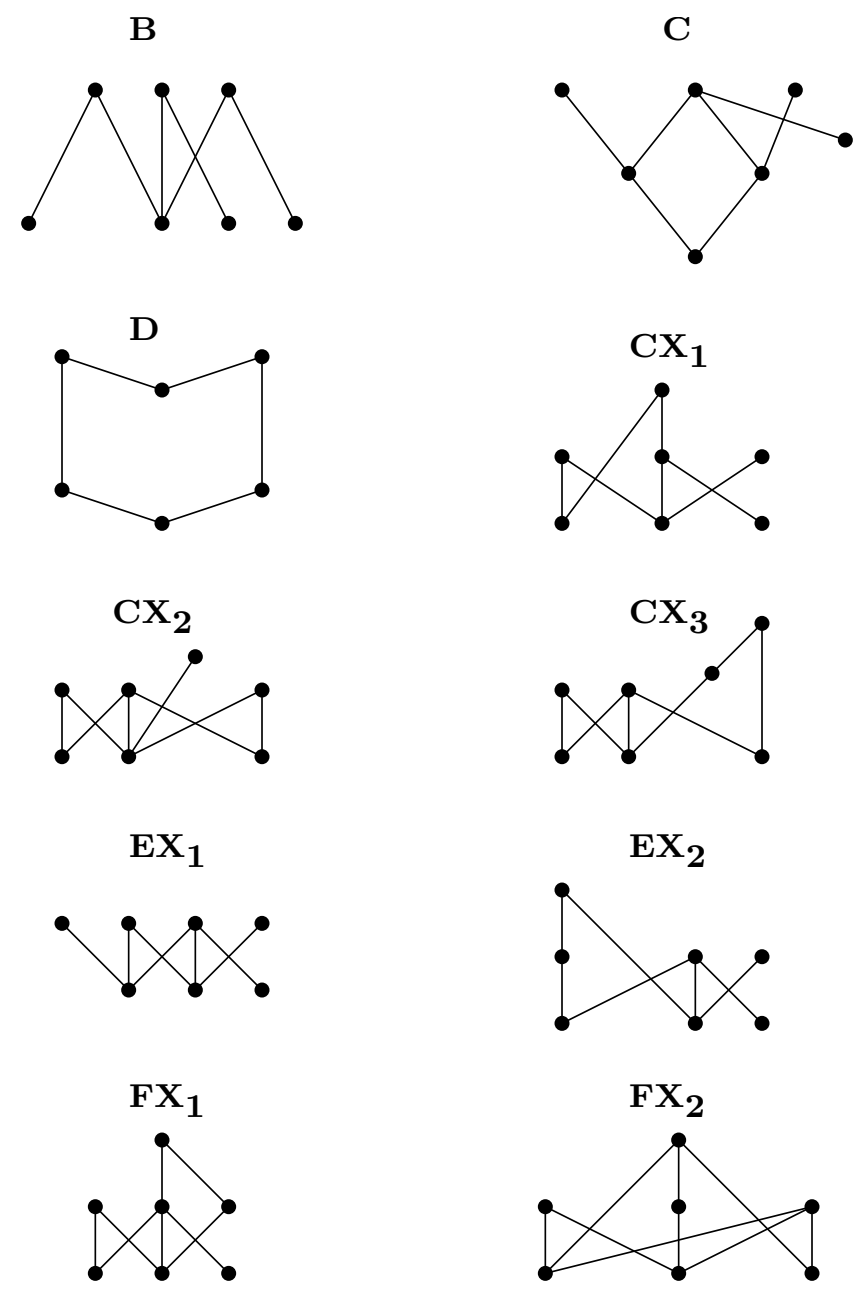

Figura 2.5: Estos posets y los posets de la Figura 2.6 junto con los correspondientes duales constituyen la familia de posets 3-irreducibles. 

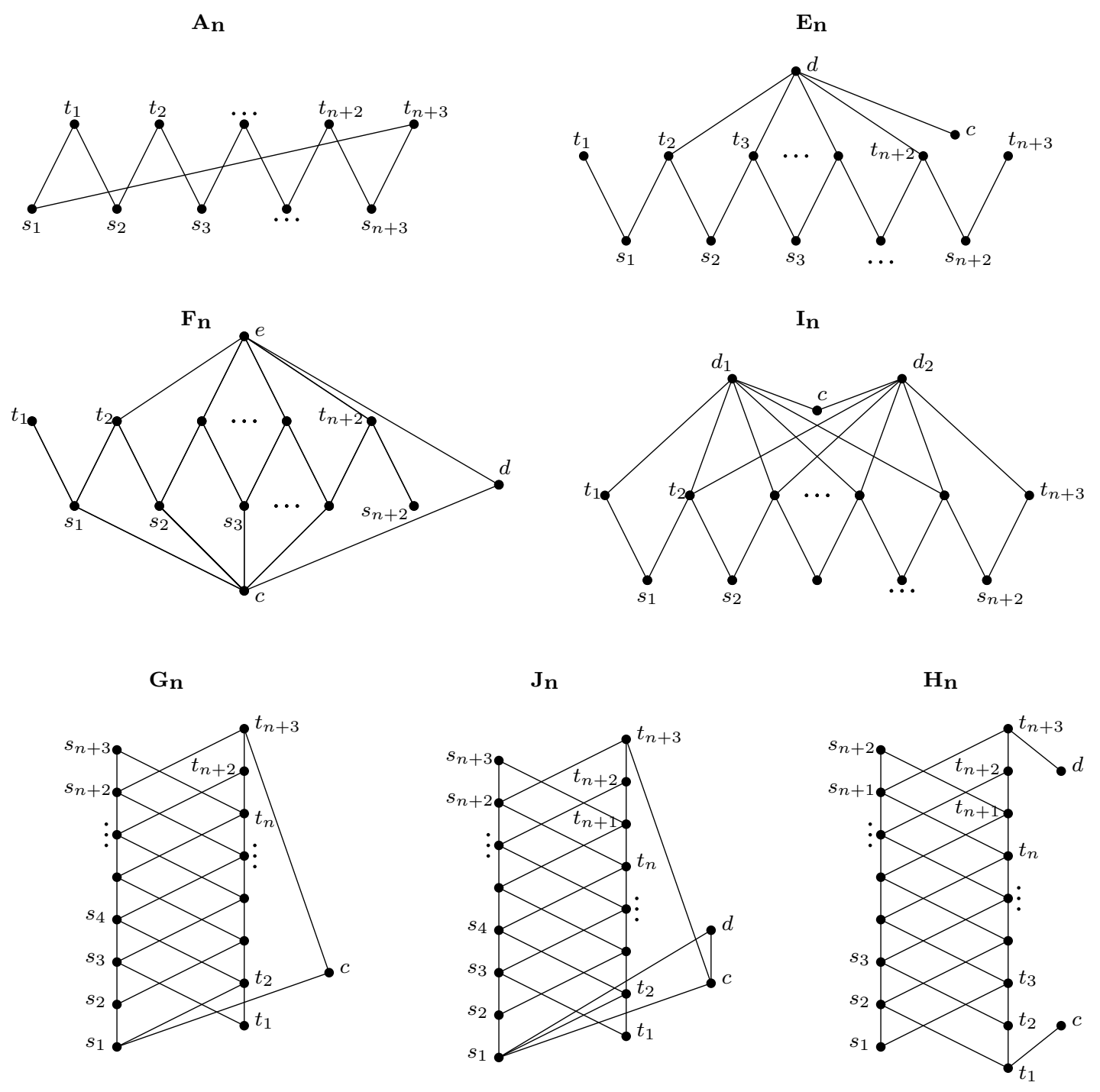

Figura 2.6: Estos posets y los posets de la Figura 2.5 junto con los correspondientes duales constituyen la familia de posets 3-irreducibles. 
prohibidos para posets $C I$ descritos en las Figuras 2.5 y 2.6 son exactamente los posets 3-irreducibles.

Un conjunto $M \subseteq X$ es un módulo de $\mathbf{P}=(X, P)$ si $M$ es módulo de $G_{\mathbf{P}}$. Entonces, para todo $x, y \in M$ y $v \in X-M$ se cumple que $x \perp v$ en $\mathbf{P}$ si y solo sí $y \perp v$ en $\mathbf{P}$. Más aun, $x<v$ en $\mathbf{P}$ si y solo sí $y<v$ en $\mathbf{P}$. Los módulos triviales de $G_{\mathbf{P}}, \emptyset, X$ y $\{x\}$ con $x \in X$, también son los módulos triviales de $\mathbf{P}$. Un poset se dice que es primo (o indescomponible) si todos sus módulos son triviales. En caso contrario, se dice que él es descomponible.

Proposición 2.3.7 ([23]). Sea $\mathbf{P}$ un poset $t$-irreducible para algún $t \geq 2$. Entonces $\mathbf{P}$ es primo.

El siguiente teorema dice que si un poset es primo su grafo de comparabilidad es $U P O$.

Teorema 2.3.8 ([8]). Sea $\mathbf{P}$ un poset primo. Si $\mathbf{P}^{\prime}$ es un poset asociado a $\mathbf{P}$ entonces $\mathbf{P}^{\prime}=\mathbf{P} o \mathbf{P}^{\prime}=\mathbf{P}^{d}$.

Corolario 2.3.9. Sea $\mathbf{S}$ un subposet de un poset $\mathbf{P}$ y sea $\mathbf{P}^{\prime}$ un poset asociado a $\mathbf{P}$. Se cumple que,

1. Si $\mathbf{P}$ es primo entonces $\mathbf{S}$ o $\mathbf{S}^{d}$ es un subposet de $\mathbf{P}^{\prime}$.

2. Si $\mathbf{S}$ es primo entonces $\mathbf{S}$ o $\mathbf{S}^{d}$ es un subposet de $\mathbf{P}^{\prime}$. 


\section{Capítulo 3}

\section{Vértices extremos de grafos $C I$}

En este Capítulo, describimos los vértices extremos de los grafos que admiten un modelo por contención de intervalos, es decir los grafos $C I$, usando una familia autocomplementaria de subgrafos inducidos prohibidos. Además, caracterizamos a los grafos $C I$ homogéneamente representables (todos sus vértices son vértices extremos) por subgrafos inducidos prohibidos.

\subsection{Caracterización de vértices extremos}

Recordemos que un grafo $G$ es un grafo $C I$ si existe una colección de intervalos $\left(I_{v}\right)_{v \in V(G)}$ de la recta real, a la cual llamaremos modelo o representación $C I$ de $G$ satisfaciendo que

$$
u v \in E \Leftrightarrow I_{u} \subset I_{v} \text { о } I_{v} \subset I_{u} .
$$

Sin pérdida de generalidad, se puede asumir que los intervalos de un modelo $C I$ son cerrados, tienen longitud positiva y dos intervalos distintos no tienen un extremo común [11, 19, 23]. 
En adelante llamaremos $l_{v}$ y $r_{v}$ a los extremos izquierdo y derecho del intervalo $I_{v}$, respectivamente. Un vértice $z \in V(G)$ se dice vértice extremo de la representación si $l_{z}<l_{v}$ para todo $v \in V-\{v\}$ o $r_{v}<r_{z}$ para todo $v \in V-\{v\}$.

Un vértice se dice vértice extremo de $G$ si es vértice extremo de alguna representación $C I$ de $G$; y se dice sumidero de $G$ (fuente de $G$ ) si es sumidero (fuente) en alguna orientación transitiva de $G$.

Observar que un vértice es una fuente de $G$ si y solo si es un sumidero de $G$.

Olariu y Gimbel, casi simultáneamente, obtuvieron una caracterización de los vértices sumideros en términos de subgrafos inducidos prohibidos, la cual citamos a continuación.

Teorema 3.1.1. ([10, 16]) Sea $G$ un grafo de comparabilidad. Un vértice $v$ de $G$ es un sumidero si y solamente si $G$ no contiene a ninguno de los siguientes grafos: $\mathbf{A}$, $\mathbf{B}, \mathbf{C}, \mathbf{D}_{\mathbf{2 k + 1}}$ con $k \geq 2, \overline{\mathbf{E}}_{\mathbf{n}}$ con $n \geq 3$, como subgrafo inducido con $v$ como vértice destacado ver Figura 3.1.

Cada representación $C I$ de $G$ induce naturalmente una orientación transitiva de las aristas: la arista $v w$ se orienta de $v$ hacia $w$ si $I_{v}$ es un subintervalo propio de $I_{w}$, y de $w$ hacia $v$ en caso contrario. Es claro que el intervalo correspondiente a un vértice extremo $z$ de la representación no puede estar contenido en otro. Luego toda arista incidente en $z$ estará orientada hacia $z$. Resulta que un vértice extremo de $G$ es necesariamente un sumidero de $G$.

Por otro lado, notemos que si $\left(I_{v}\right)_{v \in V}$ es un modelo $C I$ de $G$ con $I_{v}=\left[l_{v}, r_{v}\right]$ y $a$ es un punto de la recta a la derecha de todos estos intervalos, i.e., $r_{v}<a$ para todo $v \in V$, entonces los intervalos $I_{v}^{\prime}=\left[l_{v}, a+r_{v}\right]$ también constituyen un modelo $C I$ de $G$. Además, si invertimos el orden de los extremos derechos de estos intervalos considerando el intervalo $I_{v}^{\prime \prime}=\left[l_{v}, 2 a-r_{v}\right]$ (podemos suponer todos los extremos positivos) para cada vértice $v$, entonces $\left(I_{v}^{\prime \prime}\right)_{v \in V}$ es un modelo $C I$ del complemento 
de $G$.

Lo expuesto anteriormente prueba el siguiente lema:

Lema 3.1.2. Sea $G$ un grafo $C I$. Un vértice extremo de $G$ es necesariamente un sumidero de $G$ y de $\bar{G}$.

Veremos, como corolario del Teorema 3.1.4, que la implicación recíproca al lema anterior también es cierta. En la demostración de dicho teorema usaremos el lema a continuación.

Dado un modelo $C I$ de un grafo $G$, el conjunto derecho $R_{v}$ de un vértice $v$ de $G$ es $\left\{w \in V(G): l_{w}<r_{v}<r_{w}\right\}$; y el conjunto izquierdo $L_{v}$ es $\left\{w \in V(G): l_{w}<l_{v}<r_{w}\right\}$. Claramente, si $w \in R_{v} \cap L_{v}$ entonces $I_{v} \subset I_{w}$, y, consecuentemente, $w$ es adyacente a $v$.

Lema 3.1.3. Sea $\left(I_{v_{i}}\right)_{0 \leq i \leq k}$ un modelo $C I$ de un camino sin cuerdas $P=\left[v_{0} v_{1} \ldots v_{k}\right]$ con $k \geq 2$. Si $I_{v_{1}} \subset I_{v_{0}}$ entonces se cumple exactamente una de las siguientes afirmaciones:

(i) $v_{2} \in R_{v_{0}}$ y $r_{v_{0}}<r_{v_{i}}$ para $2 \leq i \leq k$.

(ii) $v_{2} \in L_{v_{0}}$ y $l_{v_{i}}<l_{v_{0}}$ para $2 \leq i \leq k$.

Demostración: Procederemos por inducción sobre $k$. La proposición es obviamente cierta cuando $k=2$. Sea $k>2$ y asumamos que $I_{v_{1}} \subset I_{v_{0}}$. Como $v_{2}$ es adyacente a $v_{1}$ y no es adyacente a $v_{0}$, tenemos que $v_{2} \in R_{v_{0}}-L_{v_{0}}$ o $v_{2} \in L_{v_{0}}-R_{v_{0}}$. Asumamos, sin pérdida de generalidad, que $v_{2} \in R_{v_{0}}$. Por hipótesis inductiva resulta $r_{v_{0}}<r_{v_{i}}$ para $2 \leq i \leq k-1$. Para completar la demostración, mostraremos que $r_{v_{0}}<r_{v_{k}}$.

Dado que $v_{k}$ es adyacente a $v_{k-1}$, se tiene que $I_{v_{k-1}} \subset I_{v_{k}}$ o $I_{v_{k}} \subset I_{v_{k-1}}$. En el primer caso, es claro que $r_{v_{0}}<r_{v_{k-1}}<r_{v_{k}}$. Por lo tanto, asumamos que $I_{v_{k}} \subset I_{v_{k-1}}$; y, con el objetivo de llegar a una contradicción, supongamos que $r_{v_{k}}<r_{v_{0}}$. 
A

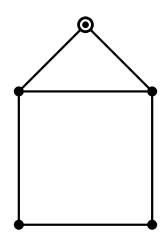

B

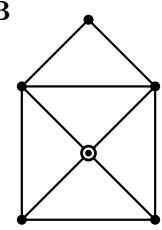

C

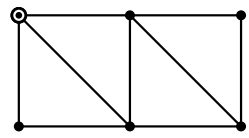

$\mathbf{D}_{n}$

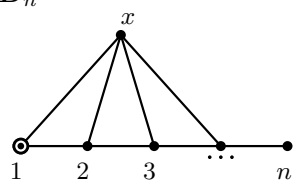

$\mathbf{E}_{n}$

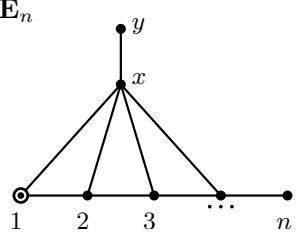

$\overline{\mathbf{A}}$

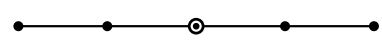

$\overline{\mathbf{B}}$

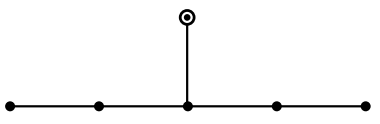

$\overline{\mathbf{C}}$

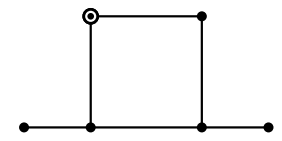

$\overline{\mathbf{D}}_{n}$

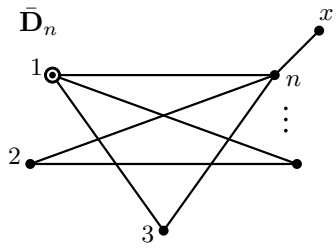

$\overline{\mathbf{E}}_{n}$

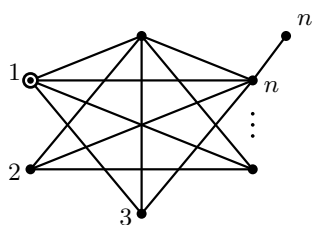

Figura 3.1: Cada vértice destacado en los grafos $\mathbf{A}, \mathbf{B}, \mathbf{C}, \mathbf{D}_{\mathbf{2 k}+\mathbf{1}}$ con $k \geq 2, \overline{\mathbf{E}}_{\mathbf{n}}$ con $k \geq 3$, no puede ser sumidero de un grafo de comparabilidad. Cada vértice destacado en los grafos $\mathbf{A}, \overline{\mathbf{A}}, \mathbf{B}, \overline{\mathbf{B}}, \mathbf{C}, \overline{\mathbf{C}}, \mathbf{D}_{\mathbf{2 k + 1}}, \overline{\mathbf{D}}_{\mathbf{2 k + 1}}, \mathbf{E}_{\mathbf{3}}, \mathbf{E}_{\mathbf{2 k}}, \overline{\mathbf{E}}_{\mathbf{2 k}}$, para con $k \geq 2$, no puede ser vértice extremo de un grafo $C I$. 
Debido a que $r_{v_{0}}<r_{v_{k-1}}$ y $v_{k-1}$ no es adyacente a $v_{0}$, obtenemos que $l_{v_{0}}<l_{v_{k-1}}$. Por lo tanto, $l_{v_{0}}<l_{v_{k-1}}<l_{v_{k}}$, y entonces $I_{v_{k}} \subset I_{v_{0}}$, lo cual contradice el hecho de que $v_{k}$ y $v_{0}$ no son adyacentes.

Teorema 3.1.4. Sea $G=(V, E)$ un grafo $C I$. Un vértice $z$ es un vértice extremo de $G$ si y solo si $G$ no contiene a ninguno de los grafos $\mathbf{A}, \overline{\mathbf{A}}, \mathbf{B}, \overline{\mathbf{B}}, \mathbf{C}, \overline{\mathbf{C}}, \mathbf{D}_{\mathbf{2 k + 1}}, \overline{\mathbf{D}}_{\mathbf{2 k + 1}}$, $\mathbf{E}_{\mathbf{3}}, \mathbf{E}_{\mathbf{2}}, \overline{\mathbf{E}}_{\mathbf{2}}$, para $k \geq 2$, como subgrafo inducido, con z como vértice destacado de la Figura 3.1.

Demostración: La implicación directa es consecuencia del Lema 3.1.2 y del Teorema 3.1.1. Observar que $\overline{\mathbf{E}}_{\mathbf{3}}=\mathbf{E}_{\mathbf{3}}$ y que $\mathbf{D}_{\mathbf{n}}$ es subgrafo inducido de $\mathbf{E}_{\mathbf{n}}$.

Para demostrar la proposición recíproca, consideremos el grafo $G^{\prime}$ que se obtiene agregando a $G$ un vértice pendiente $z^{\prime}$ adyacente a $z$.

Observar primero que, por el Teorema 3.1.1, el vértice $z$ es un sumidero de $G$. Luego existe una orientación transitiva $\vec{E}$ de $G$ en la cual todas las aristas incidentes en $z$ están orientadas hacia $z$. La orientación transitiva $\overrightarrow{E^{\prime}}$ de $G^{\prime}$ que se obtiene agregando $\overrightarrow{z^{\prime}} z$ a $\vec{E}$ prueba que $G^{\prime}$ es de comparabilidad y que $z$ es un sumidero de $G^{\prime}$. Probaremos a continuación que $G^{\prime}$ es $C I$.

Asumamos, con el objetivo de obtener una contradicción, que $G^{\prime}$ no es $C I$. Luego, $G^{\prime}$ contiene un subgrafo inducido $H$ el cual es un grafo en la Figura 2.4, o bien el complemento de un grafo de la Figura 2.3; más aun, $H$ debe contener a $z^{\prime}$, por lo que $H$ debe tener un vértice de grado uno y $z$ es el único vecino de ese vértice.

Una inspección de esas figuras revela que $H$ tiene que ser uno de los grafos $T, S, M$, $G_{1}, G_{2}, G_{3}$ o $N_{n}$ para algún $n \geq 4$, en la Figura 2.4; o el complemento del grafo $J_{2 k+1}$ para algún $k \geq 2$ en la Figura 2.3. En el siguiente párrafo mostraremos que cada caso implica una contradicción, y que por lo tanto $G^{\prime}$ es un grafo $C I$.

- Si $H$ es alguno de los grafos $S, G_{1}, G_{2}$ o $G_{3}$, entonces $G$ contiene al grafo $\overline{\mathbf{A}}$ en 
la Figura 3.1 con z como el vértice señalado.

- Si $H$ es el grafo $T$, entonces $G$ contiene el grafo $\overline{\mathbf{B}}$ en la Figura 3.1 con $z$ como el vértice señalado.

- Si $H$ es el grafo $M$, entonces tenemos que considerar dos casos dependiendo de cual vértice de grado uno es $z^{\prime}$ : su vecino $z$ es adyacente a dos vértices con grado 3 , o su vecino $z$ es adyacente a un vértice con grado 3 y otro con grado 2. En el primer caso, $G$ contiene el grafo $\overline{\mathbf{A}}$ en la Figura 3.1 con z como vértice marcado; y, en el último, $G$ contiene al grafo $\overline{\mathbf{C}}$ en Figura 3.1 con $z$ como el vértice señalado.

- Si $H$ es el grafo $N_{4}$, entonces $G$ contiene al grafo $\mathbf{E}_{\mathbf{3}}$ en Figura 3.1 con $z$ como el vértice marcado.

- Si $H$ es el grafo $N_{n}$ para algún $n>4$, entonces, nuevamente, consideramos dos casos dependiendo de cuál vértice de grado uno es $z^{\prime}$. Si $z^{\prime}$ es el vértice etiquetado 1 (o $n$ ) entonces $G$ contiene el grafo $\mathbf{E}_{\mathbf{n}-\mathbf{1}}$ y $\mathbf{D}_{\mathbf{n}-\mathbf{1}}$ en Figura 3.1 con $z$ como el vértice señalado. Notar que $\mathbf{E}_{\mathbf{n}-\mathbf{1}}$ es prohibido cuando $n$ es impar y $\mathbf{D}_{\mathbf{n}-1}$ es prohibido cuando $n$ es par. Si $z^{\prime}$ no es el vértice etiquetado 1 ni el vértice etiquetado $n$, entonces $G$ contiene el grafo $\overline{\mathbf{A}}$ en la Figura 3.1 con $z$ como el vértice señalado.

Por lo tanto, hemos probado que $G^{\prime}$ es $C I$.

Sea $\left(I_{w}\right)_{w \in V\left(G^{\prime}\right)}$ un modelo $C I$ de $G^{\prime}$ compatible con $\overrightarrow{E^{\prime}}$, es decir,

$$
\overrightarrow{u v} \in \overrightarrow{E^{\prime}} \text { implica } I_{u} \subset I_{v} \text {. }
$$

Claramente, si $I_{z}$ es un intervalo extremo de esta representación entonces $z$ es un vértice extremo de $G$ y el teorema está probado. Asumamos entonces que z no es 
extremo en la representación $\left(I_{w}\right)_{w \in V\left(G^{\prime}\right)}$. Resulta que el conjunto izquierdo $L_{z}$ y el conjunto derecho $R_{z}$ de $z$ son no vacíos. El hecho que $z$ es el único vecino de $z^{\prime}$ implica que $L_{z} \cap R_{z}=\emptyset$.

Sea $x$ un vértice de $L_{z}$ minimizando la distancia a $z$ en $G^{\prime}$. Sea $P_{z x}=\left[v_{0}=z, v_{1}, v_{2}, \ldots, v_{k}=x\right]$ un camino de mínima longitud uniendo $z$ con $x$. Como $z$ es un sumidero, tenemos que $\overrightarrow{v_{1} z} \in \overrightarrow{E^{\prime}}$, lo cual implica que $I_{v_{1}} \subset I_{z}$. Resulta, por el Lema 3.1.3, que $v_{2} \in R_{z} \mathrm{y}$ $r_{z}<r_{v_{i}}$ para todo $i$, o $v_{2} \in L_{z}$ y $l_{v_{i}}<l_{z}$ para todo $i$ mayor que 0 .

Como $v_{k}=x \in L_{z}$ debe ser $v_{2} \in L_{z}$ y $l_{v_{i}}<l_{z}$ para todo $i$. Como $x$ fue elegido minimizando la distancia a $z$ debe ser $v_{2}=v_{k}=x$ y así probamos la existencia de un camino de la forma $\left[z, v_{1}, x\right]$. Análogamente, existen los vértices $v_{1}^{\prime}$, e $y \in R_{z}$ induciendo el camino $\left[z, v_{1}^{\prime}, y\right]$.

La prueba será completada mostrando que la concatenación de ambos caminos induce el grafo $\overline{\mathbf{A}}$ con $z$ como el vértice marcado.

Claramente, $x$ e $y$ no son adyacentes. Afirmamos que $x$ no es adyacente a $v_{1}^{\prime}$. De hecho, si $x$ fuera adyacente a $v_{1}^{\prime}$, se obtiene que $r_{v_{1}^{\prime}}<r_{x}$; por otro lado, ya que $z^{\prime}$ tiene grado uno e $I_{z^{\prime}} \subset I_{z}$, se sigue que $r_{x}<r_{z^{\prime}}$ y $l_{z^{\prime}}<l_{y}$. Por lo tanto,

$$
l_{z^{\prime}}<l_{y}<l_{v_{1}^{\prime}}<r_{v_{1}^{\prime}}<r_{x}<r_{z^{\prime}}
$$

lo cual implica $I_{v_{1}^{\prime}} \subset I_{z^{\prime}}$, contrario al hecho de que $z^{\prime}$ es adyacente únicamente a $z$. Análogamente, $y$ no es adyacente a $v_{1}$.

Como consecuencia de los Teoremas 3.1.1 y 3.1.4, obtenemos el siguiente resultado.

Teorema 3.1.5. Sea $G$ un grafo $C I$. Un vértice $z$ es un vértice extremo de $G$ si $y$ solo sí $z$ es un sumidero de $G$ y $\bar{G}$. 


\subsection{Grafos $C I$ homogéneamente representables}

Un grafo $C I G$ es homogéneamente representable si cada uno de sus vértices es un vértice extremo.

Teorema 3.2.1. Sea $G$ un grafo $C I$. Entonces $G$ es homogéneamente representable si y solo si no contiene a los grafos $\mathbf{A}, \overline{\mathbf{A}}, \mathbf{C}, \overline{\mathbf{C}}, \mathbf{E}_{\mathbf{3}}$, descritos en la Figura 3.1, como subgrafos inducido.

Demostración: Es una consecuencia directa del Teorema 3.1.4 y el hecho que: A es un subgrafo inducido de $\mathbf{B}$ y de $\mathbf{D}_{\mathbf{2 k + 1}}$, para $k \geq 2 ;$ y $\mathbf{E}_{\mathbf{3}}$ es un subgrafo inducido de $\mathbf{E}_{2 \mathbf{k}}$, para $k \geq 2$.

En el teorema previo, la condición ' $G$ es un grafo $C I$ ' puede ser relajada agregando $C_{5}$ (el ciclo sin cuerdas con 5 vértices) a la familia de subgrafos prohibidos.

Teorema 3.2.2. Un grafo $G$ es $C I$ homogéneamente representable si y solo sí $G$ no contiene a los grafos $C_{5}, \mathbf{A}, \overline{\mathbf{A}}, \mathbf{C}, \overline{\mathbf{C}}, \mathbf{E}_{\mathbf{3}}$, descripto en la Figura 3.1, como subgrafos inducido.

Demostración: Si cada vértice de $G$ es extremo entonces, por el Teorema 3.1.4, $G$ no contiene a ninguno de los grafos de la Figura 3.1; en particular no contiene a $\mathbf{A}$, $\overline{\mathbf{A}}, \mathbf{C}, \overline{\mathbf{C}}$ ni $\mathbf{E}_{\mathbf{3}}$. Además, $C_{5}$ es un prohibido de los grafos de comparabilidad, por lo que $G$ no lo contiene.

Recíprocamente sea $G$ un grafo que no contiene a $C_{5}, \mathbf{A}, \overline{\mathbf{A}}, \mathbf{C}, \overline{\mathbf{C}}$ ni $\mathbf{E}_{\mathbf{3}}$ como subgrafos inducidos. Recordemos que un grafo cualquiera $H$ es $C I$ si y solo si ninguno de los grafos de las Figuras 2.3 y 2.4 ni los complementos de dichos grafos es subgrafo inducido de $H$. Cada uno de estos prohibidos tiene como subgrafo inducido a $C_{5}, \mathbf{A}$, $\overline{\mathbf{A}}, \mathbf{C}, \overline{\mathbf{C}}$ o $\mathbf{E}_{\mathbf{3}}$. Por lo tanto, podemos asegurar que $G$ es $C I$; y por el Teorema 3.2.1 él es homogéneamente representable. 


\section{Capítulo 4}

\section{Posets CPT: Propiedades Generales y Dimensión}

En la Primera sección de este Capítulo, presentamos una condición necesaria para que un poset sea $C P T$. A partir de ella describimos una familia de subposets prohibidos para la clase CPT. También mostramos diferentes ejemplos de posets que no son CPT aún cuando satisfacen la condición necesaria. Introducimos las definiciones de posets fuertemente- $C P T$ y dualmente- $C P T$. En la tercera sección, demostramos que en la clase de posets $C P T$ la dimensión y la dimensión de intervalos no están acotadas superiormente. Sin embargo, la dimensión de un poset $C P T \mathbf{P}$ cualquiera está acotada superiormente por la cantidad de hojas del árbol huésped de cualquier modelo $C P T$ de $\mathbf{P}$.

\subsection{Propiedades Generales}

Definición 4.1.1. Un poset $\mathbf{P}=(X, P)$ es un orden de contención de caminos en un árbol o poset $\boldsymbol{C P T}$ si admite un modelo por contención $M_{\mathbf{P}}=\left(W_{x}\right)_{x \in X}$ tal que cada $W_{x}$ es el conjunto de vértices de un camino de un árbol. 
Modelo CPT de $\mathbf{B}$

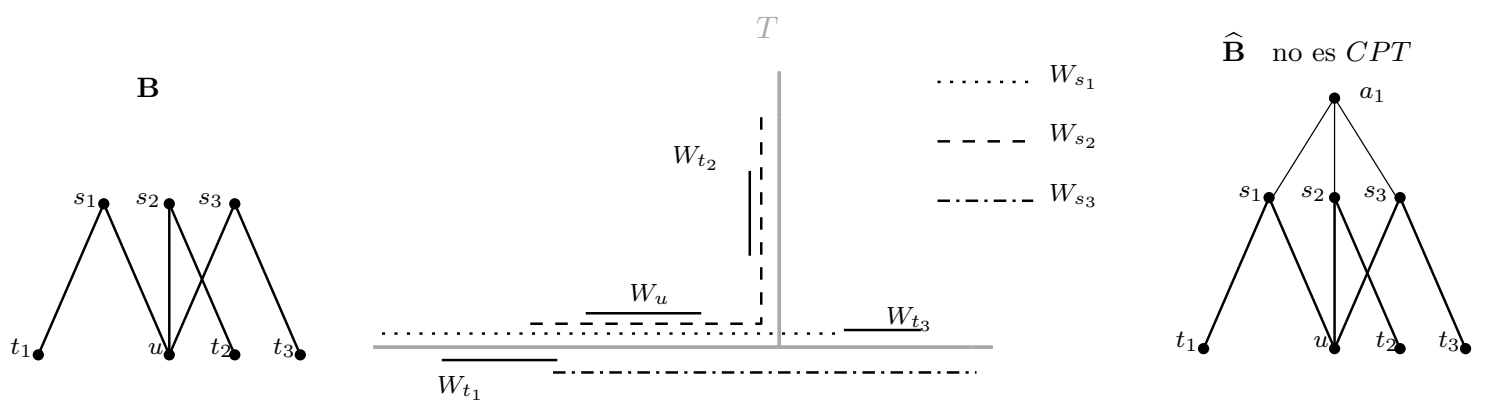

Figura 4.1: El poset $\mathbf{B}$ admite un modelo $C P T$, pero el poset $\widehat{\mathbf{B}}$ no es $C P T$.

El árbol utilizado como soporte de los caminos se llama árbol huésped del modelo. Con el objetivo de simplificar la notación, de ahora en adelante identificaremos los caminos con sus conjuntos de vértices. Es decir, también nos referiremos a los conjuntos $W_{x}$ como si fuesen caminos. Consecuentemente, podremos decir que un camino $W$ está contenido en un camino $W^{\prime}$ o que un camino $W$ es un subconjunto propio de un camino $W^{\prime}$; y escribir, por ejemplo, sean $W$ y $W^{\prime}$ caminos tales que $W \subseteq W^{\prime}$ o tales que $W \subset W^{\prime}$.

En la Figura 4.1 podemos ver el diagrama de Hasse del poset 3-irreducible $\mathbf{B}=$ $(X, B)$ donde $X=\left\{s_{1}, s_{2}, s_{3}, t_{1}, t_{2}, t_{3}\right\}$, junto con un modelo $C P T$ del mismo. A cada elemento del conjunto $X$ se le asigna un camino en el árbol $T$ de forma que un elemento es menor que otro en el poset $\mathbf{B}$ si y solo si el camino correspondiente al primer elemento es un subconjunto (subcamino) propio del camino correspondiente al segundo.

A modo de ejemplo, observar que $W_{t_{1}} \subset W_{s_{1}}$, pero $W_{t_{1}} \not \subset W_{s_{2}}$, y $W_{t_{1}} \not \subset W_{s_{3}}$, ya que $t_{1}<s_{1}, t_{1} \| s_{2}$ y $t_{1} \| s_{3}$ en $\mathbf{B}$.

El poset $\widehat{\mathbf{B}}$, cuyo diagrama de Hasse podemos ver en la misma figura, no admite un modelo $C P T$. En efecto, si $\widehat{\mathbf{B}}$ admitiese un modelo $C P T\left(P_{x}\right)_{x \in X \cup\left\{a_{1}\right\}}$, entonces tendríamos $P_{x} \subset P_{a_{1}}$ para cada elemento $x \in D\left(a_{1}\right)=X$. Luego, como $P_{a_{1}}$ es un camino, claramente tendríamos un modelo $C I$ de $\mathbf{B}$, lo cual contradice el hecho de 
que $\mathbf{B}$ no es un poset $C I$ (ver Teorema 2.3.6 y la Figura 2.5).

En el siguiente lema mostramos una condición necesaria para que un poset sea $C P T$.

Lema 4.1.2. Si $\mathbf{P}$ es un poset CPT entonces para todo elemento z de $\mathbf{P}$ el subposet inducido por el conjunto descendente cerrado de z $\mathbf{P}(D[z])$ es un poset $C I$.

Demostración: Sea $\left(W_{x}\right)_{x \in X}$ un modelo $C P T$ de $\mathbf{P}$. Como $x \in D(z)$ implica $W_{x} \subset$ $W_{z}$, tenemos que cada $W_{x}$ con $x \in D[z]$ es un subintervalo de $W_{z}$. Por lo tanto, $\left(W_{x}\right)_{x \in D[z]}$ es un modelo por contención de intervalos de $\mathbf{P}(D[z])$.

Recordemos que un elemento máximo en un poset es un elemento mayor que todo otro elemento del poset y que los posets 3-irreducibles descriptos en las Figuras 2.5 y 2.6 caracterizan por subposets prohibidos a los posets $C I$.

Definición 4.1.3. Dado un poset $\mathbf{P}$, indicaremos mediante $\widehat{\mathbf{P}}$ al poset que se obtiene agregando a $\mathbf{P}$ un elemento máximo. Llamaremos $\boldsymbol{T o p}_{3-i r r e d}$ al conjunto $\{\widehat{\mathbf{P}}: \mathbf{P}$ poset 3-irreducible $\}$.

Una consecuencia del Lema 4.1.2 es que cada poset en la clase $\mathbf{T o p}_{\mathbf{3} \text {-irred }}$ es un ejemplo de poset que no es CPT. En lo que sigue mostraremos cuáles de ellos son minimales.

Lema 4.1.4. Todo poset de la clase $\boldsymbol{T o p}_{3-\text { irred }}$ distinto de $\widehat{\mathbf{I}_{\mathbf{n}}}$, para todo $n \geq 0$, es un poset prohibido minimal para la clase CPT.

\section{Demostración:}

Sea $\mathbf{P}=(X, P)$ un poset 3-irreducible (ver Figuras 2.5 y 2.6) y llamemos $u$ al elemento máximo de $\widehat{\mathbf{P}}$. Por el Lema 4.1 .2 , el poset $\widehat{\mathbf{P}}$ no es $C P T$. Veamos que es minimal. Sea $x$ es un elemento de $X$. Un modelo $C P T$ de $\widehat{\mathbf{P}}-x$ se obtiene considerando un modelo $C I$ de $\mathbf{P}-x$ y agregando un camino $W_{u}$ correspondiente al vértice máximo $u$ que contenga todos los caminos del modelo $C I$.

Veremos a continuación que $\widehat{\mathbf{P}}-u=\mathbf{P}$ es $C P T$ si $\mathbf{P} \neq \mathbf{I}_{\mathbf{n}}$. 
Primero, consideremos el caso en que $\mathbf{P}$ tiene un elemento maximal $x$ tal que su conjunto descendente es una cadena. Observar que en tal caso $\mathbf{P}$ debe ser alguno de los siguientes posets: $\mathbf{B}^{d}, \mathbf{C}, \mathbf{C}^{d}, \mathbf{C X}_{1}, \mathbf{C X}_{1}^{d}, \mathbf{C X}_{2}, \mathbf{E X}_{1}, \mathbf{E X}_{1}^{d}, \mathbf{E X}_{\mathbf{2}} \cong \mathbf{E X}_{2}^{d}, \mathbf{F X}_{1}^{d}$, $\mathbf{E}_{\mathbf{n}}, \mathbf{E}_{\mathbf{n}}^{d}, \mathbf{F}_{\mathbf{n}} \cong \mathbf{F}_{\mathbf{n}}^{d}$ o $\mathbf{H}_{\mathbf{n}} \cong \mathbf{H}_{\mathbf{n}}^{d}$, con $n \geq 0$.

Por ser $\mathbf{P}$ un poset 3-irreducible tenemos que $\mathbf{P}-x$ es $C I$. Sea $M$ un modelo $C I$ de $\mathbf{P}-x$ sobre un camino huésped $P$. Sea $x^{\prime} \in X$ tal que $x^{\prime}<: x$. Consideremos $W_{x^{\prime}}$ el camino del modelo $M$ correspondiente a $x^{\prime}$. Llamemos $q$ al vértice de $P$ extremo de $W_{x^{\prime}}$. Sea $T$ el árbol que se obtiene agregando a $P$ un vértice pendiente $q^{\prime}$ adyacente a $q$ y llamemos $W_{x}$ al camino de $T$ que se obtiene agregando el vértice $q^{\prime}$ al camino $W_{x^{\prime}}$. Agregando al modelo $M$ el camino $W_{x}$ se obtiene un modelo $C P T$ de $\mathbf{P}$ sobre el árbol $T$.

En caso contrario, si $\mathbf{P}$ no es ninguno de los posets citados anteriormente, y no siendo ninguno de los posets $\mathbf{I}_{\mathbf{n}}, n \geq 0$, se tiene que $\mathbf{Q}$ debe ser alguno de los siguientes posets: $\mathbf{B}, \mathbf{D}, \mathbf{D}^{d}, \mathbf{C X}_{\mathbf{2}}^{d}, \mathbf{C X}_{\mathbf{3}}, \mathbf{C X}_{\mathbf{3}}^{d}, \mathbf{F} \mathbf{X}_{\mathbf{1}}, \mathbf{F} \mathbf{X}_{\mathbf{2}}, \mathbf{F} \mathbf{X}_{\mathbf{2}}^{d}, \mathbf{A}_{\mathbf{n}} \cong \mathbf{A}_{\mathbf{n}}^{d}, \mathbf{I}_{\mathbf{n}}^{d}, \mathbf{G}_{\mathbf{n}} \cong \mathbf{G}_{\mathbf{n}}^{d} \mathrm{o}$ $\mathbf{J}_{\mathbf{n}} \cong \mathbf{J}_{\mathbf{n}}^{d}$, con $n \geq 0$. En las Figuras 4.2 y 4.3 exponemos un modelos $C P T$ para cada uno de estos posets. Chequear la exactitud de estos modelos es una tarea que requiere tiempo pero es sencilla.

Veremos a continuación que los posets $\mathbf{T o p}_{\mathbf{3} \text {-irred }}$ permiten caracterizar a los posets $C P T$ dentro de cualquier clase de posets en la cual la condición necesaria para ser CPT sea también suficiente.

Teorema 4.1.5. Sea $\mathcal{A}$ una clase de posets donde la condición necesaria para ser CPT establecida por el Lema 4.1.2 es también suficiente.

Un poset $\mathbf{P} \in \mathcal{A}$ es $C P T$ si y solamente si $\mathbf{P}$ es $\mathbf{T o p}_{\mathbf{3} \text {-irred-libre. }}$

Demostración: Sea $\mathbf{P} \in \mathcal{A}$. Si $\mathbf{P}$ es $C P T$ entonces cumple con la condición necesaria para ser CPT. Luego, el conjunto descendente de cada vértice es $C I$. Resulta que $\mathbf{P}$ 

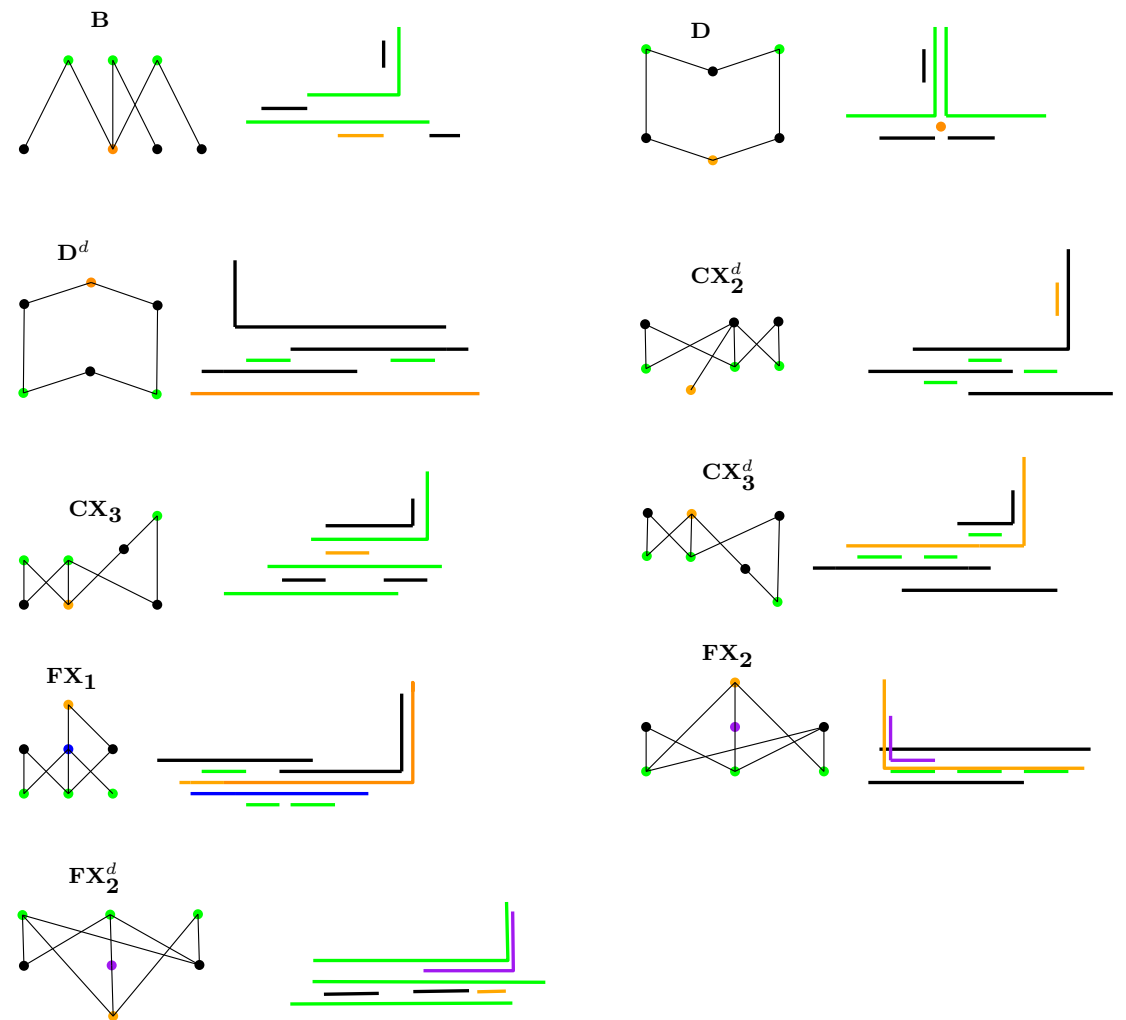

Figura 4.2: Representación de los modelos $C P T$ de los posets 3-irreducibles $\mathbf{B}, \mathbf{D}$, $\mathbf{D}^{d}, \mathbf{C X}_{2}^{d}, \mathbf{C X}_{3}, \mathbf{C X}_{3}^{d}, \mathbf{F X}_{1}, \mathbf{F X}_{2}$ y $\mathbf{F X}_{2}^{d}$. 

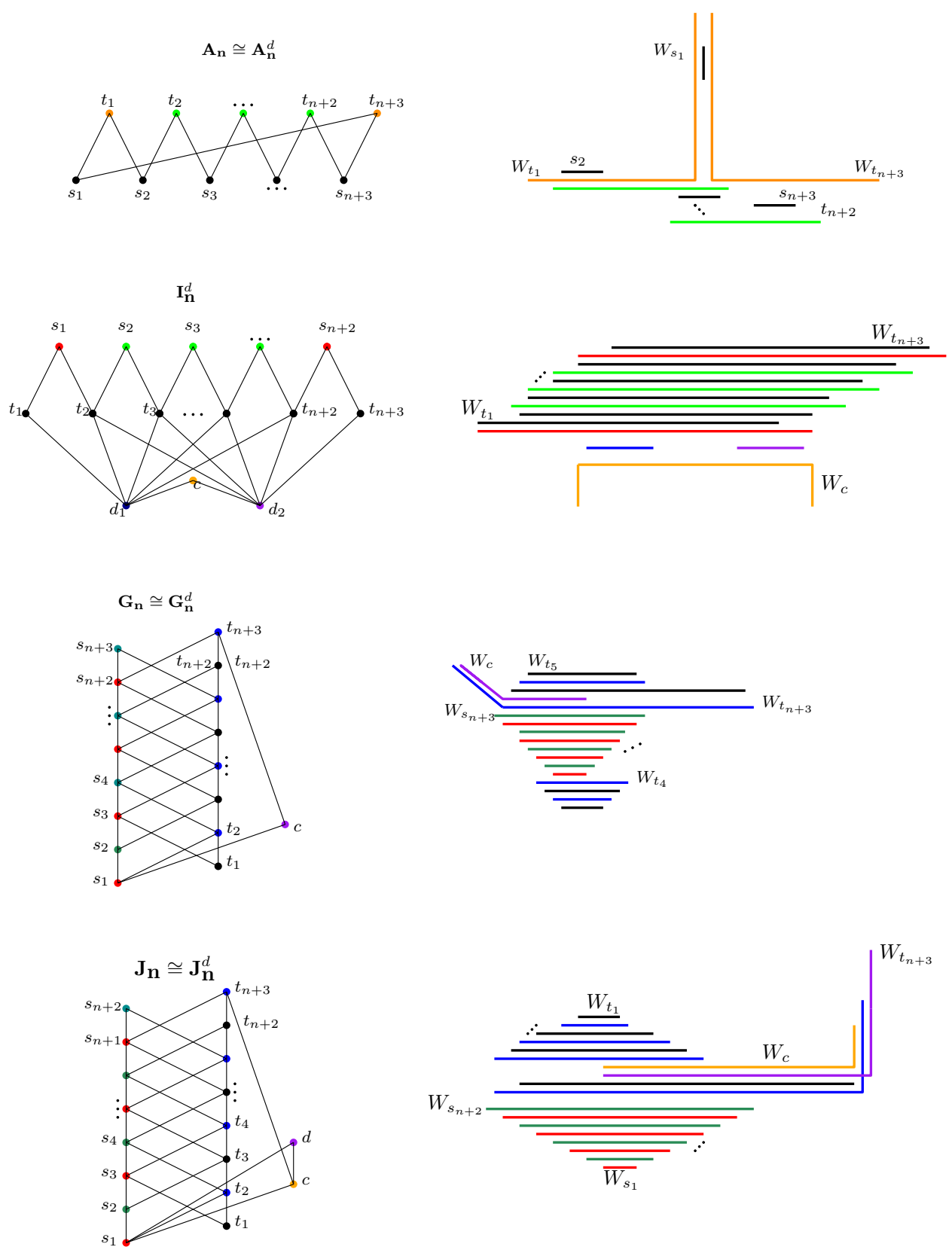

Figura 4.3: Representación de los modelos $C P T$ de los posets 3-irreducibles $\mathbf{A}_{\mathbf{n}} \cong \mathbf{A}_{\mathbf{n}}^{d}$, $\mathbf{I}_{\mathbf{n}}^{d}, \mathbf{G}_{\mathbf{n}} \cong \mathbf{G}_{\mathbf{n}}^{d}$ y $\mathbf{J}_{\mathbf{n}} \cong \mathbf{J}_{\mathbf{n}}^{d}$, con $n \geq 0$. 
es Top $_{3-\text { irred-libre. }}$

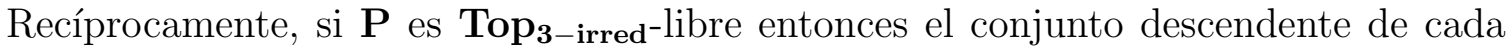
vértice debe ser $C I$, es decir $\mathbf{P}$ satisface la condición necesaria para ser $C P T$. Como en la clase $\mathcal{A}$ la condición necesaria es también suficiente, tenemos que $\mathbf{P}$ es $C P T$.

Si se desea obtener una caracterización mediante una familia de prohibidos minimales, basta reemplazar en el teorema anterior la familia $\mathbf{T o p}_{\mathbf{3} \text {-irred }}$ por la familia $\mathcal{C}_{\mathcal{A}}$ que se obtiene eliminando de $\mathbf{T o p}_{\mathbf{3} \text {-irred }}$ los posets $\widehat{\mathbf{I}_{\mathbf{n}}}$ para todo $n \geq 0$ y los posets que no pueden ser subposets de un miembro de la clase $\mathcal{A}$.

El Teorema 4.1.5 se usará para la caracterización por subposets prohibidos de dos clases especiales de posets en los Capítulos 5 y 6 .

A continuación mostraremos otros ejemplos particulares de posets $C P T$ y de posets que no son $C P T$. Utilizaremos las siguientes observaciones cuyas demostraciones son inmediatas.

Observación 4.1.6. Si en un modelo CPT dos caminos $W_{x}$ y $W_{y}$ son iguales, entonces los vértices $x$ e y son falsos gemelos. Luego, la propiedad de ser un poset CPT es invariante bajo la operación de adición de falsos gemelos.

Observación 4.1.7. Dados tres intervalos de una recta, existen dos de ellos tales que si I es un intervalo que contiene a ambos entonces I también contiene al restante tercer intervalo.

Observación 4.1.8. Sea $\left(W_{i}\right)_{1 \leq i \leq n}$ un modelo $C I$ de un poset $\mathbf{P}$ cuyo grafo de comparabilidad es un camino sin cuerdas $\left[v_{1}, v_{2}, \ldots, v_{n}\right]$, siendo $W_{i}$ el intervalo correspondiente a $v_{i}$. Si $v_{2}<v_{1}$ en $\mathbf{P}$ entonces :

1. de izquierda a derecha (o de derecha a izquierda) los intervalos correspondiente a los vértices maximales aparecen en orden $W_{1}, W_{3}, W_{5}, \ldots$ y los intervalos correspondiente a los vértices minimales aparecen en orden $W_{2}, W_{3}, W_{6}, \ldots$. 

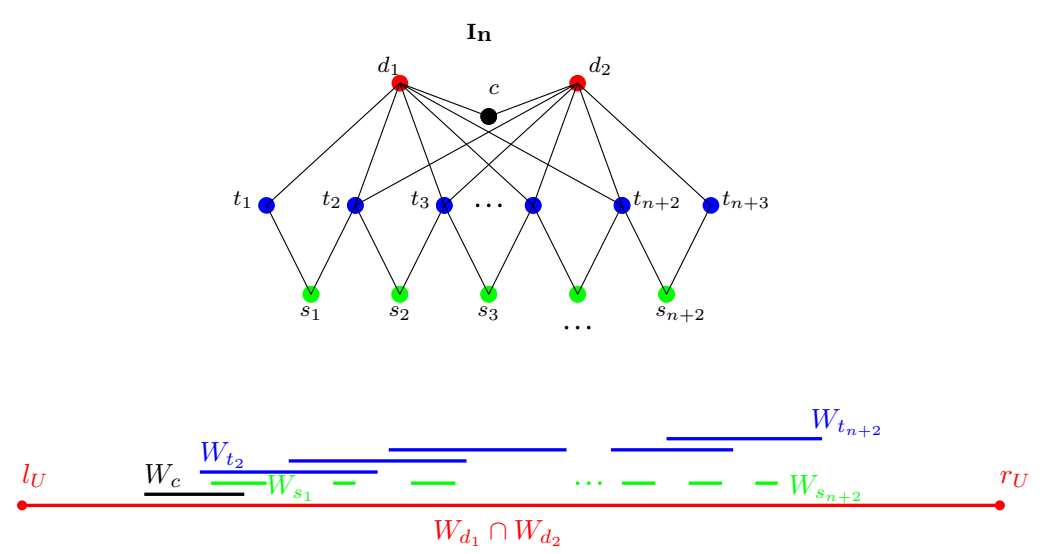

Figura 4.4: Poset 3-irreducible $\mathbf{I}_{\mathbf{n}}$ y un esquema de un modelo CPT que usaremos para probar que $\mathbf{I}_{\mathbf{n}}$ no es $C P T$ para $n \geq 0$, Lema 4.1.9.

2. No hay dos intervalos iguales, salvo que $n=3$ en cuyo caso puede ser $W_{1}=W_{3}$.

Lema 4.1.9. Sea $n \geq 0$. El poset 3-irreducible $\mathbf{I}_{\mathbf{n}}$ descripto en la Figura 4.4 no es CPT.

\section{Demostración:}

Supongamos que existe un modelo $C P T\left(W_{x}\right)_{x \in X}$ de $\mathbf{I}_{n}$, donde $X$ es el conjunto de vértices del poset nombrados como se indica en la Figura 4.4. Sea $A$ el conjunto de vértices $D\left(d_{1}\right) \cap D\left(d_{2}\right)$. Es claro que el subposet $\mathbf{I}_{\mathbf{n}}(A)$ es $C I$ y un modelo del mismo está contenido en el camino $U=W_{d_{1}} \cap W_{d_{2}}$. En particular los caminos $W_{t_{2}}$, $W_{t_{n+2}}$ y $W_{c}$ están contenidos en $U$. Representando a $U$ como un intervalo horizontal con extremos derecho e izquierdo $r_{U}$ y $l_{U}$, respectivamente; llamaremos $r_{v}$ y $l_{v}$ a los extremos derecho e izquierdo respectivamente del camino $W_{v}$ con $v \in A$. Sin pérdida de generalidad asumamos que $l_{t_{2}}$ está más cercano a $l_{U}$ que el extremo $l_{t_{n+2}}$, ver figura 4.4 .

Por la Observación 4.1.8 y el hecho que ningún camino $W_{v}$ con $v \in A-\{c\}$ puede contener o estar contenido en $W_{c}$, resulta que $l_{c}$ está entre $l_{U}$ y $l_{t_{2}}$ o $r_{c}$ está entre $r_{t_{n+2}}$ y $r_{U}$. Por simetría, podemos asumir, sin pérdida de generalidad, que $l_{c}$ está entre $l_{U}$ y $l_{t_{2}}$ y de esa forma $r_{c}$ debe estar entre $l_{c} \mathrm{y} r_{s_{1}}$. 

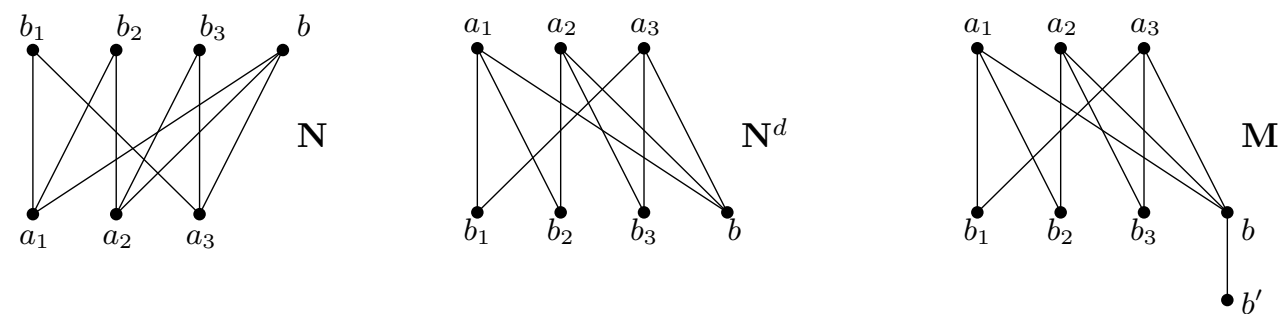

Figura 4.5: El poset $\mathbf{N}$ no es $C P T$. Su dual $\mathbf{N}^{d}$ admite un modelo $C P T$. El poset $\mathbf{M}$ obtenido a partir de $\mathbf{N}^{d}$ por la adición de $b^{\prime}$ no es $C P T$. Notar que $b$ y $b^{\prime}$ son gemelos.

Por otra parte, el camino $W_{t_{1}}$ debe estar contenido en $W_{d_{1}}$, debe contener a $W_{s_{1}}$ y no estar contenido en $U$, por lo que $l_{U}$ debe ser un punto interior del camino $W_{t_{1}}$ y de esa forma $W_{c}$ resulta estar contenido en $W_{t_{1}}$, contradiciendo el hecho que $c$ y $t_{1}$ son incomparables.

Lema 4.1.10. El poset $\boldsymbol{N}^{d}$ representado en la Figura 4.5 es CPT. En todo modelo $C P T$ de $\boldsymbol{N}^{d}$, el vértice b es representado por un camino formado por un vértice.

Demostración: En la Figura 4.6, hay un modelo $C P T$ de $\mathbf{N}^{d}$, lo que nos permite afirmar él es un poset $C P T$.

Sea $T$ el árbol huésped de algún modelo $C P T\left(W_{x}\right)_{x \in X}$ de $\mathbf{N}^{d}$ siendo $X=\left\{a_{1}, a_{2}, a_{3}, b, b_{1}, b_{2}, b_{3}\right\}$.

Por la Observación 4.1.6, dos vértices distintos no pueden ser asignados en un mismo camino de $T$. Sea $W_{a_{1}}:\left[q_{1}, q_{2}, \ldots, q_{k}\right]$ el camino que representa al vértice $a_{1}$, donde los $q_{i}$ son vértices de $T$. Ya que $b_{1}<a_{1}, b_{2}<a_{1}$ y $b<a_{1}$ en $\mathbf{N}^{d}$, se sigue que $W_{b_{1}}, W_{b_{2}}$ y $W_{b}$ son subcaminos de $W_{a_{1}}$. Para $1 \leq i \leq j \leq k$, denotamos $\left[q_{i}, q_{j}\right]$ al subcamino de $W_{a_{1}}$ con vértices $q_{i}, q_{i+1}, \ldots, q_{j-1}, q_{j}$.

De acuerdo a las relaciones que satisfacen los elementos de $\mathbf{N}^{d}$ se cumple que $W_{b} \subseteq$ $W_{a_{1}} \cap W_{a_{2}} \cap W_{a_{3}}$ y $W_{b_{1}} \subset W_{a_{1}} \cap W_{a_{3}}$ pero $W_{b_{1}} \not \subset W_{a_{2}}$. Además, $W_{b_{2}} \subset W_{a_{1}} \cap W_{a_{2}}$ pero $W_{b_{2}}$ no está contenido en $W_{a_{3}}$. Entonces, sin pérdida de generalidad, podemos asumir que $i_{b_{1}} \leq j_{b_{1}}<j_{b}<j_{b_{2}}$ y $i_{b_{1}}<i_{b}<i_{b_{2}} \leq j_{b_{2}}$. 


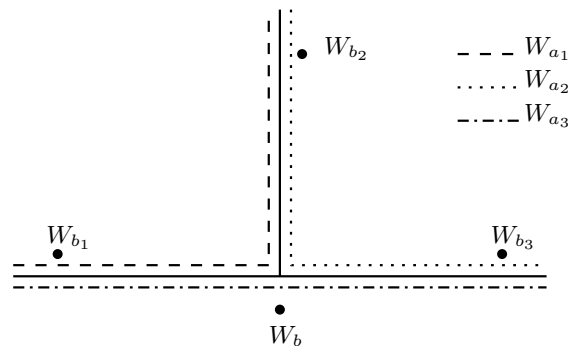

Figura 4.6: Un modelo $C P T$ de $\mathbf{N}^{d}$. Los vértices $b_{1}, b_{2}, b_{3}$ y $b$ son representados por caminos con un vértice.

Ya que $W_{b_{3}}$ no está contenido en $W_{a_{1}}$ existe un vértice $h$ de $T$ que pertenece a $\left(W_{a_{3}} \cap W_{a_{2}}\right)-W_{a_{1}}$. Sea $q_{i_{h}}$ el vértice de $W_{a_{1}}$ más cercano a $h$ en $T$. Ya que $h$ es un vértice de $W_{a_{3}}$, y $W_{b_{1}}$ y $W_{b}$ están contenidos en $W_{a_{3}}$, entonces $i_{h} \leq i_{b_{1}}$ o $i_{h} \geq j_{b}$. Si $i_{h} \leq i_{b_{1}}$, ya que $h$ es un vértice de $W_{a_{2}}$ y $W_{b_{2}}$ están contenidos en $W_{a_{2}}$, tenemos que $W_{b_{1}}$ está contenido en $W_{a_{2}}$, lo cual contradice que $b_{1}$ y $a_{2}$ son incomparables en $\mathbf{N}^{d}$. Por lo tanto, $j_{b} \leq i_{h}$. Análogamente, debido a que $h$ es un vértice de $W_{a_{2}}$, y $W_{b_{2}}$ y $W_{b}$ está contenido en $W_{a_{2}}$, entonces $i_{h} \leq i_{b}$ o $i_{h} \geq j_{b_{2}}$. Si $i_{h} \geq j_{b_{2}}$, ya que $h$ es un vértice de $W_{a_{3}}$ y $W_{b_{1}}$ está contenido en $W_{a_{3}}$, tenemos que $W_{b_{2}}$ está contenido en $W_{a_{3}}$, lo cual contradice que $a_{3}$ y $b_{2}$ son incomparables in $\mathbf{N}^{d}$. Por lo tanto, $i_{h} \leq i_{b}$. Concluimos que $i_{b}=j_{b}=i_{h}$, por lo que $W_{b}$ es un camino formado por un único vértice.

Lema 4.1.11. Sean N y $\mathbf{M}$ los posets representados en la Figura 4.5. Las siguientes afirmaciones son ciertas:

1. El poset $\mathbf{N}$ satisface la condición necesaria para ser CPT establecida en el Lema 4.1.2, pero $\mathbf{N}$ no es CPT.

2. El poset $\mathbf{N}^{d}$ es CPT, pero el poset $\mathbf{M}$ que se obtiene agregando a $\mathbf{N}^{d}$ el vértice $b^{\prime}$ gemelo de b no es CPT.

Demostración: Es claro que $\mathbf{N}$ satisface la condición necesaria. Asumamos, con el fin de llegar a una contradicción, que el poset $\mathbf{N}$ admite un modelo $C P T\left(W_{x}\right)_{x \in X}$, 
donde $X$ es el conjunto de vértices de $\mathbf{N}$. Como $a_{1}<b, a_{2}<b$ y $a_{3}<b$ en $\mathbf{N}$, tenemos que $W_{a_{1}}, W_{a_{2}}$ y $W_{a_{3}}$ son intervalos de $W_{b}$. Así, por la Observación 4.1.7, dos de ellos, asumamos sin pérdida de generalidad que son $W_{a_{1}}$ y $W_{a_{2}}$, satisfacen que todo subcamino de $W_{b}$ que contiene a $W_{a_{1}}$ y $W_{a_{2}}$ también contiene a $W_{a_{3}}$. Ya que $W_{b_{2}} \cap W_{b}$ es un subcamino de $W_{b}$ que contiene a $W_{a_{1}}$ y $W_{a_{2}}$, tenemos que $W_{a_{3}} \subseteq W_{b_{2}} \cap W_{b}$, por lo que $W_{a_{3}} \subseteq W_{b_{2}}$. Por otra parte, es claro que $W_{a_{3}} \neq W_{b_{2}}$, y así $W_{a_{3}} \subset W_{b_{2}}$, lo que contradice el hecho que $a_{3}$ y $b_{2}$ son incomparables en $\mathbf{N}$.

El segundo enunciado es una consecuencia directa del Lema 4.1 .10 y de que, en cualquier modelo $C P T$ de $\mathbf{M}$, el camino correspondiente a $b^{\prime}$ debe ser un subcamino propio del camino correspondiente a $b$.

Corolario 4.1.12. La condición necesaria para ser CPT dada por el Lemma 4.1.2 no es suficiente. La propiedad de ser un poset CPT se puede perder al agregar vértices gemelos. El hecho de que un poset sea CPT no implica que su dual también lo sea.

El siguiente lema será utilizado para la demostración de los Teoremas 6.1.8 y 6.2.8 en el Capítulo 6.

Lema 4.1.13. Sea $\boldsymbol{P}=(X, P)$ un poset $C P T$ y sin falsos gemelos. Si $\mathbf{P}$ admite un modelo CPT en el cual ningún camino se reduce a un vértice, entonces $\mathbf{P}$ admite un modelo CPT en el que ningún camino se reduce a un vértice y, además, no hay dos caminos del modelo con algún extremo coincidente.

Demostración: Sea $M=\left(W_{x}\right)_{x \in X}$ un modelo $C P T$ en un árbol huésped $T$, en el que todo camino del modelo tiene al menos dos vértices de $T$. Procederemos recursivamente disminuyendo la cantidad de caminos con extremos coincidentes. Si no hay tales caminos, la demostración es obvia. Observar que, como el poset no tiene vértices falsos gemelos, por la Observación 4.1.6 no hay dos caminos iguales. Luego dos caminos solo pueden compartir uno de sus extremos. 
Dado un vértice $q \in V(T)$, sea $W_{v}$ un camino de menor longitud entre los que comparten extremo $q$. Sean $q_{1}, q_{2}, \ldots, q_{k}$ los vecinos de $q$ en $T$. Como ningún camino se reduce a un único vértice, tenemos que $W_{v}$ debe contener algún otro vértice $q_{i}$, digamos $q_{1}$. Para obtener el nuevo modelo, procedemos subdividiendo la arista $q q_{1}$ de $T$ mediante el agregado de un vértice $q_{1}^{\prime}$ y haciendo la misma subdivisión en todos los caminos de $M$ que contienen a esa arista; reemplazando el camino $W_{v}$ por el camino $W_{v}^{\prime}$ que se obtiene removiéndole a $W_{v}$ el vértice extremo $q$ (en este caso el nuevo extremo de $W_{v}^{\prime}$ resulta ser $q_{1}^{\prime}$ y $W_{v}^{\prime}$ no se reduce a un vértice); los demás caminos de $M$ no se modifican.

Como el único camino del nuevo modelo que contiene al vértice $q_{1}^{\prime}$ es $W_{v}^{\prime}$, es claro que el número de caminos con extremos coincidentes ha disminuido. Veamos que la relación de contención entre los caminos no se ha modificado. Efectivamente, los únicos cambios que podrían ocurrir son: que $W_{v}^{\prime}$ quede propiamente contenido en un camino en el cual $W_{v}$ no lo estaba, lo cual claramente no es posible; o que $W_{v}^{\prime}$ deje de contener propiamente un camino $W_{x}$ que $W_{v}$ contenía. Esto tampoco puede ocurrir pues en tal caso $q$ debió ser también vértice extremo de $W_{x}$ y tener menor longitud que $W_{v}$, contradiciendo su elección.

\subsection{Subclases}

Debido a que todo poset y su dual son asociados, el Lema 4.1.12 muestra que la propiedad fuerte de los poset $C I$ enfatizada en la Observación 2.3.5 no es cierta para los posets $C P T$, es decir, el hecho que un poset $\mathbf{P}^{\prime}$ esté asociado con un poset $C P T$ $\mathbf{P}$ no implica que $\mathbf{P}^{\prime}$ sea $C P T$. Más aún, el hecho que $\mathbf{P}$ sea $C P T$ no implica que $\mathbf{P}^{d}$ sea $C P T$. Esto motiva las siguientes definiciones: 
Definición 4.2.1. Un poset $\mathbf{P}$ es dualmente-CPT si $\mathbf{P}$ y $\mathbf{P}^{d}$ son $C P T$.

Definición 4.2.2. Un poset $\mathbf{P}$ es fuertemente-CPT si $\mathbf{P}$ es CPT y todo otro poset asociado con $\mathbf{P}$ también es CPT.

Indicaremos dually- $C P T$ (fuertemente- $C P T$ ) a la clase de posets dually- $C P T$ (fuertemente$C P T)$. Es claro que

$$
C I \subseteq \text { fuertemente- } C P T \subseteq \text { dualmente- } C P T \subseteq C P T .
$$

Notar que la última inclusión en (1) es estricta: el poset $\mathbf{N}^{d}$ en el Lema 4.1.11 es CPT pero no es dualmente- $C P T$.

Consideremos el poset $\mathbf{B}$ cuyo diagrama de Hasse está representado en la Figura 4.1. $\mathbf{B}$ es $C P T$, pero no es $C I$ (observar que $\mathbf{B}$ es uno de los posets 3-irreducibles representados en la figura 2.3). El grafo de comparabilidad de $\mathbf{B}$ es un árbol, luego la clase de posets cuyos grafos de comparabilidad son un árbol no está contenida en la clase de posets $C I$. El siguiente teorema muestra que tales posets son fuertementeCPT y, consecuentemente, la primera inclusión en (1) es estricta.

Teorema 4.2.3. Todo poset cuyo grafo de comparabilidad es un árbol es fuertementeCPT.

Demostración: Sea $\mathbf{P}$ un poset tal que su grafo de comparabilidad $G_{\mathbf{P}}$ es un árbol. Notar que $\mathbf{P}$ tiene altura dos, por lo cual todo vértice de $\mathbf{P}$ es un elemento minimal o un elemento maximal. Se construirá inductivamente un modelo $C P T$ inyectivo de $\mathbf{P}$ en el cual cada vértice minimal está representado por un camino con un único vértice. Sean $v_{0}$ una hoja de $G_{\mathbf{P}}$ y $\left(W_{v}\right)_{v \in V-\left\{v_{0}\right\}}$ un modelo inyectivo $C P T$ de $G_{\mathbf{P}}-v_{0}$ sobre un árbol huésped $T$. Llamemos $v_{1}$ al único vértice de $\mathbf{P}$ comparable con $v_{0}$. Tenemos dos casos a considerar:

Si $v_{0}$ es minimal en $\mathbf{P}$ entonces $v_{1}$ es maximal, luego $W_{v_{1}}$ no está contenido en ningún otro camino del modelo. Sea $q \in V(T)$ un vértice extremo de $W_{v_{1}}$. Procedemos 
adicionando a $T$ un nuevo vértice $q^{\prime}$ adyacente a $q$, reemplazando $W_{v_{1}}$ por $W_{v_{1}} \cup\left\{q^{\prime}\right\}$, haciendo $W_{v_{0}}=\left\{q^{\prime}\right\}$ y tomando los restantes caminos sin cambios.

Si $v_{0}$ es maximal en $\mathbf{P}$ entonces $v_{1}$ es minimal, por lo que $W_{v_{1}}$ es un camino con un único vértice en $T$, digamos $W_{v_{1}}=\{q\}$, donde $q \in V(T)$. En este caso, procedemos agregando a $T$ un nuevo vértice $q^{\prime}$ adyacente a $q$, haciendo $W_{v_{0}}=\left\{q, q^{\prime}\right\}$ y tomando los restantes caminos sin cambios.

Determinar si la inclusión central en (1) es estricta o no permanece como un problema abierto.

Problema abierto 4.2.4. ¿Existe un poset dualmente-CPT que no sea fuertemente-CPT?

Notar que la propiedad de ser fuertemente-CPT no es necesariamente hereditaria por subposets, podría suceder que un subposet $\mathbf{P}^{\prime}$ de $\mathbf{P}$ admita más posets asociados que el mismo $\mathbf{P}$. Sin embargo no sabemos si este es el caso para los posets que son fuertemente- $C P T$.

Problema abierto 4.2.5. ¿La propiedad de ser fuertemente-CPT es hereditaria por subposets?

Claramente, una respuesta negativa para el Problema 4.2.4 implica una respuesta positiva al Problema 4.2.5. En los dos capítulos siguientes, resolvemos ambos problemas para la clase de posets split y la clase de posets $k$-tree.

\subsection{Dimensión de los posets $C P T$}

Los posets que admiten un modelo por contención de intervalos en una recta, llamados posets $C I$, son exactamente los posets con dimensión a lo sumo dos. Una pregunta natural que plantea J. Spinrad en [19] es si la dimensión de los posets que admiten 


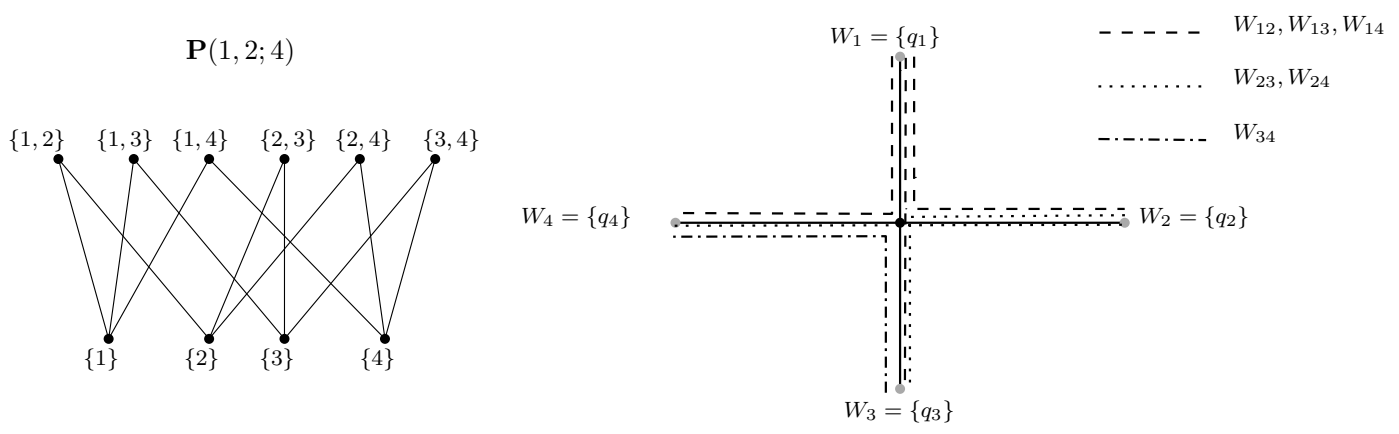

Figura 4.7: Diagrama de Hasse del poset $\mathbf{P}(1,2 ; 4)$ y un modelo $C P T$ del mismo.

un modelo por contención de caminos en un árbol, o sea la dimensión de los posets CPT, también está acotada superiormente por alguna constante. En esta sección, mediante el Teorema 4.3.1, responderemos negativamente a esta pregunta. También consideraremos el problema de determinar la existencia de una cota superior para la dimensión de intervalos de los posets $C P T$.

Siguiendo la notación de [23], para $k$ y $n$ enteros positivos, $\mathbf{P}(1, k ; n)$ indica el poset cuyos elementos son los subconjuntos con exactamente un elemento y los subconjuntos con exactamente $k$ elementos de $\{1,2,3, \ldots, n\}$, ordenados por inclusión.

Los posets $\mathbf{P}(1, n-1 ; n)$ (también conocidos como posets de Hiraguchi) son ejemplos estándar de posets con dimensión tan grande como se quiera eligiendo $n$ adecuadamente. De hecho $\operatorname{dim}(\mathbf{P}(1, n-1 ; n))=n[23]$. Es de destacar que estos posets no pueden ser utilizados para responder la pregunta de Spinrad, pues ellos no son posets $C P T$. Efectivamente, si $n \geq 4$, el poset $\mathbf{N}$ representado en la Figura 4.5 es un subposet de $\mathbf{P}(1, n-1 ; n)$, luego, por el Lema 4.1.11, $\mathbf{P}(1, n-1 ; n)$ no es $C P T$.

En la prueba del Teorema 4.3 .1 usamos que los posets $\mathbf{P}(1,2 ; n)$ son $C P T$. Como ejemplo, en la Figura 4.7 representamos al poset $\mathbf{P}(1,2 ; 4)$ junto con un modelo $C P T$ del mismo donde el árbol huésped es una estrella con cuatro hojas.

Teorema 4.3.1. Para todo entero positivo d, existe un poset $C P T \mathbf{P} \operatorname{con} \operatorname{dim}(\mathbf{P})>d$.

Demostración: Primero demostraremos que, para cada $n \geq 1$, el poset $\mathbf{P}(1,2 ; n)$ 
es $C P T$. Sea $T$ una estrella con centro $q$ y hojas $q_{1}, q_{2}, \ldots, q_{n}$. Cada subconjunto formado por un elemento $\{i\}$ está representado por el camino $W_{i}=\left\{q_{i}\right\}$ de $T$, y cada subconjunto de 2 elementos $\{i, j\}$ está representado por el camino $W_{i j}=\left\{q_{i}, q, q_{j}\right\}$. Usando el hecho de que $\operatorname{dim}(\mathbf{P}(1,2 ; n))>\log _{2} \log _{2} n$ para $n \geq 3$ [23], queda probado el teorema.

Un poset $\mathbf{Q}=(X, Q)$ se dice un orden de intervalos si existe una función $F$ asignando a cada elemento $x \in X$ un intervalo cerrado no degenerado $F(x)=\left[a_{x}, b_{x}\right]$, con $a_{x}<b_{x}$, de la recta real $\mathbb{R}$ tal que $x<y$ en $\mathbf{Q}$ si y solo si $b_{x}<a_{y}$ en $\mathbb{R}$. Todo poset es subposet de algún orden de intervalos. La dimensión de intervalos de un poset $\mathbf{P}$, denotada por $\operatorname{Idim}(\mathbf{P})$, es el menor entero positivo $k$ para el cual existen $k$ extensiones $Q_{1}, Q_{2}, \ldots, Q_{k}$ de $\mathbf{P}$ tales que se satisfacen las siguientes condiciones: 1.) $P=\bigcap_{i=1}^{k} Q_{i}$ y 2.) $\left(X, Q_{i}\right)$ es un orden de intervalos para $i=1,2, \ldots, k$.

Ya que los ordenes lineales son ordenes de intervalos, se tiene que $\operatorname{Idim}(\mathbf{P}) \leq \operatorname{dim}(\mathbf{P})$ para cada poset $\mathbf{P}$.

La diferencia entre ambas dimensiones puede ser grande; sin embargo, si $\mathbf{P}$ es un poset cuya altura es dos entonces esa diferencia es a lo sumo uno [24]. Luego, dado que los posets $\mathbf{P}(1,2 ; n)$ usados en la demostración del Teorema 4.3.1 tienen altura dos, obtenemos el siguiente teorema.

Teorema 4.3.2. Para todo entero positivo $d$, existe un poset CPT $\mathbf{P}$ satisfaciendo que $\operatorname{Idim}(\mathbf{P})>d$.

W. Trotter y J. Moore probaron el siguiente resultado que usaremos en la demostración del Teorema 4.3.4. Recordemos que un vértice $x$ de un grafo conexo $G$ es vértice de corte si $G-x$ es inconexo.

Teorema 4.3.3 ([23]). Sea $G=(V, E)$ un grafo conexo con dos o más vértices. Sea $\mathbf{S}=(X, S)$ el poset donde $X$ es el conjunto de todos los subgrafos inducidos de $G$ y 
$H_{1} \leq H_{2}$ en $P$ si y solo si $H_{1}$ es subgrafo inducido de $H_{2}$. Entonces la dimensión de $\mathbf{S}$ es igual a la cantidad de vértices de $G$ que no son vértices de corte de $G$.

Teorema 4.3.4. Si un poset $\mathbf{P}$ admite un modelo $C P T$ en un árbol huésped $T$ con $k$ hojas entonces $\operatorname{dim}(\mathbf{P}) \leq k$.

Demostración: Sea Q el poset formado por los subgrafos conexos del árbol $T$ ordenados por inclusión de vértices, es decir por la condición de ser subgrafo inducido. Por el Teorema 4.3.3 y el hecho que las $k$ hojas de $T$ son exactamente los vértices que no son de corte, tenemos que $\operatorname{dim}(\mathbf{Q})=k$. Como $\mathbf{P}$ es subposet de $\mathbf{Q}$, resulta que $\operatorname{dim}(\mathbf{P}) \leq \operatorname{dim}(\mathbf{Q})=k$.

El siguiente lema muestra que los posets usados en el Teorema 4.3.1 no son dualmente$C P T$, por lo tanto el siguiente problema permanece irresuelto:

Problema abierto 4.3.5. Determinar si la dimensión de los posets dualmente-CPT está acotada superiormente por una constante. La misma pregunta para los posets fuertemente-CPT.

Lema 4.3.6. Para todo $n \geq 4$, el poset dual de $\mathbf{P}(1,2 ; n)$, representado en la Figura 4.8, no es CPT.

Demostración: Observar que $\mathbf{P}(1,2 ; 4)$ es un subposet de $\mathbf{P}(1,2 ; n)$ para $n \geq 4$. Por lo tanto, es suficiente probar que $\mathbf{H}=\mathbf{P}(1,2 ; 4)^{d}$ no es $C P T$. Supongamos lo contrario y tomemos un modelo $C P T$ de $\mathbf{H}$. Para cada vértice $\{i\}$ o $\{i, j\}$ de $\mathbf{H}$, sean $W_{i}$, respectivamente $W_{i j}$, los correspondientes caminos en un árbol $T$. El camino $W_{1}$ contiene a los caminos $W_{12}, W_{13}$ y $W_{14}$, ver Figura 4.9. Notar que de a pares estos caminos tienen diferentes puntos extremos izquierdos y diferentes puntos extremos derechos. Por la simetría de $\mathbf{H}$, podemos asumir sin pérdida de generalidad que el punto extremo izquierdo de $W_{12}$ es el más cercano al punto extremo izquierdo de $W_{1}$ 


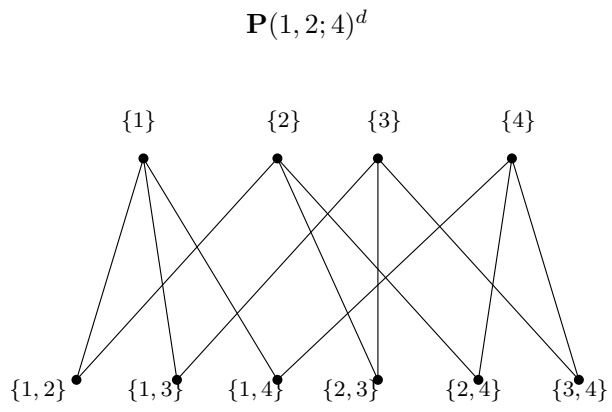

Figura 4.8: Poset $\mathbf{P}(1,2 ; 4)^{d}$.

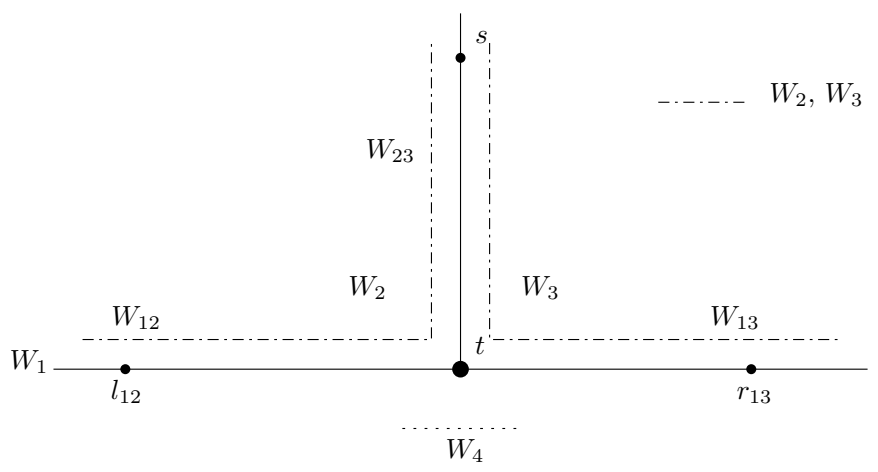

Figura 4.9: El poset $\mathbf{P}(1,2 ; 4)^{d}$ no es $C P T$.

y que el punto extremo derecho de $W_{13}$ es el más cercano al punto extremo derecho de $W_{1}$.

Notar que $W_{12} \subset W_{2}, W_{12} \nsubseteq W_{3}, W_{13} \subseteq W_{3}, W_{13} \nsubseteq W_{2}$, y por el vértice $\{2,3\}$, $W_{2} \cap W_{3} \nsubseteq W_{1}$. Por lo tanto, existe un vértice $t$ en el árbol huésped $T$ entre el vértice extremo derecho $r_{12}$ de $W_{12}$ y el vértice extremo izquierdo $l_{13}$ de $W_{13}$; y existe un vértice $s$ de $T$ adyacente a $t$ tal que el camino $W_{2}$ contiene a $r_{12}, t$ y $s$; mientras el camino $W_{3}$ contiene a $l_{13}, t$ y $s$. Ver Figura 4.9 .

Ya que $W_{14} \subset W_{1}, W_{14} \nsubseteq W_{2}$ y $W_{14} \nsubseteq W_{3}$, tenemos que el vértice extremo izquierdo de $W_{14}$ está entre $l_{12}$ y $t$; mientras el vértice extremo derecho de $W_{14}$ está entre $t$ y $r_{13}$; lo cual contradice la existencia del camino $W_{4}$. 


\section{Capítulo 5}

\section{Posets y grafos $C P T$ en la clase Split}

En este Capítulo demostramos que, en la clase de los posets split, la condición necesaria para ser un poset CPT dada en el Lema 4.1.2 es también suficiente. Como consecuencia obtenemos una caracterización por subposets prohibidos de los posets split CPT. Mostramos que un poset es split fuertemente-CPT si y solo si es dualmente- $C P T$; y los caracterizamos por subposets prohibidos. Obtenemos paralelamente una caracterización por subgrafos inducidos prohibidos de la clase de grafos split fuertemente- $C P T$, la cual coincide con la clase de grafos split dualmente- $C P T$.

\subsection{Posets Split}

Un grafo cuyo conjunto de vértices puede ser particionado en un clique y un conjunto estable es llamado split. La clase de grafos split es exactamente la clase de grafos $\left\{2 K_{2}, C_{4}, C_{5}\right\}$-libre [5]; por lo tanto un grafo es split y de comparabilidad si y solamente si ninguno de sus subgrafos inducidos es isomorfo a un grafo en la Figura 5.1. 

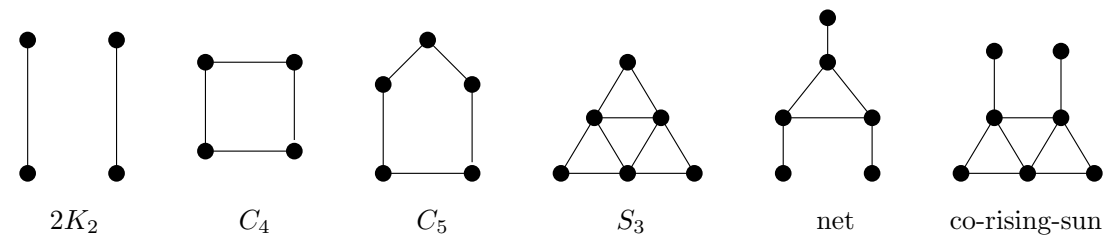

Figura 5.1: Subgrafos inducidos prohibidos para los grafos de comparabilidad split.

Definición 5.1.1. Un poset cuyo grafo de comparabilidad es split es llamado poset split.

Los posets split han sido estudiados, por ejemplo, en [14]. En la Figura 5.2 se ofrecen varios ejemplos de posets split.

Observación 5.1.2. Un poset $\mathbf{P}$ es split si y solo si su diagrama de Hasse consiste en una cadena maximal $x_{1}<x_{2}<\ldots x_{k}<y_{1}<y_{2}<\ldots<y_{m}$, llamada cadena principal de $\mathbf{P}$, y cualquier otro vértice $z$ de $\mathbf{P}$ satisface una de las siguientes condiciones:

- z tiene un único vecino, este vecino es alguno de los vértices yj en la cadena principal de $\mathbf{P}, y z<: y_{j}$. Observar que en este caso $z$ es minimal y está cubierto únicamente por $y_{j}$.

- $z$ tiene un único vecino, este vecino es alguno de los vértices $x_{i}$ en la cadena principal de $\mathbf{P}$, y $x_{i}<$ : z. Observar que en este caso $z$ es maximal y cubre únicamente a $x_{i}$.

- $z$ tiene exactamente dos vecinos, ellos son $x_{i}$ e $y_{j}$ en la cadena principal de $\mathbf{P}$, $y x_{i}<: z<: y_{j}$.

El vértice $y_{m}$ de la cadena principal es llamado vértice cima del poset.

En lo que sigue, los posets split que son $C P T$, dualmente- $C P T$ o fuertemente- $C P T$ serán caracterizados por subposets prohibidos. 

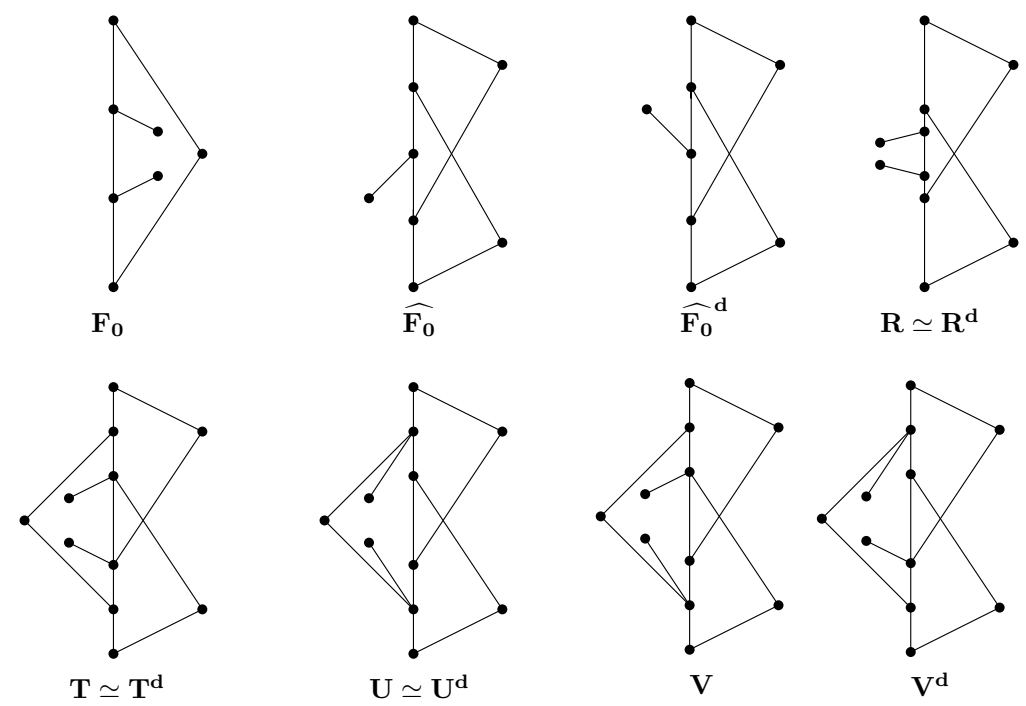

Figura 5.2: Ejemplos de posets split. $\mathbf{F}_{\mathbf{0}}$ no es $C I$ [23], pero $\mathbf{F}_{\mathbf{0}}$ es $C P T . \widehat{\mathbf{F}_{\mathbf{0}}}$ no es $C P T$, pero $\widehat{\mathbf{F}_{\mathbf{0}}} \mathbf{d}$ es $C P T$. Los posets $\mathbf{R}, \mathbf{T}, \mathbf{U}$ y $\mathbf{V}$ tienen a $\widehat{\mathbf{F}_{\mathbf{0}}}$ como subposet, luego no son $C P T$. Observar que los posets $\mathbf{R}, \mathbf{T}, \mathbf{U}$ y $\mathbf{V}$ también tienen a $\widehat{\mathbf{F}}_{\mathbf{0}}^{\mathbf{d}}$ como subposet.

Teorema 5.1.3. Sea $\mathbf{P}$ un poset split. $\mathbf{P}$ es $C P T$ si y solo si $\mathbf{P}(D[y])$ es $C I$, donde y es el vértice cima de $\mathbf{P}$.

Demostración: Por el Lema 4.1.2, si $\mathbf{P}$ es $C P T$ entonces $\mathbf{P}(D[x])$ es $C I$ para todo elemento $x$ de $\mathbf{P}$, en particular esto vale para el elemento $y$.

Recíprocamente sea $\mathbf{P}$ un poset split tal que $\mathbf{P}(D[y])$ es $C I$, siendo $y$ el vértice cima. Denotamos por $Z$ el conjunto de los vértices de $\mathbf{P}$ que no están en $D[y]$. Por la Observación 5.1.2, si $z \in Z$ entonces $z$ es maximal y existe un único vértice $x_{z}$ en la cadena principal adyacente a $z$; más aun, $x_{z}<: z$ en $\mathbf{P}$. Observar que distintos elementos $z$ y $z^{\prime}$ de $Z$ pueden ser adyacentes a un mismo vértice de la cadena principal, es decir, puede ocurrir $x_{z}=x_{z^{\prime}}$.

Sea $\left(W_{v}\right)_{v \in D[y]}$ un modelo $C I$ de $\mathbf{P}(D[y])$. Llamemos $P$ al camino huésped de este modelo. Por cada vértice $z \in Z$ agregamos a $P$ un vértice pendiente $q_{z}$ adyacente a un extremo del camino $W_{x_{z}}$. Llamemos $T$ al árbol resultante de este proceso. 
Obtenemos un modelo $C P T$ de $\mathbf{P}$ con árbol huésped $T$ agregando a la familia de caminos $\left(W_{v}\right)_{v \in D[y]}$ los caminos $W_{x_{z}} \cup\left\{q_{z}\right\}$ con $z \in Z$.

El Teorema 5.1.3 muestra que en la clase de los posets split la condición necesaria para ser CPT dada en el Lema 4.1.2 es también suficiente. Luego, el Teorema 4.1.5 y el siguiente lema completan la demostración del Teorema 5.1.5 que brinda una caracterización por subposets prohibidos para la clase de posets split $C P T$.

Lema 5.1.4. El único poset 3-irreducible que puede ser subposet de un poset split es el poset $\mathbf{F}_{\mathbf{0}}$ descripto en la Figura 5.2.

Demostración: Los posets 3-irreducibles son los posets descriptos en las Figuras 2.5 y 2.6 y sus respectivos duales. Sea $\mathbf{H}$ un poset 3-irreducible, subposet de un poset split.

Como los grafos split son cordales, es decir, no tienen ciclos sin cuerdas inducidos, entonces los posets split son acíclicos. Resulta que $\mathbf{H}$ no es ninguno de los posets $\mathbf{D}$, $\mathbf{C X}_{1}, \mathbf{C X}_{2}, \mathbf{C X}_{3}, \mathbf{E X}_{1}, \mathbf{E X}_{2}, \mathbf{F X} \mathbf{F}_{\mathbf{1}}, \mathbf{F} \mathbf{X}_{2}, \mathbf{A}_{\mathbf{n}}, \mathbf{I}_{\mathbf{n}}, \mathbf{G}_{\mathbf{n}}, \mathbf{J}_{\mathbf{n}}, \mathbf{H}_{\mathbf{n}}$ con $\mathbf{n} \geq 0$ ni sus respectivos duales.

Luego $\mathbf{H}$ debe ser alguno de los posets $\mathbf{B}, \mathbf{C}, \mathbf{E}_{\mathbf{n}} \circ \mathbf{F}_{\mathbf{n}}$, con $n \geq 0$. Es fácil verificar que el grafo de comparabilidad de cada uno de estos posets, salvo en el caso de $\mathbf{F}_{\mathbf{0}}$, tiene un $K_{2}$ como subgrafo inducido, y por ende no es split (recordar que los grafos split son los grafos $\left\{2 K_{2}, C_{4}, C_{5}\right\}$-libre).

Observar que el dual del poset $\mathbf{F}_{\mathbf{0}}$ es el mismo poset $\mathbf{F}_{\mathbf{0}}$. El poset $\widehat{\mathbf{F}_{\mathbf{0}}}$ que se obtiene agregando a $\mathbf{F}_{\mathbf{0}}$ un elemento máximo (ver Definición 4.1.3) se describe en la Figura 5.2 .

Teorema 5.1.5. Sea $\mathbf{P}$ un poset split. $\mathbf{P}$ es CPT si y solo si $\mathbf{P}$ no contiene el poset $\widehat{\mathbf{F}_{\mathbf{0}}}$ (representado en la Figura 5.2 como subposet). 
El siguiente corolario es una consecuencia inmediata del teorema previo.

Corolario 5.1.6. Los posets split $\mathbf{R}, \mathbf{T}, \mathbf{U}$ y $\mathbf{V}$ representados en la Figura 5.2 no son posets $C P T$.

Demostración: Cada uno de ellos tiene como subposet a $\widehat{\mathbf{F}_{\mathbf{0}}}$.

El segundo ítem del Corolario 2.3.9 no es aplicable al poset $\widehat{\mathbf{F}_{\mathbf{0}}}$ por cuanto este poset no es primo ( $\mathbf{F}_{\mathbf{0}}$ es un módulo no trivial), aunque la propiedad descripta por dicho corolario puede establecerse para $\widehat{\mathbf{F}_{\mathbf{0}}}$ de la siguiente manera.

Lema 5.1.7. Sean $\mathbf{P}$ y $\mathbf{P}^{\prime}$ posets asociados. Si $\widehat{\mathbf{F}_{\mathbf{0}}}$ (Figura 5.2) es un subposet de $\mathbf{P}$ entonces $\widehat{\mathbf{F}_{\mathbf{0}}}$ o $\widehat{\mathbf{F}}_{\mathbf{0}}^{d}$ es subposet de $\mathbf{P}^{\prime}$.

Demostración: Los módulos no triviales de $\widehat{\mathbf{F}_{\mathbf{0}}}$ son: su vértice cima, que llamaremos $y$, y los vértices del subposet $\mathbf{F}_{\mathbf{0}}$. Al conjunto de vértices de $\mathbf{F}_{\mathbf{0}}$ lo denotaremos por $X$.

Como $\mathbf{P}$ y $\mathbf{P}^{\prime}$ son asociados (tienen el mismo grafo de comparalibidad) podemos determinar el vértice $y^{\prime}$ y el conjunto $X^{\prime}$ de vértices de $\mathbf{P}^{\prime}$ correspondientes al vértice $y$ y al conjunto $X$, respectivamente.

Ya que $\mathbf{F}_{\mathbf{0}}$ es primo por el segundo ítem del Corolario 2.3.9 y porque $\mathbf{F}_{\mathbf{0}}=\mathbf{F}_{\mathbf{0}}{ }^{d}$, tenemos que el subposet de $\mathbf{P}^{\prime}$ inducido por los vértices de $X^{\prime}$ es $\mathbf{F}_{\mathbf{0}}$.

Observar que, en $\mathbf{P}^{\prime}$, el vértice $y^{\prime}$ debe ser mayor que todos los vértices de $X^{\prime}$ o debe ser menor que todos ellos, luego en $\mathbf{P}^{\prime}$ tenemos el subposet $\widehat{\mathbf{F}_{\mathbf{0}}}$ o el subposet $\widehat{\mathbf{F}}_{\mathbf{0}}^{d}$ (pues $\mathbf{F}_{\mathbf{0}}=\mathbf{F}_{\mathbf{0}}{ }^{d}$ ).

Teorema 5.1.8. Sea $\mathbf{P}$ un poset split. Las siguientes condiciones son equivalentes.

i) $\mathbf{P}$ es fuertemente-CPT. 
ii) $\mathbf{P}$ es dualmente-CPT.

iii) $\mathbf{P}$ no contiene a $\widehat{\mathbf{F}_{\mathbf{0}}}$ ni a $\widehat{\mathbf{F}}_{\mathbf{0}}^{d}$ como subposets.

Demostración: La implicación $i$ ) entonces $i i$ ) es cierta incluso para posets que no son split, y ii) implica $i i i)$ es consecuencia del Teorema 5.1.5.

Sea $\mathbf{P}$ un poset split que satisface $i i i)$. Por el Teorama 5.1.5, $\mathbf{P}$ es CPT. Asumamos, con el objetivo de llegar a una contradicción, que $\mathbf{P}$ no es fuertemente-CPT. Sea $\mathbf{P}^{\prime}$ un poset no $C P T$ asociado a $\mathbf{P}$. Por el Teorema 5.1.5, $\mathbf{P}^{\prime}$ contiene al poset $\widehat{\mathbf{F}_{\mathbf{0}}}$ como subposet; luego, por el Lema 5.1.7, $\mathbf{P}$ contiene a $\widehat{\mathbf{F}_{\mathbf{0}}}$ o $\widehat{\mathbf{F}}_{\mathbf{0}}^{d}$ como subposet, lo que contradice nuestra asunción.

Observar que, por el Lema 5.1.7, si un poset $\mathbf{P}$ tiene a $\widehat{\mathbf{F}_{\mathbf{0}}}$ como subposet, entonces todo poset asociado a $\mathbf{P}$ tiene a $\widehat{\mathbf{F}_{\mathbf{0}}}$ o a $\widehat{\mathbf{F}}_{\mathbf{0}}^{d}$ como subposet. Nos interesaría mejorar ese resultado caracterizando los posets tales que si ellos tienen a $\widehat{\mathbf{F}_{\mathbf{0}}}$ como subposet entonces cada uno de sus asociados tienen a $\widehat{\mathbf{F}_{\mathbf{0}}}$ como subposet. El siguiente resultado, que será utilizado en la próxima sección en la caracterización de los grafos split que son $C P T$, muesta ejemplos de tales posets.

Lema 5.1.9. Sea $\mathbf{P}$ alguno de los posets $\mathbf{R}, \mathbf{T}, \mathbf{U}$ o $\mathbf{V}$ descriptos en la Figura 5.2. Si $\mathbf{P}^{\prime}$ es un poset asociado a $\mathbf{P}$ entonces $\mathbf{P}^{\prime}$ tiene a $\widehat{\mathbf{F}_{\mathbf{0}}}$ como subposet.

Demostración: Podemos ver que el grafo de comparabiliad de $\mathbf{P}$ (se describe en la Figura 5.1 para los distintos $\mathbf{P}$ ) tiene una única clase de implicancia. Luego, por la Proposición 2.3.2, $\mathbf{P}$ tiene a su dual como único poset asociado. Por lo tanto, podemos asumir $\mathbf{P}^{\prime}=\mathbf{P}^{d}$.

Por otra parte, es fácil ver en la Figura 5.2 que $\mathbf{P}$ tiene a $\widehat{\mathbf{F}_{\mathbf{0}}}$ y a $\widehat{\mathbf{F}}_{\mathbf{0}}^{\mathbf{d}}$ como subposets. Luego, $\mathbf{P}^{d}$ tiene a $\widehat{\mathbf{F}_{\mathbf{0}}}$ como subposet y la demostración está completa. 

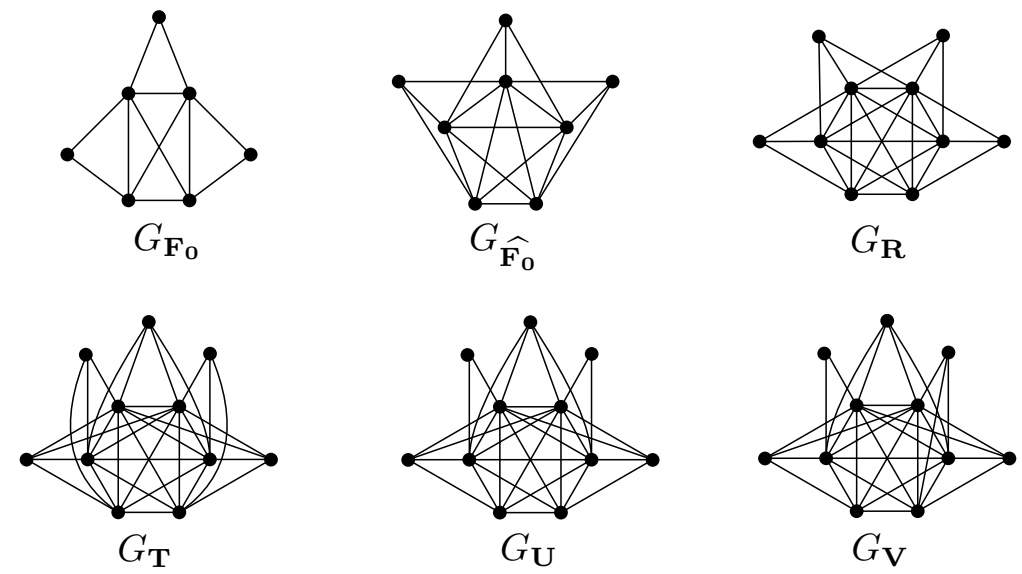

Figura 5.3: Los grafos de comparabilidad de los posets $\mathbf{F}_{\mathbf{0}}, \widehat{\mathbf{F}_{\mathbf{0}}}, \mathbf{R}$ y $\mathbf{T}, \mathbf{U}, \mathbf{V}$ en la Figura 5.2.

\subsection{Grafos $C P T$ Split}

Los grafos de comparabilidad de los posets $C P T$ son llamados grafos CPT o grafos de contención de caminos en un árbol.

Claramente, un grafo $G=(V, E)$ es $C P T$ si y solo si existe una orientación transitiva $\vec{E}$ de $G$, un árbol $T$ y una familia $\left(W_{v}\right)_{v \in V}$ de caminos de $T$ satisfaciendo que

$$
\overrightarrow{u v} \in \vec{E} \Leftrightarrow W_{u} \subset W_{v}
$$

Los grafos fuertemente- $C P T$ y dualmente- $C P T$ son los grafos de comparabilidad de los posets fuertemente- $C P T$ y dualmente- $C P T$, respectivamente.

No todo grafo split es de comparabilidad. Los grafos split de comparabilidad están caracterizados por subgrafos prohibidos (ver Figura 5.1). Por otra parte, no todo grafo split de comparabilidad admite un modelo $C I$. Por comparación de la familia de subgrafos inducidos prohibidos para grafos split y para grafos $C I$, puede establecerse que un grafo split es $C I$ si y solo si no contiene a $G_{\mathbf{F}_{\mathbf{0}}}$ en la Figura 5.3 como subgrafo inducido.

En el siguiente Teorema presentamos una caracterización de los grafos split que son 
dualmente- $C P T$ y probamos que son exactamente los grafos split que son fuertementeCPT.

Teorema 5.2.1. Sea $G$ un grafo de comparabilidad split. Las siguientes afirmaciones son equivalentes:

i) G es fuertemente-CPT.

ii) G es dualmente-CPT.

iii) $G$ no contiene a $G_{\widehat{\mathbf{F}_{\mathbf{0}}}}$ en la Figura 5.3 como subgrafo inducido.

Demostración: La equivalencia de $i$ ) y ii) es consecuencia directa del Teorema 5.1.8. Para probar $i i$ ) implica $i i i)$, sea $G$ un grafo dualmente- $C P T$. Entonces existe un poset $\mathbf{P}$ dualmente- $C P T$ tal que $G=G_{\mathbf{P}}$. Asumamos, para llegar a una contradicción, que $G$ tiene como subgrafo inducido a $G_{\widehat{\mathbf{F}_{\mathbf{0}}}}$. Entonces $\mathbf{P}$ tiene un subposet $\mathbf{P}^{\prime}$ asociado a $\widehat{\mathbf{F}_{\mathbf{0}}}$. Por el Lema 5.1.7, debe ser $\mathbf{P}^{\prime}=\widehat{\mathbf{F}_{\mathbf{0}}}$ o $\mathbf{P}^{\prime}={\widehat{\mathbf{F}_{\mathbf{0}}}}^{d}$. En cualquier caso se contradice el Teorema 5.1.8, pues $\mathbf{P}$ es dualmente- $C P T$.

Para demostrar iii) implica $i$ ), con el objetivo de llegar a una contradicción, supongamos que $G$ no es fuertemente- $C P T$. Entonces existe un poset $\mathbf{P}$ que no es $C P T$ tal que $G=G_{\mathbf{P}}$. Por el Teorema 5.1.8, $\mathbf{P}$ tiene a $\widehat{\mathbf{F}_{\mathbf{0}}}$ o a ${\widehat{\mathbf{F}_{\mathbf{0}}}}^{d}$ como subposet. Luego $G_{\mathbf{P}}$ tiene a $G_{\widehat{\mathbf{F}_{\mathbf{0}}}}$ como subgrafo, contradiciendo $\left.i i i\right)$.

Notar que aunque el Teorema 5.1.5 provee una caracterización por subposets prohibidos de los posets split que son CPT, la misma no es suficiente para obtener una caracterización por subgrafos prohibidos de los grafos split que son CPT. El grafo $G_{\widehat{\mathbf{F}_{\mathbf{0}}}}$ es un grafo split $C P T$ aunque el poset $\widehat{\mathbf{F}_{\mathbf{0}}}$ es un subposet prohibido para los posets split $C P T$.

En otras palabras, para que $G_{\mathbf{P}}$ sea un grafo no $C P T$ es necesario que $\mathbf{P}$ sea un poset no $C P T$ y que además todo poset $\mathbf{P}^{\prime}$ asociado a $\mathbf{P}$ también sea un poset no $C P T$. 
El Lema 5.1.9 implica que los grafos $G_{\mathbf{R}}, G_{\mathbf{T}}, G_{\mathbf{U}}$ y $G_{\mathbf{V}}$ descriptos en la Figura 5.3 no son grafos $C P T$. En particular son subgrafos inducidos prohibidos de la clase de grafos split $C P T$. Conjeturamos que son los únicos.

Conjetura 5.2.2. Un grafo split $G$ es $C P T$ si y solamente si $G$ no tiene a $G_{\mathbf{R}}$, ni a $G_{\mathbf{T}}$, ni a $G_{\mathbf{U}}$, ni a $G_{\mathbf{V}}$ en la figura 5.3 como subgrafo inducido. 


\section{Capítulo 6}

\section{Posets y grafos $C P T$ en la clase $k$-tree}

En este capítulo estudiamos a los posets $k$-tree que admiten un modelo por contención de caminos en un árbol. Mostramos que en dicha clase la condición necesaria para ser poset $C P T$, el Lema 4.1.2, es también suficiente. En la Sección 6.1, consideraremos el caso de los posets 2-tree. Luego en la Sección 6.2, generalizaremos los resultados a posets $k$-tree para un entero positivo $k$ cualquiera. El objetivo de introducir una primera sección atendiendo el caso particular de los posets 2-tree es facilitar el entendimiento del caso general. El lector que así lo prefiriera puede comenzar directamente con la Sección 6.2. En esta sección, obtenemos también caracterizaciones por subposets prohibidos de las clases de posets $k$-tree $C P T, k$-tree fuertemente- $C P T$ y $k$-tree dualmente-CPT. En la última sección presentamos sendas caracterizaciones por subgrafos inducidos prohibidos de las clases de grafos $k$-tree fuertemente-CPT y $k$-tree dualmente-CPT.

Dado un entero positivo $k$, un grafo $G$ es $\boldsymbol{k}$-tree si puede ser construido de manera recursiva comenzando con un completo formado por $k$ vértices y luego, en cada paso, adicionando un vértice con exactamente $k$ vecinos los cuales inducen un completo [5]. 
Observar que los grafos 1-tree son los árboles, es decir los grafos conexos sin cíclos. Los grafos $k$-tree son grafos cordales. Si $G$ es un grafo $k$-tree que no se reduce a un completo de tamaño $k$, entonces todos los cliques de $G$ tienen tamaño $k+1$ y sus conjuntos separadores minimales son completos de tamaño $k$ [5].

Definición 6.0.3. Un poset $\mathbf{P}$ es $k$-tree si su grafo de comparabilidad $G_{\mathbf{P}}$ es un grafo $k$-tree.

Observación 6.0.4. Si $\mathbf{P}$ es $k$-tree entonces $k \leq h(\boldsymbol{P}) \leq k+1$. Más aun, si $h(\mathbf{P})=k$, entonces $\mathbf{P}$ es una cadena.

\subsection{Posets 2-tree}

Así como los grafos 2-tree pueden ser construidos mediante un proceso recursivo, los posets 2-tree también se construyen usando un proceso recursivo, el cual describimos a continuación.

Proceso constructivo 6.1.1. Sea $\mathbf{P}^{\prime}=\left(X^{\prime}, P^{\prime}\right)$ un poset. Dados dos elementos c y a de $X^{\prime}$, tales que $c<$ a en $\mathbf{P}^{\prime}$ y tales que satisfacen una y solo una de las siguientes condiciones

(i) $X^{\prime}=\{a, c\}$,

(m) $X^{\prime} \neq\{a, c\}$ y $D_{\mathbf{P}^{\prime}}(c)=\emptyset$ y $U_{\mathbf{P}^{\prime}}(a)=\emptyset$,

(u) $X^{\prime} \neq\{a, c\}$ y $D_{\mathbf{P}^{\prime}}(a)=\{c\}$,

(d) $X^{\prime} \neq\{a, c\}$ y $U_{\mathbf{P}^{\prime}}(c)=\{a\}$

se define un nuevo poset $\mathbf{P}=(X, P)$ en la forma

$$
X=X^{\prime} \cup\{z\} \text { con } z \notin X^{\prime}
$$


y $P$ la relación que se escribe de la siguiente manera, dependiendo de la situación de los elementos a y c, a saber:

$$
P=\left\{\begin{array}{lc}
P^{\prime} \cup\{(z, z),(c, z),(a, z)\} & \text { si }(\boldsymbol{i}) \\
P^{\prime} \cup\{(z, z),(c, z),(z, a)\} & \text { si }(\boldsymbol{m}) \\
P^{\prime} \cup\{(z, z),(c, z),(a, z)\} & \text { si }(\boldsymbol{u}) \\
P^{\prime} \cup\{(z, z),(z, a),(z, c)\} & \text { si }(\boldsymbol{d})
\end{array}\right.
$$

Es fácil ver que $P$ extiende a $P^{\prime}$, está bien definida y es una relación reflexiva, antisimétrica y transitiva, por lo cual $\left(X^{\prime}, P^{\prime}\right)$ es subposet de $(X, P)$.

Lema 6.1.2. Un poset $\mathbf{P}$ es 2 -tree si y solo si $\mathbf{P}$ se obtiene a partir de una cadena de 2 elementos aplicando una cantidad finita de veces el Proceso constructivo 6.1.1.

Demostración: Si $\mathbf{P}=(X, P)$ es un poset 2-tree, demostraremos por inducción sobre la cantidad de elementos en $X$ que $\mathbf{P}$ se obtiene, a partir de una cadena de 2 elementos, usando el Proceso constructivo 6.1.1.

Si $|X|=2$ o 3 entonces $\mathbf{P}$ es una cadena de dos o tres elementos, respectivamente, $\mathrm{y}$ la prueba es trivial.

Si $|X|>3$, como $G_{\mathbf{P}}$ es 2-tree existe un vértice $z \in X$ y un clique $\{x, y, u\}$ tal que $N(z)=\{x, y\}, u \neq z$ y $G^{\prime}=G_{\mathbf{P}}-\{z\}$ es 2-tree. Sea $\mathbf{P}^{\prime}=(X-\{z\}, P(X-\{z\}))$. Es claro que $\mathbf{P}^{\prime}$ es subposet de $\mathbf{P}$ y $G_{\mathbf{P}^{\prime}}=G^{\prime}$, entonces se tiene que $\mathbf{P}^{\prime}$ se puede construir usando el Proceso constructivo 6.1.1.

Como $x, y$ y $u$ forman una cadena maximal en $\mathbf{P}$, basta considerar los siguientes casos:

Caso I) Asumimos que $x<u<y$ en $\mathbf{P}$, entonces $D_{\mathbf{P}^{\prime}}(x)=\emptyset$ y $U_{\mathbf{P}^{\prime}}(y)=\emptyset$. Como $N(z)=\{x, y\}$ en $G_{\mathbf{P}}, z$ debe ser comparable con $x$ y con $y$ pero no con $u$, implicando que $x<z<y$ en $\mathbf{P}$. Por lo tanto, tomando $c=x$ y $a=y$, el poset $\mathbf{P}$ se obtiene a partir $\mathbf{P}^{\prime}$, usando el Proceso 6.1.1, agregando z según $(\mathbf{m})$. 
Caso II) Si $x<y<u$ en $\mathbf{P}$, ya que $z$ es comparable con $x$ y con $y$ pero no con $u$, tenemos que $x<y<z$ en $\mathbf{P}$. Veremos que $D_{\mathbf{P}^{\prime}}(y)=\{x\}$. Con el objetivo de llegar a una contradicción, supongamos que existe un elemento $h \neq x$ en $D_{\mathbf{P}^{\prime}}(y)$. Si $h$ fuese comparable con $x$ se tendría una cadena de longitud 4 formada por $\{h, x, y, z\}$; luego $x$ y $h$ deben ser incomparables, pero entonces en $G_{\mathbf{P}}$ hay un ciclo inducido por los vértices $\{u, h, z, x\}$, lo cual contradice el hecho que $G_{\mathbf{P}}$ es cordal. Luego, tomando $c=x$ y $a=y$, se obtiene el poset $\mathbf{P}$ a partir de $\mathbf{P}^{\prime}$, usando el Proceso 6.1.1, agregando $z$ según $(\mathbf{u})$.

Caso III) Si $u<x<y$ en $\mathbf{P}$, entonces, de forma análoga al caso anterior, el poset $\mathbf{P}$ se obtiene a partir de $\mathbf{P}^{\prime}$, usando el Proceso 6.1.1, agregando $z$ según $(\mathbf{d})$.

Para probar la implicación recíproca basta observar que en cada paso del proceso 6.1.1 el vértice $z$ que se agrega es comparable solamente con los elementos a y $c$, los cuales a su vez son comparables. Luego en el grafo de comparabilidad $G_{\mathbf{P}}$ el elemento $z$ es adyacente a los vértices $a$ y $c$, los cuales forman completo de tamaño 2 .
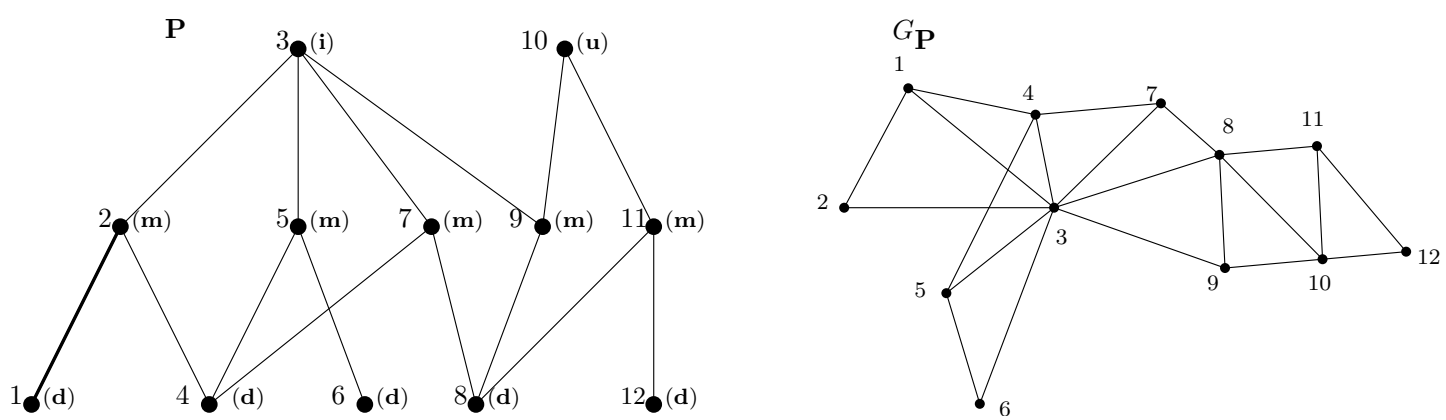

Figura 6.1: Diagrama de Hasse de un poset construido de acuerdo al Proceso constructivo 6.1.1. Los vértices están enumerados siguiendo el orden en que aparecen en el proceso iniciado por la cadena cuyo conjunto de elementos es $\{1,2\}$. Además, están etiquetados con $(\mathbf{i}),(\mathbf{m}),(\mathbf{d})$ o (u) según cómo hayan sido agregados.

En la Figura 6.1 podemos ver el diagrama de Hasse de un poset construido de acuerdo al Proceso 6.1.1, cuyo conjunto de elementos es $\{n \in \mathbb{N}: 1 \leq n \leq 12\}$. Por el Lema 
6.1.2, dicho poset es 2-tree. En la misma Figura se observa su grafo de comparabilidad. Resulta del Lema anterior que los vértices de un poset 2-tree $\mathbf{P}=(X, P)$ pueden ser totalmente ordenados $z_{1}, z_{2}, \ldots, z_{n}$ y etiquetados con $(\mathbf{i}),(\mathbf{m}),(\mathbf{u})$ o $(\mathbf{d})$, según el proceso constructivo descripto anteriormente; siendo $z_{1}<z_{2}$ los elementos de la cadena inicial y $z_{n}$ el último elemento agregado. Los vértices $z_{1}$ y $z_{2}$ se etiquetan con (d) y (m), respectivamente.

Definición 6.1.3. Un orden de los elementos de un poset 2-tree $\mathbf{P}$ obtenido según el Proceso Constructivo 6.1.1 será llamado orden constructivo de $\mathbf{P}$.

Los lemas que demostramos a continuación son necesarios para probar el resultado principal de esta sección, el Teorema 6.1.8. En este teorema probamos que la condición necesaria para ser poset CPT del Lema 4.1.2 es también suficiente para la clase de posets 2-tree.

Lema 6.1.4. Si $z_{1}, z_{2}, \ldots, z_{n}$ es un orden constructivo de $\mathbf{P}$, entonces (i) $z_{3}$ es el único elemento con etiqueta (i) y además $z_{1}<z_{2}<z_{3}$ y $z_{3}$ es maximal. (ii) Si $j>3$ entonces un elemento $z_{j}$ es maximal en $\mathbf{P}$ si y solo tiene etiqueta (u). (iii) Un elemento $z_{j}$ es minimal en $\mathbf{P}$ si y solo si tiene etiqueta $(\boldsymbol{d})$. (iv) Si $z_{j}$ tiene etiqueta (d) y $j>3$, entonces existe un único $i<j$ tal que $z_{i}$ es maximal en $\mathbf{P}$.

(v) Si $z_{j}$ tiene etiqueta $(\boldsymbol{m})$ y $j>3$, entonces existe un único $i<j$ tal que $z_{i}$ es maximal en $\mathbf{P}$.

Demostración: Los items $(i),(i v)$ y $(v)$ son inmediatos a partir de las condiciones del proceso constructivo.

Para probar (ii) observar que si $z$ es un maximal entonces, en el proceso de construcción recursivo, él no pudo ser agregado con etiqueta $(\mathbf{m})$ o $(\mathbf{d})$, ya que en estos casos $U(z) \neq \emptyset$. Por este motivo podemos asegurar que $z$ fue agregado con etiqueta (u). 
Recíprocamente, si en un paso de la construcción de $\mathbf{P}$ el elemento $z$ es agregado con etiqueta (u) entonces $z$ forma parte de la cadena $c<a<z$. Luego, por la Observación 6.0.4, $U(z)=\emptyset$. De manera similar se prueba (iii).

A continuación vamos a demostrar que si $\mathbf{P}$ es un poset 2-tree entonces puede ser construido de forma tal que si $z_{r}$ es el último maximal en ser agregado, entonces todo elemento que se agregó después que $z_{r}$ pertenece a su conjunto descendente.

Lema 6.1.5. Sea $\boldsymbol{P}=(X, P)$ un poset 2-tree. Existe un orden constructivo $z_{1}, z_{2}, \ldots, z_{n}$ de $\boldsymbol{P}=(X, P)$ tal que si $r=\operatorname{máx}\left\{i: z_{i}\right.$ es maximal en $\left.\boldsymbol{P}\right\}$ entonces $z_{i} \in D\left[z_{r}\right]$ para todo $i \geq r$.

Demostración: La demostración será por inducción sobre la cantidad de elementos de $\mathbf{P}$. Es fácil ver que el Lema es cierto si $|X| \leq 3$

Sea $z_{1}, z_{2}, \ldots, z_{n}$ un orden constructivo con $n>3$. Si $z_{n}$ es maximal el resultado es trivial.

Supongamos que $z_{n}$ no es un elemento maximal. Llamemos $a$ y $c$ a los dos únicos elementos de $\mathbf{P}$ comparables con $z_{n}$.

Como $\mathbf{P}-\left\{z_{n}\right\}$ es un poset 2-tree, por hipótesis inductiva existe un orden constructivo $z_{1}^{\prime}, z_{2}^{\prime}, \ldots, z_{n-1}^{\prime}$ de $\mathbf{P}^{\prime}=\mathbf{P}-\left\{z_{n}\right\}$ tal que si $z_{r}^{\prime}$ es el maximal que fue agregado último entonces $z_{i}^{\prime} \in D\left(z_{r}^{\prime}\right)$ para todo $i>r$. Observar que el conjunto ascendente y el conjunto descendente de cada elemento es independiente del orden constructivo. Indicaremos, respectivamente, mediante $D_{\mathbf{P}^{\prime}}(x)$ y $U_{\mathbf{P}^{\prime}}(x)$ a los conjuntos descendente y ascendente del elemento $x$ en $\mathbf{P}-\left\{z_{n}\right\}$. Sean $j_{a}$ y $j_{c}$ tales que $a=z_{j_{a}}^{\prime}$ y $c=z_{j_{c}}^{\prime}$. Si $z_{n} \in D\left(z_{r}^{\prime}\right)$ basta agregar $z_{n}$ en un último paso y el Lema es cierto.

Si $z_{n} \notin D\left(z_{r}^{\prime}\right)$ y no es ni maximal ni minimal de $\mathbf{P}$ entonces debe ocurrir que $D(c)=$ $U(a)=\emptyset$, luego $D_{\mathbf{P}^{\prime}}\left(z_{j_{c}}^{\prime}\right)=U_{\mathbf{P}^{\prime}}\left(z_{j_{a}}^{\prime}\right)=\emptyset$. Dado que $z_{n} \notin D\left(z_{r}^{\prime}\right)$ y $z_{n} \in D\left(z_{j_{a}}^{\prime}\right)$, debe ser $j_{a}<r$. Por otra parte, si $j_{c}>r$ entonces $j_{c}>3$ y $z_{j_{c}}^{\prime}<z_{r}^{\prime}$; luego, por Lema 6.1.4 
$(i v)$, debe ser $z_{r}^{\prime}=z_{j_{a}}^{\prime}$, lo cual contradice la asunción $z_{n} \notin D\left(z_{r}^{\prime}\right)$ ya que $z_{n} \in D\left(z_{j_{a}}^{\prime}\right)$. Resulta entonces que $j_{c}<r$, y así $z_{n}$ puede ser agregado inmediatamente antes que $z_{r}^{\prime}$, obteniendo un orden constructivo de $\mathbf{P}$ como se deseaba.

Finalmente, si $z_{n} \notin D\left(z_{r}^{\prime}\right)$ y $z_{n}$ es minimal, es decir tiene etiqueta (d), entonces $z_{n}<c<a$ en $\mathbf{P}$ y $U(c)=\{a\}$. Por lo tanto $z_{j_{c}}^{\prime}<z_{j_{a}}^{\prime}$ y $U_{\mathbf{P}^{\prime}}\left(z_{j_{c}}^{\prime}\right)=\left\{z_{j_{a}}^{\prime}\right\}$. Como $z_{n} \notin D\left(z_{r}^{\prime}\right)$, pero a la vez $z_{n} \in D\left(z_{j_{a}}^{\prime}\right)$, debe ser $j_{a}<r$ y además $j_{c}<r$. En efecto, si $j_{c}>r$ entonces $z_{j_{c}}^{\prime}<z_{r}^{\prime}$, y por ende $z_{n} \in D\left(z_{r}^{\prime}\right)$. Por lo tanto $z_{n}$ puede ser agregado inmediatamente antes que $z_{r}^{\prime}$, obteniendo un orden constructivo de $\mathbf{P}$ que satisface lo pedido.

Definición 6.1.6. Un orden de los elmentos de un poset $\mathbf{P}$ en las condiciones del Lema 6.1.5 será llamado buen orden constructivo de $\mathbf{P}$.

Lema 6.1.7. Sea $z_{1}, z_{2}, \ldots, z_{r-1}, z_{r}, z_{r+1}, \ldots, z_{n}$, con $r \geq 3$, un buen orden constructivo de un poset 2 -tree $\mathbf{P}$, donde $z_{r}$ satisface las condiciones del Lema anterior. Entonces,

- $\left\{z_{1}, z_{2}, \ldots, z_{r-1}\right\} \cap D\left(z_{r}\right)=\left\{z_{j_{1}}, z_{j_{2}}\right\}$, con $z_{j_{1}}<z_{j_{2}}<z_{r}$ en $\mathbf{P}$.

- Si $z_{h} \in\left\{z_{1}, z_{2}, \ldots, z_{r-1}\right\}-\left\{z_{j_{1}}, z_{j_{2}}\right\}$ y $j>r$ entonces $z_{h} \| z_{j}$.

Demostración: El primer item es trivial, los elementos $z_{j_{1}} \mathrm{y} z_{j_{2}}$ son los elementos $a$ y $c$ en el caso (u) del proceso constructivo.

Para probar el segundo item, y con el objetivo de llegar a una contradicción, asumimos que $z_{h} \perp z_{j}$ en $\mathbf{P}$.

Si $z_{h}$ es maximal en $\mathbf{P}$ ya que $z_{j}$ debe ser comparable con $z_{h}, z_{j}$ debe tener etiqueta $(\mathbf{m})$ o $(\mathbf{d})$. Entonces los maximales $z_{h}$ y $z_{r}$ son comparables con $z_{j}$ siendo $h<j \mathrm{y}$ $r<j$. Luego, ya que $j>3$, por la parte $(v)$ o $(i v)$ del Lema 6.1.4, respectivamente tenemos una contradicción. 
Si $z_{h}$ tiene etiqueta $(\mathbf{m})$, como $z_{j}$ se supone comparable con $z_{h}$ y no puede ser maximal por definición de $z_{r}$, entonces $z_{j}$ debe ser un elemento minimal de $\mathbf{P}$. Si asumimos que $h>3$, por $(v)$ del Lema 6.1.4 existe un único $k<h$ tal que $z_{h}<z_{k}$ y $z_{k}$ es maximal. Entonces $z_{j}<z_{h}<z_{k}$ en $\mathbf{P}$, lo que contradice (iv) del Lema 6.1.4. Si $h \leq 3$, se cumple que $h=2$ y $z_{2}<z_{3}$ en $\mathbf{P}$; si $z_{3} \neq z_{r}$, esto último implica que $z_{j}<z_{3}$ lo que contradice (iv) del Lema 6.1.4. Y si $z_{3}=z_{r}$ entonces $z_{h}=z_{2} \in D\left(z_{r}\right)$, lo que contradice nuestra asunción sobre el elemento $z_{h}$.

Si $z_{h}$ es minimal de $\mathbf{P}$, se tendría entonces que $z_{h}<z_{j}<z_{r}$. Esto último es una contradicción. Por lo tanto, concluimos que $z_{h} \| z_{j}$ en $\mathbf{P}$.

Teorema 6.1.8. Sea $\mathbf{P}=(X, P)$ un poset 2-tree. $\mathbf{P}$ admite un modelo $C P T$ si y solo si $D[z]$ es $C I$ para todo $z$ maximal de $\mathbf{P}$.

Demostración: La implicación directa vale en general como lo demuestra el Lema 4.1.2.

Para demostrar la proposición recíproca, primero asumamos que $\mathbf{P}$ no tiene falsos gemelos. Probaremos que si tenemos un poset $\mathbf{P}=(X, P)$ sin falsos gemelos y 2-tree tal que $\mathbf{P}(D[z])$ es $C I$ para todo $z$ elemento maximal de $\mathbf{P}$, entonces $\mathbf{P}$ admite un modelo $C P T$ en el cual ningún camino se reduce a un vértice y ningún par de caminos tiene un extremo coincidente. Procederemos por inducción sobre $|X|$.

Si $|X|=2$ o 3 entonces $\mathbf{P}$ es una cadena y la demostración es trivial.

Sea $|X|=n>3$. Por el Lema 6.1.5 existe un buen orden constructivo de $\mathbf{P}$ $z_{1}, z_{2}, \ldots z_{r}, z_{r+1}, \ldots, z_{n}$. Sea $z_{r}$ el maximal que fue agregado último en este proceso constructivo. Luego $z_{i} \in D\left[z_{r}\right]$ para todo $i \geq r+1$.

Llamemos $\mathbf{P}^{\prime}$ al subposet $\mathbf{P}\left(X^{\prime}\right)$ donde $X^{\prime}=\left\{z_{1}, z_{2}, \ldots, z_{r-1}\right\}$. Como $\mathbf{P}^{\prime}$ es 2-tree, entonces, por hipótesis inductiva, existe un modelo $C P T R_{\mathbf{P}^{\prime}}=\left(W_{z_{i}}^{\prime}\right)_{z_{i} \in X^{\prime}}$ sobre un 

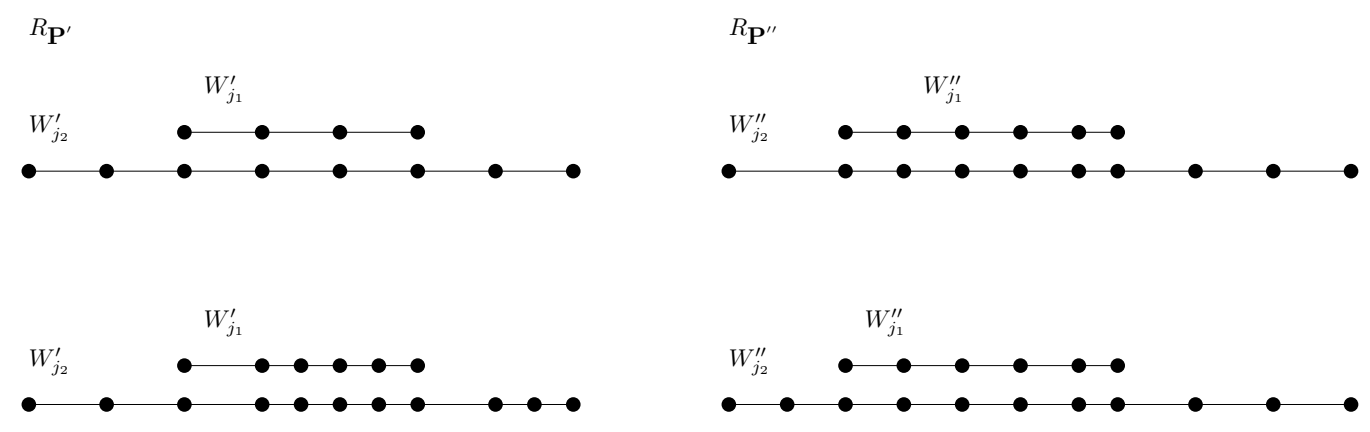

Figura 6.2: En la parte superior, a derecha e izquierda, vemos a los caminos que representan los elementos $j_{1}$ y $j_{2}$ en los modelos $R_{\mathbf{P}^{\prime}}$ y $R_{\mathbf{P}^{\prime \prime}}$, respectivamente. En la parte inferior, vemos el resultado de subdividir aristas en los modelos $R_{\mathbf{P}^{\prime}}$ y $R_{\mathbf{P}^{\prime \prime}}$ para lograr que en ambos modelos: $W_{j_{1}}^{\prime}$ y $W_{j_{1}}^{\prime \prime}$ tengan la misma longitud, $W_{j_{2}}^{\prime}$ y $W_{j_{2}}^{\prime \prime}$ tengan igual longitud, y la posición relativa de $W_{j_{1}}^{\prime}$ dentro de $W_{j_{2}}^{\prime}$ sea igual a la posición relativa de $W_{j_{1}}^{\prime \prime}$ dentro de $W_{j_{2}}^{\prime \prime}$.

árbol huésped $T^{\prime}$, en el cual ningún camino se reduce a un punto y ningún par de caminos tiene un extremo coincidente.

Por otro lado, sea $X^{\prime \prime}=D_{\mathbf{P}}\left[z_{r}\right]$. Por hipótesis $\mathbf{P}^{\prime \prime}=\mathbf{P}\left(X^{\prime \prime}\right)$ es $C I$. Luego es posible obtener un modelo $C I R_{\mathbf{P}^{\prime \prime}}=\left(W_{z_{i}}^{\prime \prime}\right)_{z_{i} \in D\left[z_{r}\right]}$, donde ningún camino se reduce a un vértice y ningún par de caminos tiene un extremo coincidente [6] .

En lo que sigue, reacomodaremos los modelos $R_{\mathbf{P}^{\prime}}$ y $R_{\mathbf{P}^{\prime \prime}}$ de manera tal que su superposición nos permita obtener un modelo $C P T$ de $\mathbf{P}$.

Por el primer item del Lema 6.1.7, se tiene que $X^{\prime} \cap X^{\prime \prime}=\left\{z_{j_{1}}, z_{j_{2}}\right\}$ con $j_{1}, j_{2}<r$ y $z_{j_{1}}<z_{j_{2}}<z_{r}$ en $\mathbf{P}$. Luego los vértices $z_{j_{i}}$ están representados tanto en el modelo $R_{\mathbf{P}^{\prime}}$ como en $R_{\mathrm{P}^{\prime \prime}}$.

En ambos modelos cada camino tienen al menos dos vértices, por lo que es posible subdividir una arista para aumentar la longitud de un camino cualquiera. Es claro que subdividiendo aristas donde sea necesario, en el modelo $R_{\mathbf{P}^{\prime}}$ o en el modelo de $R_{\mathbf{P}^{\prime \prime}}$, se puede lograr que $W_{j_{1}}^{\prime}$ y $W_{j_{1}}^{\prime \prime}$ tengan la misma longitud, que $W_{j_{2}}^{\prime}$ y $W_{j_{2}}^{\prime \prime}$ tengan igual longitud, y que la posición relativa de $W_{j_{1}}^{\prime}$ dentro de $W_{j_{2}}^{\prime}$ sea igual a la posición relativa de $W_{j_{1}}^{\prime \prime}$ dentro de $W_{j_{2}}^{\prime \prime}$ (ver Figura 6.2). 


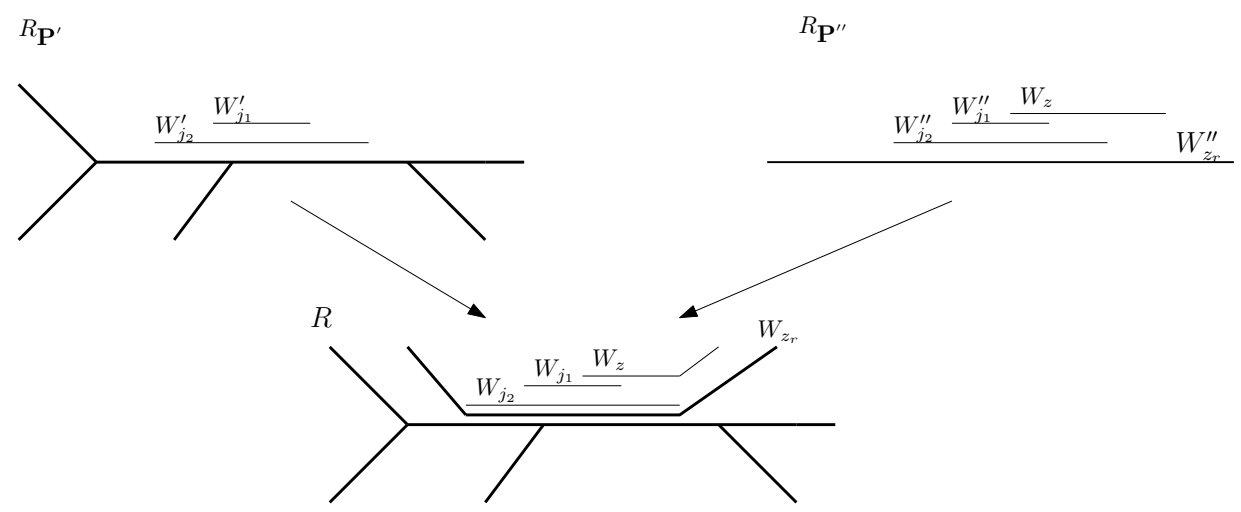

Figura 6.3: Modelo $R$ que resulta de superponer convenientemente los modelos $R_{\mathbf{P}^{\prime}}$ y $R_{\mathbf{P}^{\prime \prime}}$.

Sea $R$ el modelo por contención de caminos que se obtiene uniendo los modelos $R^{\prime}$ y $R^{\prime \prime}$, de forma que $W_{z_{j_{1}}}^{\prime}$ se identifica con $W_{z_{j_{1}}}^{\prime \prime}$ y $W_{z_{j_{2}}}^{\prime}$ se identifica con $W_{z_{j_{2}}}^{\prime \prime}$. Y la parte de $W_{z_{r}}^{\prime \prime}$ que no está cubierta por $W_{z_{j_{2}}}^{\prime \prime}$ se representa en $T^{\prime}$ agregando 2 nuevas ramas en cada uno de los extremos. Luego, $W_{z_{r}}^{\prime \prime} \cap T^{\prime}=W_{j_{2}}^{\prime}=W_{j_{2}}^{\prime \prime}$, como se muestra en la Figura 6.3.

En el modelo $R$ llamamos $W_{z}$ al camino correspondiente al elemento $z$. Es claro que $W_{z_{i}}=W_{z_{i}}^{\prime}$ si $i<r$ y $W_{z_{i}}=W_{z_{i}}^{\prime \prime}$ si $i \geq r$.

A continuación probaremos que $R$ es un modelo $C P T$ de $\mathbf{P}$.

Primero veamos que si $z_{i}<z_{j}$ en $\mathbf{P}$ entonces $W_{z_{i}} \subset W_{z_{j}}$.

Si $z_{i}, z_{j} \in X^{\prime}$ o $z_{i}, z_{j} \in X^{\prime \prime}$ entonces $z_{i}<z_{j}$ en $\mathbf{P}^{\prime}$ o $\mathbf{P}^{\prime \prime}$, respectivamente, lo cual implica que $W_{z_{i}}^{\prime} \subset W_{z_{j}}^{\prime}$ o $W_{z_{i}}^{\prime \prime} \subset W_{z_{j}}^{\prime \prime}$. Por lo tanto $W_{z_{i}} \subset W_{z_{j}}$.

Si $z_{i} \in X^{\prime}$ y $z_{j} \in X^{\prime \prime}$, entonces $z_{i}<z_{j}<z_{r}$ en $\mathbf{P}$; luego $z_{i} \in X^{\prime} \cap X^{\prime \prime}=\left\{z_{j_{1}}, z_{j_{2}}\right\}$. Por lo tanto, $z_{i}<z_{j}$ en $\mathbf{P}^{\prime \prime}$ y como vimos previamente se tiene que $W_{z_{i}} \subset W_{z_{j}}$.

Si $z_{i} \in X^{\prime \prime}$ y $z_{j} \in X^{\prime}$, entonces probaremos que $z_{j} \in X^{\prime \prime} \cap X^{\prime}$. En efecto, si $z_{j} \notin X^{\prime \prime}$ entonces por el Lema 6.1.7 se tiene que $z_{i} \| z_{j}$ lo cual contradice nuestra asunción. Por lo tanto $z_{j} \in X^{\prime} \cap X^{\prime \prime}$, y así se tiene que $z_{i}<z_{j}$ en $\mathbf{P}^{\prime \prime}$.

Ahora resta probar que si $W_{z_{i}}$ y $W_{z_{j}}$ son caminos pertenecientes a $R$ tales que $W_{z_{i}} \subset$ $W_{z_{j}}$ entonces $z_{i}<z_{j}$ en $\mathbf{P}$. 
Es claro que si ambos caminos pertenecen a $R_{\mathbf{P}^{\prime}}$ entonces $z_{i}<z_{j}$ en $\mathbf{P}^{\prime}$, lo que implica que $z_{i}<z_{j}$ en $\mathbf{P}$. Análogamente se prueba que si ambos caminos pertenecen a $R_{\mathbf{P}^{\prime \prime}}$ entonces $z_{i}<z_{j}$ en $\mathbf{P}$.

Si $W_{z_{i}} \in R_{\mathbf{P}^{\prime}}, W_{z_{j}} \in R_{\mathbf{P}^{\prime \prime}}$ y $W_{z_{i}} \subset W_{z_{j}}$, como también sabemos que $W_{z_{j}} \subset W_{z_{r}}$, se concluye que $W_{z_{i}} \subset W_{z_{r}}$. Esto a su vez implica que $W_{z_{i}} \subseteq W_{z_{j_{2}}}$. Estos dos últimos conjuntos pertenecen a $R_{\mathbf{P}^{\prime}}$, entonces la inclusión implica $z_{i} \leq z_{j_{2}}$. Además $W_{z_{j_{2}}}$ y $W_{r}$ están en $R_{\mathbf{P}^{\prime \prime}}$, por lo que $W_{z_{j_{2}}} \subset W_{z_{r}}$, entonces $z_{j_{2}}<z_{r}$. Por transitividad $z_{i}<z_{r}$. Por lo tanto $z_{i} \in X^{\prime \prime}$ y $W_{z_{i}} \in R_{\mathbf{P}^{\prime \prime}}$, además sabemos que $W_{z_{j}} \subset R_{\mathbf{P}^{\prime \prime}}$. Por ende $W_{z_{i}} \subset W_{z_{j}}$ implica que $z_{i}<z_{j}$.

Si $W_{z_{i}} \in R_{\mathbf{P}^{\prime \prime}}$ y $W_{z_{j}} \in R_{\mathbf{P}^{\prime}}$, consideramos dos casos: $z_{j} \in X^{\prime} \cap X^{\prime \prime}$ o no.

Si $z_{j} \in X^{\prime} \cap X^{\prime \prime}$, entonces $W_{z_{j}} \in R_{\mathbf{P}^{\prime \prime}}$. Así, se tiene que $z_{i}<z_{j}$ en $\mathbf{P}^{\prime \prime}$.

Consideremos el caso $z_{j} \notin X^{\prime} \cap X^{\prime \prime}$, si $z_{i} \in X^{\prime}$, entonces $W_{z_{i}}$ también está en $R_{\mathbf{P}^{\prime}}$. Entonces $W_{z_{i}} \subset W_{z_{j}}$ en $R_{\mathbf{P}^{\prime}}$ implica $z_{i}<z_{j}$. Si $z_{i} \notin X^{\prime}$ entonces $z_{i}=z_{r}$ o $i>r$. Si $z_{i}=z_{r}$, es absurdo porque todos los vértices de $W_{z_{r}}$ no están en el árbol huésped de $R_{\mathbf{P}^{\prime}}$ (lo más que llegan a compartir es $W_{z_{j_{2}}}$ ).

Si $z_{i} \neq z_{r}$, con $i>r, W_{z_{i}} \subseteq W_{z_{j}}$ implica $W_{z_{i}} \subseteq W_{z_{j_{2}}}$. Entonces $W_{z_{i}} \subset W_{z_{j_{2}}} \subset W_{z_{r}}$ en $R_{\mathbf{P}^{\prime \prime}}$ implica $z_{i}<z_{j_{2}}<z_{r}$. Entonces $z_{i}$ es un minimal con etiqueta (d). Por lo tanto, cuando $z_{i}$ fue agregado solo miraba a $z_{j_{2}}$ y $z_{r}$ y, por etiqueta $(\mathbf{d}), U\left(z_{j_{2}}\right)=z_{r}$ (en ese estadio de la construcción). Entonces, en ese momento $z_{j_{2}}$ se relacionaba con un único maximal que es $z_{r}$. Si $z_{r}$ es el único maximal, entonces $X^{\prime \prime}=X$, y $W_{z_{i}}, W_{z_{j}} \in R_{\mathbf{P}^{\prime \prime}}$. Si $z_{r}$ no fuera el único maximal, por construcción, al momento de ser agregado, $z_{j_{1}} \mathrm{y}$ $z_{j_{2}}$ estaba en una cadena junto a otro maximal $z_{k}$. Eso es absurdo porque contradice la etiqueta $(\mathbf{d})$ de $z_{i}$.

Concluimos que $R$ es un modelo $C P T$ del poset $\mathbf{P}$ en el cual todos los caminos tienen longitud mayor o igual a uno.

Podría ocurrir que existan caminos que tengan un extremo coincidente en $R$. Como asumimos que $\mathbf{P}$ no tiene falsos gemelos, por Lema 4.1.13 podemos asegurar que existe 
un modelo $C P T$ de $\mathbf{P}$ en el cual ningún par de caminos tiene un extremo coincidente. Si $\mathbf{P}$ tiene falsos gemelos, por cada clase de equivalencia de falsos gemelos podemos dejar un representante. Así, por lo demostrado previamente, tenemos un nuevo poset $C P T \mathbf{P}_{\mathbf{1}}$, el cual es 2-tree y no tiene falsos gemelos. Luego, por la Observación 4.1.6, $\mathbf{P}$ es $C P T$.

\subsection{Posets $k$-tree}

En esta sección generalizaremos los resultados demostrados para los posets 2-tree. Primero veremos, que así como los grafos $k$-tree se construyen recursivamente, los posets $k$-tree también admiten una construcción recursiva.

Proceso constructivo 6.2.1. Sea $\boldsymbol{P}^{\prime}=\left(X^{\prime}, P^{\prime}\right)$ un poset y $c<$ a elementos tales que se satisface una y solo una de las siguientes condiciones:

(i) $\boldsymbol{P}^{\prime}$ es un cadena de $k$ elementos $y X^{\prime}=D_{\boldsymbol{P}^{\prime}}[a]$.

(m) $X^{\prime} \neq D_{\boldsymbol{P}^{\prime}}[a], U_{\boldsymbol{P}^{\prime}}[a]$ y $D_{\boldsymbol{P}^{\prime}}[c]$ inducen una cadena $y|U[a]|+|D[c]|=k$.

(u) $X^{\prime} \neq D_{\boldsymbol{P}^{\prime}}[a]$, y además $D_{\boldsymbol{P}^{\prime}}(c)=\emptyset, D_{\boldsymbol{P}^{\prime}}[a]$ induce una cadena $y\left|D_{\boldsymbol{P}^{\prime}}[a]\right|=k$

(d) $X^{\prime} \neq U_{\boldsymbol{P}^{\prime}}[c]$, y además $U_{\boldsymbol{P}^{\prime}}(a)=\emptyset, U_{\boldsymbol{P}^{\prime}}[c]$ induce una cadena $y\left|U_{\boldsymbol{P}^{\prime}}[c]\right|=k$.

se define un nuevo poset $\mathbf{P}=(X, P)$ en la forma

$$
X=X^{\prime} \cup\{z\} \text { con } z \notin X^{\prime}
$$

y $P$ la relación que se escribe de la siguiente manera, dependiendo de la situación de los elementos a y c, a saber: 


$$
P= \begin{cases}P^{\prime} \cup\{(z, z)\} \cup\left\{(x, z): x \in X^{\prime}\right\} & \text { si }(\boldsymbol{i}) \\ P^{\prime} \cup\{(z, z)\} \cup\left\{(x, z): x \in D_{\boldsymbol{P}^{\prime}}[c]\right\} \cup\left\{(z, x): x \in U_{\boldsymbol{P}^{\prime}}[a]\right\} & \text { si }(\boldsymbol{m}) \\ P^{\prime} \cup\{(z, z)\} \cup\left\{(x, z): x \in D_{\boldsymbol{P}^{\prime}}[a]\right\} & \text { si }(\boldsymbol{u}) \\ P^{\prime} \cup\{(z, z)\} \cup\left\{(z, x): x \in U_{\boldsymbol{P}^{\prime}}[c]\right\} & \text { si }(\boldsymbol{d})\end{cases}
$$

Es fácil ver que $P$ está bien definida y es una relación reflexiva, antisimétrica y transitiva, por lo que $\left(X^{\prime}, P^{\prime}\right)$ es subposet de $(X, P)$.

Lema 6.2.2. Un poset $\mathbf{P}$ es $k$-tree si y solo si $\mathbf{P}$ puede obtenerse a partir de una cadena de $k$ elementos aplicando una cantidad finita de veces el Proceso constructivo 6.2.1.

Demostración: Sea $\mathbf{P}=(X, P)$ un poset $k$-tree. Demostraremos por inducción sobre $|X|$ que $\mathbf{P}$ puede ser construido usando el Proceso constructivo 6.2.1.

Si $|X|=k$ o $k+1$ el grafo $G_{\mathbf{P}}$ es completo y entonces es $k$-tree; luego $\mathbf{P}$ se puede construir usando el Proceso constructivo 6.2.1, pues es una cadena de $k$ o $k+1$ elementos, respectivamente.

Si $|X|>k+1$, como $G_{\mathbf{P}}$ es $k$-tree, existe un vértice $z \in X$ y un clique $\left\{z_{1}, z_{2}, \ldots, z_{k}, u\right\}$ tal que $N(z)=\left\{z_{1}, z_{2}, \ldots, z_{k}\right\}, z \neq u$ y $G^{\prime}=G_{\mathbf{P}}-\{z\}$ es $k$-tree. Si $\mathbf{P}^{\prime}=(X-$ $\{z\}, P(X-\{z\}))$, entonces $\mathbf{P}^{\prime}$ se obtiene usando el proceso 6.2.1 y $G_{\mathbf{P}^{\prime}}=G^{\prime}$. Para demostrar que $\mathbf{P}$ puede ser construido usando el proceso 6.2.1 basta considerar los siguientes tres casos:

Caso I) Si $u$ es maximal en $\mathbf{P}^{\prime}$, entonces él es último en la cadena inducida por $\left\{z_{1}, z_{2}, \ldots, z_{k}, u\right\}$. Como $z$ es comparable con $z_{1}, z_{2}, \ldots, z_{k}$ pero no con $u$, entonces debe ser $z_{i}<z$ en $\mathbf{P}$ para $1 \leq i \leq k$. Esto implica que $z$ es maximal en $\mathbf{P}$. Sea $a \in\left\{z_{1}, z_{2}, \ldots, z_{k}\right\}$ tal que $a<: u$ y sea $c$ el primer elemento de la cadena inducida por $D_{\mathbf{P}^{\prime}}[a]$. En lo que sigue demostraremos que $D_{\mathbf{P}^{\prime}}[a]$ induce una cadena y $\left|D_{\mathbf{P}^{\prime}}[a]\right|=k$. Para demostrar que $D_{\mathbf{P}^{\prime}}[a]$ induce una cadena, asumamos lo contrario. Luego debe 
existir elementos $h$ y $z_{j_{0}}$ en $D_{\mathbf{P}^{\prime}}(a)$ tales que $h \| z_{j_{0}}$, pero entonces en $G_{\mathbf{P}}$ hay un ciclo inducido por el conjunto de vértices $\left\{u, z, h, z_{j_{0}}\right\}$, lo cual contradice el hecho que $G_{\mathbf{P}}$ es cordal. Si $\left|D_{\mathbf{P}^{\prime}}[a]\right|>k$, entonces $\left|D_{\mathbf{P}^{\prime}}[u]\right|>k+1$, luego en $\mathbf{P}^{\prime}$ hay una cadena de longitud $k+2$ lo que contradice que $\mathbf{P}^{\prime}$ es $k$-tree. Así podemos afirmar que $\left|D_{\mathbf{P}^{\prime}}[a]\right|=k$ y que $D_{\mathbf{P}^{\prime}}[a]$ induce una cadena. Luego a y $c$ satisfacen la condición $(\mathbf{u})$ del Proceso constructivo 6.2.1. Es decir, $\mathbf{P}$ se obtiene a partir $\mathbf{P}^{\prime}$ agregando $z$ según $(\mathbf{u})$.

Caso II) Si $u$ es minimal en $\mathbf{P}$ la demostración es análoga al Caso $\mathbf{I})$, resultando que $z$ se agrega usando $(\mathbf{d})$.

Caso III) Finalmente, supongamos que existen $a, c \in\left\{z_{1}, z_{2}, \ldots, z_{k}\right\}$ tales que $c<$ : $u<: a$ en $\mathbf{P}^{\prime}$. Probaremos que $z$ puede agregarse usando $(\mathbf{m})$.

Como $z$ es comparable con $c$ y $a$ pero no con $u$, se deduce que $c<z<a$ en $\mathbf{P}$. Los conjuntos $D_{\mathbf{P}^{\prime}}[c]$ y $U_{\mathbf{P}^{\prime}}[a]$ inducen una cadena cada uno, pues si alguno de estos conjuntos no indujera una cadena, entonces el elemento $z$ sería comparable en $\mathbf{P}^{\prime}$ con al menos $k+1$ elementos, lo cual es absurdo. Luego la cadena inducida por los elementos de $D_{\mathbf{P}^{\prime}}[c] \cup U_{\mathbf{P}^{\prime}}[a]$ es de tamaño $k$. Por lo tanto, el poset $\mathbf{P}$ se obtiene a partir $\mathbf{P}^{\prime}$ agregando $z$ según $(\mathbf{m})$.

Para probar la implicación recíproca basta observar que el vértice $z$ que se agrega en cada paso es comparable solo con los elementos de una cadena de tamaño $k$, siendo entonces $z$ adyacente solamente a los $k$ vértices de un completo en el grafo de comparabilidad.

Resulta del Lema anterior que los elementos de un poset $k$-tree $\mathbf{P}=(X, P)$ pueden ser totalmente ordenados $z_{1}, z_{2}, \ldots, z_{n}$ y etiquetados con $(\mathbf{i})(\mathbf{m}),(\mathbf{u})$ o $(\mathbf{d})$, según el proceso constructivo descripto anteriormente. Establecemos que $z_{1}, z_{2}, \ldots, z_{k}$ son los elementos de la cadena inicial, con $z_{1}<z_{2}<\ldots<z_{k}$ en $\mathbf{P}$. Llamamos $z_{n}$ al último elemento agregado. El elemento $z_{1}$ se etiqueta con (d) y los restantes elementos de la 
cadena inicial $z_{2}, z_{3}, \ldots, z_{k}$ son etiquetados con $(\mathbf{m})$.

Definición 6.2.3. Un orden de los elementos de un poset $k$-tree $\mathbf{P}$ obtenido según el Proceso Constructivo 6.2.1 será llamado orden constructivo de $\mathbf{P}$.

Los lemas que demostramos a continuación son necesarios para probar el resultado principal de esta sección, el Teorema 6.2.8. En este teorema probamos que la condición necesaria para ser poset $C P T$, Lema 4.1.2, es también suficiente para la clase de posets $k$-tree.

Lema 6.2.4. Si $z_{1}, z_{2}, \ldots, z_{k} \ldots, z_{n}$ es un orden de los elementos de un poset $\mathbf{P}$ según el proceso de construcción, entonces

(i) $z_{k+1}$ es el único elemento maximal de $\mathbf{P}$ con etiqueta $(\boldsymbol{i})$.

(ii) Si $j>k+1$ entonces un elemento $z_{j}$ es maximal de $\mathbf{P}$ si y solo si tiene etiqueta $(\boldsymbol{u})$.

(iii) Un elemento $z_{j}$ es minimal de $\mathbf{P}$ si y solo si tiene etiqueta (d).

(iv) Si $z_{j}$ tiene etiqueta (d) y $j>1$, entonces existe un único $i<j$ tal que $z_{i}$ es maximal $y z_{j}<z_{i}$ en $\mathbf{P}$.

(v) Si $z_{j}$ tiene etiqueta $(\boldsymbol{m})$ y $j>k+1$, entonces existe un único $i<j$ tal que $z_{i}$ es maximal $y z_{j}<z_{i}$ en $\mathbf{P}$.

\section{Demostración:}

Los items (i) , (iv) y (v) son inmediatos a partir de las condiciones del proceso constructivo.

Para probar (ii) observar que si $z$ es un maximal entonces, en el proceso de construcción recursivo, él no pudo ser agregado con etiqueta $(\mathbf{m})$ o $(\mathbf{d})$, ya que en estos casos $U(z) \neq \emptyset$. Por este motivo podemos asegurar que $z$ fue agregado con etiqueta (u). Recíprocamente, si en un paso de la construcción de $\mathbf{P}$ el elemento $z$ es agregado con etiqueta $(\mathbf{u})$ entonces $z$ es el eslabon superior de una cadena formada por $k+1$ elementos. Luego, por la Observación 6.0.4, $U(z)=\emptyset$. 
De manera similar se prueba (iii).

A continuación demostraremos que un poset $k$-tree $\mathbf{P}$ puede ser construido de forma tal que si $z_{r}$ es el último maximal en ser agregado, entonces todo elemento agregado después que $z_{r}$ pertenece a su conjunto descendente.

Lema 6.2.5. Sea $\mathbf{P}=(X, P)$ un poset $k$-tree. Existe un orden de construcción, $z_{1}, z_{2}, \ldots, z_{n}$, de $\mathbf{P}=(X, P)$ tal que si $r=\max \left\{i: z_{i}\right.$ es maximal en $\left.\mathbf{P}\right\}$, entonces $z_{i} \in D\left[z_{r}\right]$ para todo $i \geq r$.

Demostración: La demostración será por inducción sobre la cantidad de vértices de P. Es fácil ver que el Lema es cierto si $|X|=k$.

Sea $z_{1}, z_{2}, \ldots, z_{n}$ un orden de construcción de $\mathbf{P}$ con $n>k$. Si $z_{n}$ es maximal el resultado es trivial.

Asumamos que $z_{n}$ no es un elemento maximal entonces, por (i) y (ii) del Lema 6.2.4, tiene etiqueta $(\mathbf{m})$ o $(\mathbf{d})$. En cualquier caso $z_{n}$ es comparable solo con $k$ elementos de $X$ los cuales inducen una cadena en $\mathbf{P}$.

Sea $z_{1}^{\prime}, z_{2}^{\prime}, \ldots, z_{n-1}^{\prime}$, un orden constructivo de $\mathbf{P}^{\prime}=\mathbf{P}-\left\{z_{n}\right\}$ tal que si $z_{r}^{\prime}$ es el maximal que fue agregado último entonces $z_{i}^{\prime} \in D\left(z_{r}^{\prime}\right)$ para todo $i>r$.

Si $z_{n} \in D\left(z_{r}^{\prime}\right)$ basta agregar $z_{n}$ en un último paso y el Lema es cierto.

Si $z_{n} \notin D\left(z_{r}^{\prime}\right)$ y tiene etiqueta $(\mathbf{m})$ entonces, según el proceso de construcción, existen $c$ y $a$ tales que $c<: z_{n}<: a$ en $\mathbf{P}$, los conjuntos $D[c]$ y $U[a]$ inducen una cadena y $|D[c]|+|U[a]|=k$. Sean $j_{a}$ y $j_{c}$ los subíndices de $a$ y $c$ en el orden de construcción de $\mathbf{P}^{\prime}$, es decir, $a=z_{j_{a}}^{\prime}$ y $c=z_{j_{c}}^{\prime}$. Debido a que $z_{r}^{\prime}$ es el último maximal en $\mathbf{P}^{\prime}$ se tiene que $j_{a}<r$. En efecto, si $r=j_{a}$ estamos en el caso anterior y si $r<j_{a}$ entonces $z_{j_{a}}^{\prime}<z_{r}^{\prime}$ en $\mathbf{P}$ lo cual implica que $z_{n} \in D\left(z_{r}^{\prime}\right)$ y esto es una contradicción. También se cumple que $j_{c}<r$. Para demostralo, asumamos que $r<j_{c}$, lo cual implica que $z_{j_{c}}^{\prime}<z_{r}^{\prime}$ en P. Si $z_{j_{a}}^{\prime}$ es maximal, entonces $z_{j_{c}}^{\prime}<z_{j_{a}}^{\prime}$ con $j_{a}<r$, lo que contradice $(v)$ del Lema 
6.2.4. Si $z_{j_{a}}^{\prime}$ no es maximal, entonces existe un único $j_{0}<j_{a}$ tal que $z_{j_{a}}^{\prime}<z_{j_{0}}^{\prime}$ y $z_{j_{0}}^{\prime}$ es maximal en $\mathbf{P}$. Como $z_{n}<z_{j_{a}}^{\prime}$ entonces $r \neq j_{0}$. Además, $z_{j_{c}}^{\prime}<z_{j_{a}}^{\prime}<z_{j_{0}}^{\prime}$ y $z_{j_{c}}^{\prime}<z_{r}^{\prime}$ lo que contradice $(i v)$ o $(v)$ del Lema 6.2.4.

Concluimos que $j_{a}<r$ y $j_{c}<r$, por lo cual $z_{n}$ puede ser agregado inmediatamente antes que $z_{r}^{\prime}$.

Finalmente, si $z_{n} \notin D\left(z_{r}^{\prime}\right)$ y tiene etiqueta $(\mathbf{d})$, entonces existen dos elementos $c$ y $a$ tales que $c<a$ en $\mathbf{P}, U(a)=\emptyset, U[c]$ induce una cadena, $|U[c]|=k$ y $z_{n}<c<a$ en $\mathbf{P}$. Nuevamente tomamos a $j_{a}$ y $j_{c}$ como los subíndices de $a$ y $c$ en orden de construcción de $\mathbf{P}^{\prime}$. Luego, como a es maximal en $\mathbf{P}$ se tiene que $j_{a}<r$. Además debe ser $j_{c}<r$, pues caso contrario se tendría que $c<z_{r}^{\prime}$ y así $z_{n} \in D\left(z_{r}^{\prime}\right)$ lo cual es una contradicción. Resulta que $z_{n}$ puede ser agregado inmediatamente antes que $z_{r}^{\prime}$, obteniéndose así un orden constructivo de $\mathbf{P}$ que satisface lo pedido.

Definición 6.2.6. Un orden de los elementos de un poset $\mathbf{P}$ en las condiciones del Lema 6.2.5 será llamado buen orden constructivo de $\mathbf{P}$.

Lema 6.2.7. Sea $z_{1}, z_{2}, \ldots, z_{r-1}, z_{r}, z_{r+1}, \ldots, z_{n}$ un buen orden constructivo de un poset $k$-tree $\mathbf{P}$, donde $z_{r}$ satisface las condiciones del Lema anterior. Entonces,

- $\left\{z_{1}, z_{2}, \ldots, z_{r-1}\right\} \cap D\left(z_{r}\right)=\left\{z_{j_{1}}, z_{j_{2}}, \ldots, z_{j_{k-1}}, z_{j_{k}}\right\}$, con $z_{j_{1}}<z_{j_{2}}<\ldots<$ $z_{j_{k-1}}<z_{j_{k}}$ en $\mathbf{P}$.

- Si $z_{h} \in\left(\left\{z_{1}, z_{2}, \ldots, z_{r-1}\right\}-\left\{z_{j_{1}}, z_{j_{2}}, \ldots, z_{j_{k}}\right\}\right)$ y $j>r$ entonces $z_{h} \| z_{j}$.

Demostración: El primer ítem es trivial, ya que los elementos $z_{j_{1}}, z_{j_{2}}, \ldots, z_{j_{k-1}}$ y $z_{j_{k}}$ forman la cadena del caso (u) del proceso constructivo.

Por el primer ítem es claro que $z_{h} \| z_{r}$. Para probar el segundo ítem, y con el objetivo de llegar a una contradicción, asumamos que $z_{h} \perp z_{j}$ en $\mathbf{P}$ con $j \neq r$. Si $z_{h}$ es maximal de $\mathbf{P}$ entonces, como $z_{j}$ es comparable con $z_{h}, z_{j}<z_{h}$ en $\mathbf{P}$. Además, $z_{j}$ puede tener 
etiquetas (m) o (d). Por otro lado, sabemos que $z_{j}<z_{r}$ en $\mathbf{P}$ con $h<r<j$; luego por $(i v)$ o $(v)$ del Lema 6.2.4 se llega a una contradicción.

Si $z_{h}$ tiene etiqueta $(\mathbf{m})$, ya que $z_{j}<z_{r} z_{j}$ puede tener etiqueta $(\mathbf{m})$ o $(\mathbf{d})$. Asumamos que $z_{j}$ tiene etiqueta $(\mathbf{m})$; observar que no es posible que $z_{h}<z_{j}$, pues $z_{j}<z_{r}$ y $z_{h} \| z_{r}$. Luego, necesariamente $z_{j}<z_{h}$. Como $z_{h} \| z_{r}$, existe un elemento maximal $z_{i}$ con $i<r$ tal que $z_{h}<z_{i}$ en $\mathbf{P}$. Luego $z_{j}<z_{i}$ en $\mathbf{P}$, lo cual es una contradicción por el item $(v)$ del Lema 6.2.4.

Por otro lado, si $z_{j}$ tiene etiqueta (d) entonces $z_{j}<z_{h}$. Como $z_{h} \| z_{r}$ existe un elemento maximal $z_{i}$ con $i<r$ tal que $z_{h}<z_{i}$ en $\mathbf{P}$. Luego $z_{j}<z_{i}$, lo que es absurdo por el item (iv) del Lema 6.2.4. Concluimos que $z_{h} \| z_{j}$ cuando $z_{h}$ tiene etiqueta (m). $\mathrm{Si} z_{h}$ es minimal entonces, observar que $z_{j}$ no puede tener etiqueta (u) ya que $j \geq r$ y $z_{h} \| z_{r}$. Luego, si la etiqueta de $z_{j}$ es $(\mathbf{m})$ entonces $z_{h}<z_{j}$ en $\mathbf{P}$. El hecho de que $z_{j}<z_{r}$ implica que $z_{h}<z_{r}$, lo cual es una contradicción. Por lo tanto, $z_{h} \| z_{j}$ cuando $z_{h}$ es minimal.

En el siguiente Teorema veremos que la condición del Lema 4.1.2 es suficiente para los posets $k$-tree que son $C P T$.

Teorema 6.2.8. Sea $\mathbf{P}=(X, P)$ un poset $k$-tree. Entonces $\mathbf{P}$ admite un modelo $C P T$ si y solo si $D[z]$ es $C I$ para todo z maximal de $\mathbf{P}$.

\section{Demostración:}

La implicación directa vale en general como lo demuestra el Lema 4.1.2.

Para demostrar la proposición recíproca, primero asumimos que $\mathbf{P}$ no tiene falsos gemelos. Probaremos que si tenemos un poset $\mathbf{P}=(X, P) k$-tree sin falsos gemelos tal que $\mathbf{P}(D[z])$ es $C I$ para todo $z$ elemento maximal de $\mathbf{P}$, entonces $\mathbf{P}$ admite un modelo $C P T$ en el cual ningún camino se reduce a un vértice y ningún par de caminos tiene un extremo coincidente. Procederemos por inducción sobre $|X|$. 
Si $|X|=k$ o $k+1$ entonces $\mathbf{P}$ es una cadena y la demostración es inmediata.

Si no, por el Lema 6.2.5 existe un buen orden constructivo de $\mathbf{P} z_{1}, z_{2}, \ldots z_{r}, z_{r+1}, \ldots, z_{n}$ donde $z_{r}$ es el último maximal que fue agregado en el proceso constructivo y $z_{i} \in D\left[z_{r}\right]$ si $i \geq r+1$.

Sea $X^{\prime}=\left\{z_{1}, z_{2}, \ldots, z_{r-1}\right\}$. Entonces existe un modelo $C P T R_{\mathbf{P}^{\prime}}=\left(W_{z_{i}}^{\prime}\right)_{z_{i} \in X^{\prime}}$ sobre un árbol huésped $T^{\prime}$, en el cual ningún camino se reduce a un punto y ningún par de caminos tiene un extremo coincidente.

Por otro lado sea $X^{\prime \prime}=D_{\mathbf{P}}\left[z_{r}\right]$. Por hipótesis $\mathbf{P}^{\prime \prime}=\mathbf{P}\left(X^{\prime \prime}\right)$ es $C I$. Luego es posible obtener un modelo $C I R_{\mathbf{P}^{\prime \prime}}=\left(W_{z_{i}}^{\prime \prime}\right)_{z_{i} \in D\left[z_{r}\right]}$, donde ningún camino se reduce a un vértice y ningún par de caminos tiene un extremo coincidente [6] .

En lo que sigue, reacomodaremos los modelos $R_{\mathbf{P}^{\prime}}$ y $R_{\mathbf{P}^{\prime \prime}}$ de manera tal que su superposición nos permita obtener un modelo $C P T$ de $\mathbf{P}$. Por el primer ítem del Lema 6.2.7 se tiene que $X^{\prime} \cap X^{\prime \prime}=\left\{z_{j_{1}}, z_{j_{2}}, \ldots, z_{j_{k}}\right\}$ con $z_{j_{1}}<z_{j_{2}}<\ldots<z_{j_{k}}$ en $\mathbf{P}$; luego los vértices $z_{j_{i}}$ están representados tanto en el modelo $R_{\mathbf{P}^{\prime}}$ como en $R_{\mathbf{P}^{\prime \prime}}$.

En ambos modelos los caminos no tienen extremos coincidentes; luego es claro que, subdividiendo aristas donde sea necesario, en el modelo $R_{\mathbf{P}^{\prime}}$ o en el modelo de $R_{\mathbf{P}^{\prime \prime}}$, se puede asumir que las longitudes de $W_{z_{j_{i}}}^{\prime}$ y $W_{z_{j_{i}}}^{\prime \prime}$ son iguales y que la posición relativa de $W_{z_{j_{i}}}^{\prime}$ en $W_{z_{j_{k}}}^{\prime}$ es igual a la posición relativa de $W_{z_{j_{i}}}^{\prime \prime}$ en $W_{z_{j_{k}}}^{\prime \prime}$.

Sea $R$ el modelo por contención de caminos que se obtiene uniendo ambos modelos de forma que, para cada $1 \leq i \leq k, W_{z_{j_{i}}}^{\prime}$ se identifica con $W_{z_{j_{i}}}^{\prime \prime}$. Y a su vez, la parte de $W_{z_{r}}^{\prime \prime}$ que no está cubierta por $W_{z_{j_{k}}}^{\prime \prime}$ se representa en $T^{\prime}$ agregando 2 nuevas ramas en cada uno de los extremos (ver Figura 6.3).

A continuación probaremos que $R$ es un modelo $C P T$ de $\mathbf{P}$. En este modelo llamamos $W_{z}$ al camino correspondiente al elemento $z$, siendo $W_{z_{i}}=W_{z_{i}}^{\prime}$ si $i<r$ y $W_{z_{i}}=W_{z_{i}}^{\prime \prime}$ si $i \geq r$. Primero veamos que si $z_{i}<z_{j}$ en $\mathbf{P}$ entonces $W_{z_{i}} \subset W_{z_{j}}$.

Si $z_{i}$ y $z_{j}$ pertenecen ambos a $X^{\prime}$ o $X^{\prime \prime}$ entonces $z_{i}<z_{j}$ en $\mathbf{P}^{\prime}$ o $\mathbf{P}^{\prime \prime}$, respectivamente, lo cual implica que $W_{z_{i}}^{\prime} \subset W_{z_{j}}^{\prime}$ o $W_{z_{i}}^{\prime \prime} \subset W_{z_{j}}^{\prime \prime}$. Por lo tanto $W_{z_{i}} \subset W_{z_{j}}$. 
Si $z_{i} \in X^{\prime}$ y $z_{j} \in X^{\prime \prime}$, por el Lema 6.2 .7 se tiene que $z_{i}=z_{j_{l_{i}}}$ con $1 \leq l_{i} \leq k$, es decir $z_{i} \in X^{\prime} \cap X^{\prime \prime}$. Luego $z_{i}=z_{j_{l_{i}}}<z_{j}$ en $\mathbf{P}^{\prime \prime}$, y como vimos previamente, se tiene que $W_{z_{j_{i}}}=W_{z_{i}} \subset W_{z_{j}}$. El caso $z_{i} \in X^{\prime \prime} \mathrm{y} z_{j} \in X^{\prime}$ se analiza análogamente.

Nos queda probar que si $W_{z_{i}}$ y $W_{z_{j}}$ son caminos pertenecientes a $R$ tales que $W_{z_{i}} \subset$ $W_{z_{j}}$ entonces $z_{i}<z_{j}$ en $\mathbf{P}$. Es claro que si ambos caminos pertenecen a $R_{\mathbf{P}^{\prime}}$ entonces $z_{i}<z_{j}$ en $\mathbf{P}^{\prime}$ lo que implica que $z_{i}<z_{j}$ en $\mathbf{P}$. Análogamente se prueba que si ambos caminos pertenecen a $R_{\mathbf{P}^{\prime \prime}}$ entonces $z_{i}<z_{j}$ en $\mathbf{P}$.

Si $W_{z_{i}} \in R_{\mathbf{P}^{\prime}}, W_{z_{j}} \in R_{\mathbf{P}^{\prime \prime}}$ y $W_{z_{i}} \subset W_{z_{j}}$, como también sabemos que $W_{z_{j}} \subset W_{z_{r}}$, se concluye que $W_{z_{i}} \subset W_{z_{r}}$. Esto a su vez implica que $W_{z_{i}} \subseteq W_{z_{j_{k}}}$. Estos dos últimos conjuntos pertenecen a $R_{\mathbf{P}^{\prime}}$, entonces la inclusión implica $z_{i} \leq z_{j_{k}}$. Además $W_{z_{j_{k}}}$ y $W_{r}$ están en $R_{\mathbf{P}^{\prime \prime}}$, por lo que $W_{z_{j_{k}}} \subset W_{z_{r}}$, entonces $z_{j_{k}}<z_{r}$. Por transitividad $z_{i}<z_{r}$. Por lo tanto $z_{i} \in X^{\prime \prime}$ y $W_{z_{i}} \in R_{\mathbf{P}^{\prime \prime}}$, además sabemos que $W_{z_{j}} \subset R_{\mathbf{P}^{\prime \prime}}$. Por ende $W_{z_{i}} \subset W_{z_{j}}$ implica que $z_{i}<z_{j}$.

Si $W_{z_{i}} \in R_{\mathbf{P}^{\prime \prime}}$ y $W_{z_{j}} \in R_{\mathbf{P}^{\prime}}$, consideramos dos casos: $z_{j} \in X^{\prime} \cap X^{\prime \prime}$ o no.

Si $z_{j} \in X^{\prime} \cap X^{\prime \prime}$, entonces $W_{z_{j}} \in R_{\mathbf{P}^{\prime \prime}}$. Así, se tiene que $z_{i}<z_{j}$ en $\mathbf{P}^{\prime \prime}$.

Consideremos el caso $z_{j} \notin X^{\prime} \cap X^{\prime \prime}$, si $z_{i} \in X^{\prime}$, entonces $W_{z_{i}}$ también está en $R_{\mathbf{P}^{\prime}}$. Entonces $W_{z_{i}} \subset W_{z_{j}}$ en $R_{\mathbf{P}^{\prime}}$ implica $z_{i}<z_{j}$. Si $z_{i} \notin X^{\prime}$ entonces $z_{i}=z_{r}$ o $i>r$. Si $z_{i}=z_{r}$, es absurdo porque todos los vértices de $W_{z_{r}}$ no están en el árbol huésped de $R_{\mathbf{P}^{\prime}}$ (lo más que llegan a compartir es $W_{z_{j_{2}}}$ ).

Si $z_{i} \neq z_{r}$, con $i>r, W_{z_{i}} \subseteq W_{z_{j}}$ implica $W_{z_{i}} \subseteq W_{z_{j_{k}}}$. Entonces $W_{z_{i}} \subset W_{z_{j_{k}}} \subset W_{z_{r}}$ en $R_{\mathbf{P}^{\prime \prime}}$ implica $z_{i}<z_{j_{k}}<z_{r}$. Entonces $z_{i}$ tiene etiqueta $(\mathbf{d})$ o $(\mathbf{m})$.

Sin perdida de generalidad, asumimos que $z_{i}$ tiene etiqueta (d), cuando él fue agregado miraba a $z_{j_{k}}$ y $z_{r}$ y, por etiqueta (d), $U\left(z_{j_{k}}\right)=z_{r}$ (en ese estadio de la construcción). Entonces, en ese momento $z_{j_{k}}$ se relacionaba con un único maximal que es $z_{r}$. Si $z_{r}$ es el único maximal, entonces $X^{\prime \prime}=X$, y $W_{z_{i}}, W_{z_{j}} \in R_{\mathbf{P}^{\prime \prime}}$. Si $z_{r}$ no fuera el único maximal, por construcción, al momento de ser agregado, $z_{j_{1}}$ y $z_{j_{k}}$ estaba en una cadena junto a otro maximal $z_{k}$. Eso es absurdo porque contradice la etiqueta (d) de 
$z_{i}$.

Concluimos que $R$ es un modelo $C P T$ del poset $\mathbf{P}$ en el cual todos los caminos tiene longitud mayor o igual a uno.

Podría ocurrir que existan caminos que tengan un extremo coincidente en $R$. Como asumimos que $\mathbf{P}$ no tiene falsos gemelos, por el Lema 4.1.13 podemos asegurar que existe un modelo $C P T$ de $\mathbf{P}$ en el cual ningún par de caminos tiene un extremo coincidente.

Si $\mathbf{P}$ tiene falsos gemelos, por cada clase de equivalencia de falsos gemelos podemos dejar un representante. Así, por lo demostrado previamente, tenemos un nuevo poset $C P T \mathbf{P}_{\mathbf{1}}$, el cual es $k$-tree y no tiene falsos gemelos. Luego, por la Observación 4.1.6, $\mathbf{P}$ es $C P T$.

El Teorema 6.2.8 muestra que en la clase de los posets $k$-tree, con $k \geq 2$, la condición necesaria para ser CPT dada por el Lema 4.1.2 es también suficiente. Luego el Teorema 4.1.5 y el siguiente lema completan la demostración del Teorema 6.2.10 que brinda una caracterización por subposets prohibidos de los posets $k$-tree.

Lema 6.2.9. Sea $\mathbf{P}=(X, P)$ un poset, $\mathbf{H}$ un subposet de $\mathbf{P}$ 3-irreducible, con $h(\mathbf{H})<$ $h(\mathbf{P}) ; y \mathbf{B}, \mathbf{C}, \mathbf{E}_{\mathbf{n}}$ y $\mathbf{F}_{\mathbf{n}}$, con $\mathbf{n} \geq 0$, los posets descriptos en las Figuras 2.5 y 2.6.

i) Si $\mathbf{P}$ es 2-tree entonces $\boldsymbol{H}$ debe ser $\mathbf{B}$ o $\mathbf{B}^{d}$.

ii) Si $\mathbf{P}$ es 3-tree entonces $\mathbf{H}$ debe ser $\mathbf{B}, \mathbf{B}^{d}, \mathbf{C}, \mathbf{C}^{d}, \mathbf{E}_{\mathbf{n}}$ o $\mathbf{E}_{\mathbf{n}}^{d}$ con $\mathbf{n} \geq 0$.

iii) Si $\mathbf{P}$ es $k$-tree con $k \geq 4$ entonces $\mathbf{H}$ debe ser $\mathbf{B}, \mathbf{B}^{d}, \mathbf{C}, \mathbf{C}^{d}, \mathbf{E}_{\mathbf{n}}, \mathbf{E}_{\mathbf{n}}^{d}$ o $\mathbf{F}_{\mathbf{n}}$ con $\mathbf{n} \geq 0$.

Demostración: Los posets 3-irreducibles son los posets descriptos en las Figuras 2.5 y 2.6 y sus respectivos duales. Sea $\mathbf{H}$ un poset 3-irreducible, subposet de un poset 
$k$-tree.

Como los grafos $k$-tree son cordales, es decir, no tienen ciclos sin cuerdas inducidos, los posets $k$-tree son acíclicos. Resulta así que $\mathbf{H}$ no es ninguno de los posets: D, $\mathbf{C X}_{1}, \mathbf{C X}_{2}, \mathbf{C X}_{3}, \mathbf{E X}_{1}, \mathbf{E X}_{2}, \mathbf{F X}_{1}, \mathbf{F X}_{2}$ ni sus poset duales; $\mathbf{A}_{\mathbf{n}} \mathbf{I}_{\mathbf{n}}, \mathbf{G}_{\mathbf{n}}, \mathbf{J}_{\mathbf{n}}, \mathbf{H}_{\mathbf{n}}$, con $\mathbf{n} \geq 0$, ni sus respectivos duales.

Para demostrar $(i)$ asumimos que $\mathbf{P}$ es 2-tree. Luego $h(\mathbf{H})<3$ por lo que $\mathbf{H}$ debe ser alguno de los posets $\mathbf{B}$ o $\mathbf{B}^{d}$.

Si $\mathbf{P}$ es 3-tree entonces $h(\mathbf{H})<4$. Luego $\mathbf{H}$ debe ser algunos de los posets $\mathbf{B}, \mathbf{B}^{d}, \mathbf{C}$, $\mathbf{C}^{d}, \mathbf{E}_{\mathbf{n}}$ o $\mathbf{E}_{\mathbf{n}}^{d}$, con $\mathbf{n} \geq 0$. Así hemos demostrado $\left.i i\right)$.

Si $k \geq 4$ entonces $h(\mathbf{H})<k+1$. Luego $\mathbf{H}$ debe ser alguno de los posets $\mathbf{B}, \mathbf{B}^{d}, \mathbf{C}$, $\mathbf{C}^{d}, \mathbf{E}_{\mathbf{n}}, \mathbf{E}_{\mathbf{n}}^{d}$ o $\mathbf{F}_{\mathbf{n}}$, con $\mathbf{n} \geq 0$. Observar que $\mathbf{F}_{\mathbf{n}}=\mathbf{F}_{\mathbf{n}}^{d}$. Así queda probado iii).

Teorema 6.2.10. Sea $\mathbf{P}=(X, P)$ un poset, las siguientes proposiciones son ciertas.

i) Sea $\mathbf{P}$ es 2-tree. Entonces $\mathbf{P}$ es CPT si y solo si $\mathbf{P}$ no tiene como subposets $\widehat{\mathbf{B}}$ ni $\widehat{\boldsymbol{B}^{d}}$.

ii) Sea $\mathbf{P}$ es 3-tree. Entonces $\mathbf{P}$ es CPT si y solo si $\mathbf{P}$ no tiene como subposets $\widehat{\mathbf{B}}$, $\widehat{\mathbf{B}^{d}}, \widehat{\mathbf{C}}, \widehat{\mathbf{C}^{d}}, \widehat{\mathbf{E}_{\mathbf{n}}} n i \widehat{\mathbf{E}_{\mathbf{n}}^{d}}$ con $\mathbf{n} \geq 0$.

iii) Sea $\mathbf{P}$ es $k$-tree con $k \geq 4$. Entonces $\mathbf{P}$ es CPT si y solo si $\mathbf{P}$ no tiene como subposets $\widehat{\mathbf{B}}, \widehat{\boldsymbol{B}^{d}}, \widehat{\mathbf{C}}, \widehat{\mathbf{C}^{d}}, \widehat{\mathbf{E}_{\mathbf{n}}}, \widehat{\mathbf{E}_{\mathbf{n}}^{d}}$ ni $\widehat{\mathbf{F}_{\mathbf{n}}} \operatorname{con} \mathbf{n} \geq 0$.

Los posets $\widehat{\mathbf{B}}, \widehat{\mathbf{B}^{d}}, \widehat{\mathbf{C}}, \widehat{\mathbf{C}^{d}}, \widehat{\mathbf{E}_{\mathbf{n}}}, \widehat{\mathbf{E}_{\mathbf{n}}^{d}} y \widehat{\mathbf{F}_{\mathbf{n}}}$, con $\mathbf{n} \geq 0$, están en la Figura 6.4 .

Lema 6.2.11. Sean $\mathbf{P}=(X, P)$ y $\mathbf{P}^{\prime}=\left(X, P^{\prime}\right)$ posets asociados. Si $\widehat{\mathbf{B}}$ o cualquiera de los posets $\widehat{\mathbf{C}}, \widehat{\mathbf{E}_{\mathbf{n}}}$ o $\widehat{\mathbf{F}_{\mathbf{n}}}$, descriptos en la Figura 6.4 , es un subposet de $\mathbf{P}$, entonces o bien $\widehat{\mathbf{B}}, \widehat{\mathbf{B}^{d}}, \widehat{\mathbf{C}}, \widehat{\mathbf{C}^{d}}, \widehat{\mathbf{E}_{\mathbf{n}}}, \widehat{\mathbf{E}_{\mathbf{n}}^{d}}$ o $\widehat{\mathbf{F}_{\mathbf{n}}}$ es subposet de $\mathbf{P}^{\prime}$, respectivamente; o bien $\widehat{\mathbf{B}}^{d}$, $\widehat{\mathbf{B}}^{d}, \widehat{\mathbf{C}}^{d}, \widehat{\mathbf{C}}^{d}, \widehat{\mathbf{E}}_{\mathbf{n}}^{d}, \widehat{\mathbf{E}}_{\mathbf{n}}^{d}$ o $\widehat{\mathbf{F}}_{\mathbf{n}}^{d}$ es subposet de $\mathbf{P}^{\prime}$, respectivamente. 

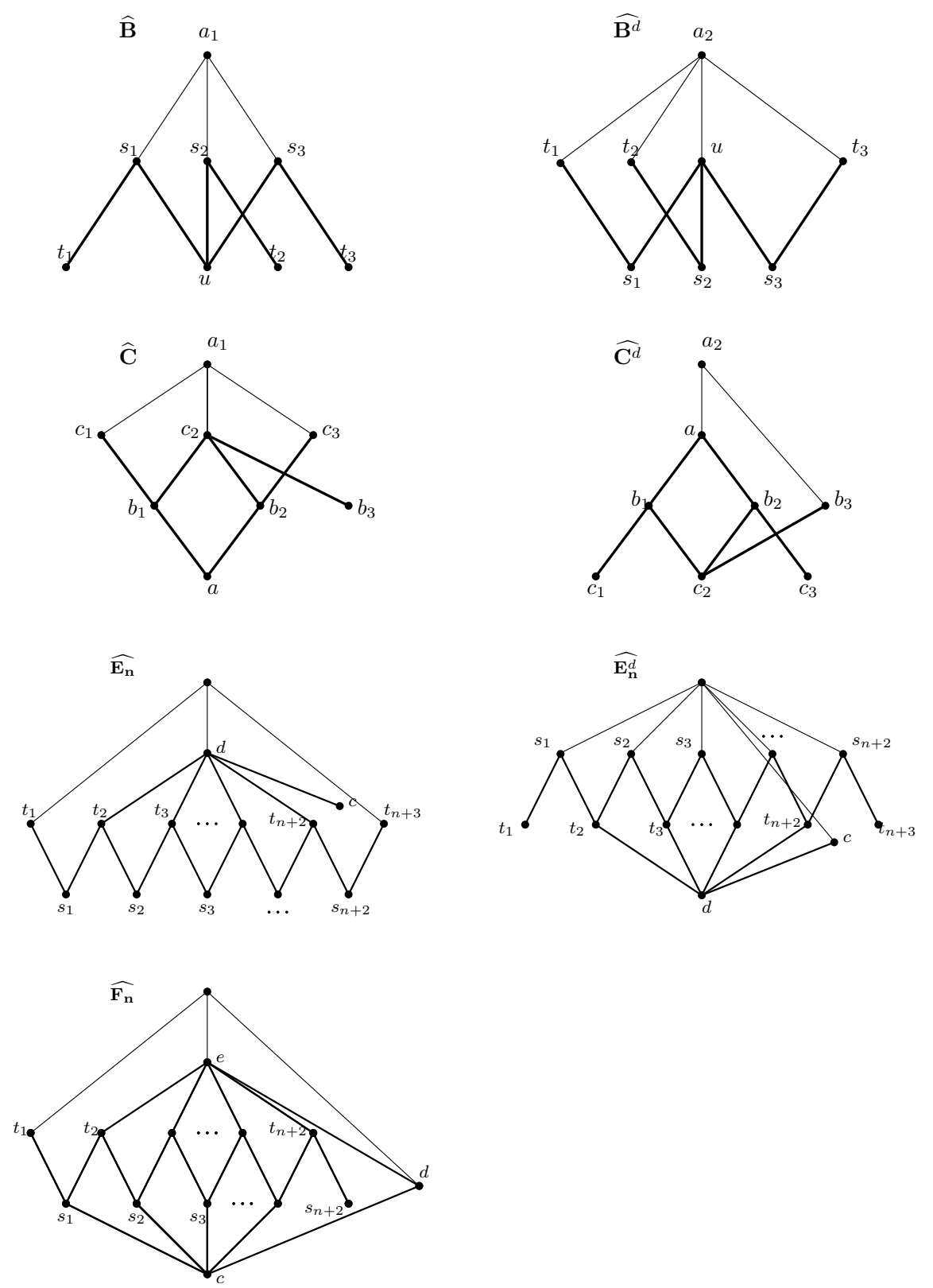

Figura 6.4: Los posets $\widehat{\mathbf{B}}$ y $\widehat{\mathbf{B}^{d}}$ son 2-tree pero no $C P T$. $\widehat{\mathbf{C}}, \widehat{\mathbf{C}^{d}}, \widehat{\mathbf{E}_{\mathbf{n}}}$ y $\widehat{\mathbf{E}_{\mathbf{n}}^{d}}$ para $\mathbf{n} \geq 0$ no son posets 3 -tree. Sin embargo, pueden ser subposet de un poset 3-tree; además ninguno es $C P T$. $\widehat{\mathbf{F}_{\mathbf{n}}}$, con $\mathbf{n} \geq 0$, puede ser subposets de un poset 4 -tree pero no es CPT. 

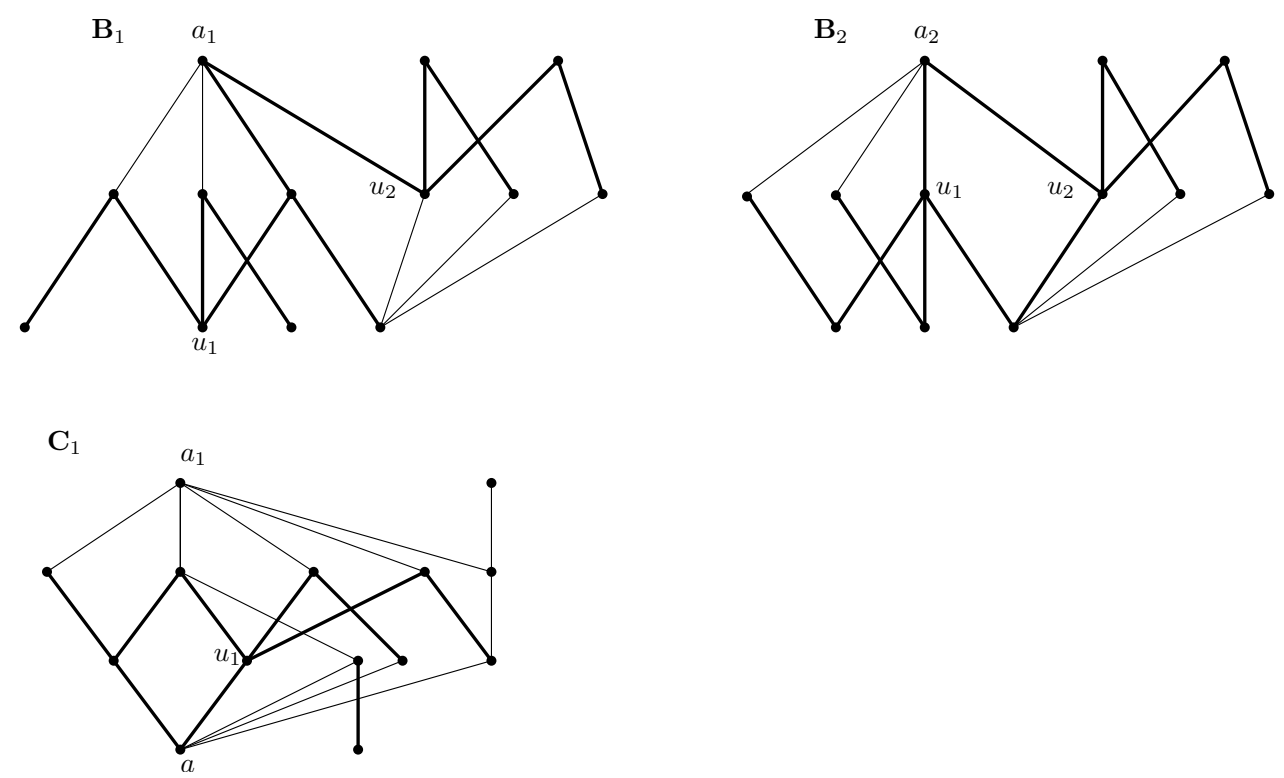

Figura 6.5: Los posets $\mathbf{B}_{1}$ y $\mathbf{B}_{2}$ son 2-tree, el poset $\mathbf{C}_{1}$ es 3-tree. Observar que $\mathbf{B}_{2}$ tiene a $\widehat{\mathbf{B}}$ como subposet, $\mathbf{B}_{1}$ tiene a $\widehat{\mathbf{B}^{d}}$ como subposet y $\mathbf{C}_{1}$ tiene a $\widehat{\mathbf{C}}$ como subposet. Luego, por el Teorema 6.2.10, no son CPT.

Demostración: Sea $\mathbf{P}$ un poset que contiene a $\widehat{\mathbf{B}}$ como subposet. Los módulos no triviales de $\widehat{\mathbf{B}}$ son: su vértice universal, que llamaremos $y$, y los vértices del subposet B, descripto en la Figura 2.5. Al conjunto de vértices de $\mathbf{B}$ lo denotaremos por $X$. Como $\mathbf{P}$ y $\mathbf{P}^{\prime}$ son asociados (tienen el mismo grafo de comparalibidad) podemos determinar el vértice $y^{\prime}$ y el conjunto $X^{\prime}$ de vértices de $\mathbf{P}^{\prime}$ correspondientes al vértice $y$ y al conjunto $X$, respectivamente.

Por el segundo ítem del Corolario 2.3.9 tenemos que el subposet de $\mathbf{P}^{\prime}$ inducido por los vértices de $X^{\prime}$ es $\mathbf{B}$ o $\mathbf{B}^{d}$.

Observar que en $\mathbf{P}^{\prime}$ el vértice $y^{\prime}$ debe ser mayor que todos los vértices de $X^{\prime}$ o debe ser menor que todos ellos, luego en $\mathbf{P}^{\prime}$ tenemos como subposet a $\widehat{\mathbf{B}}, \widehat{\mathbf{B}}^{d}, \widehat{\mathbf{B}}^{d}$ o bien $\widehat{\mathbf{B}}^{d}$.

Para los posets $\widehat{\mathbf{C}}, \widehat{\mathbf{E}_{\mathbf{n}}}$ y $\widehat{\mathbf{F}_{\mathbf{n}}}$ la demostración es análoga. 
Como consecuencia del lema anterior y del Teorema 6.2.10, probamos que la clase de posets $k$-tree dualmente- $C P T$ coincide con la clase $k$-tree fuertemente- $C P T$ y obtenemos una caracterización de la clase de posets $k$-tree fuertemente- $C P T$, para $k \geq 2$.

Teorema 6.2.12. Sea $\mathbf{P}=(X, P)$ un poset $k$-tree con $k \geq 2$. Las siguientes condiciones son equivalentes.

i) $\mathbf{P}$ es fuertemente-CPT.

ii) $\mathbf{P}$ es dualmente-CPT.

iii) $\mathbf{P}$ no contiene $a \widehat{\mathbf{B}}, \widehat{\mathbf{B}^{d}}, \widehat{\mathbf{C}}, \widehat{\mathbf{C}^{d}}, \widehat{\mathbf{E}_{\mathbf{n}}}, \widehat{\mathbf{E}_{\mathbf{n}}^{d}}, \widehat{\mathbf{F}_{\mathbf{n}}}$, con $\mathbf{n} \geq 0$, ni a sus duales como subposet, ver la Figura 6.4.

Observar que para $k=2,3$ la familia de prohibidos no es minimal. Para los posets 2-tree que son fuertemente-CPT, los posets $\widehat{\mathbf{B}}, \widehat{\mathbf{B}^{d}}$ y sus duales constituyen la familia de prohibidos minimal. Mientras que la familia de prohibidos minimal de los 3-tree fuertemente-CPT está formada por $\widehat{\mathbf{B}}, \widehat{\mathbf{B}^{d}}, \widehat{\mathbf{C}}, \widehat{C^{d}}, \widehat{\mathbf{E}_{\mathbf{n}}}, \widehat{\mathbf{E}_{\mathbf{n}}^{d}}$, con $\mathbf{n} \geq 0$, y sus respectivos duales.

Demostración: La implicación $i$ ) entonces $i$ ) es cierta aún cuando $\mathbf{P}$ no sea $k$-tree. Si $\mathbf{P}$ es dualmente-CPT entonces, por definición de dualmente-CPT y el Teorema 6.2.10, los posets $\widehat{\mathbf{B}}, \widehat{\mathbf{B}^{d}}, \widehat{\mathbf{C}}, \widehat{\mathbf{C}^{d}} \widehat{\mathbf{E}_{n}}, \widehat{\mathbf{E}_{n}^{d}}, \widehat{\mathbf{F}_{n}}$, con $\mathbf{n} \geq 0$, y sus respectivos duales no pueden ser subposets de $\mathbf{P}$. Así hemos probado la implicación ii) entonces iii).

Queda demostrar la implicación iii) entonces $i$ ). Por iii) del Teorema 6.2.10, $\mathbf{P}$ es $C P T$. Asumimos, con el fin de obtener una contradicción, que $\mathbf{P}$ no es fuertemente$C P T$. Sea $\mathbf{P}^{\prime}$ un poset no $C P T$ asociado a $\mathbf{P}$. Por el Teorema 6.2.10 $\mathbf{P}^{\prime}$ contiene como subposet, a alguno de los posets $\widehat{\mathbf{B}}, \widehat{\mathbf{B}^{d}}, \widehat{\mathbf{C}}, \widehat{\mathbf{C}^{d}}, \widehat{\mathbf{E}_{\mathbf{n}}}, \widehat{\mathbf{E}_{\mathbf{n}}^{d}}, \widehat{\mathbf{F}_{\mathbf{n}}}$ con $\mathbf{n} \geq 0$. Luego, por el Lema 6.2.11, $\mathbf{P}$ contiene como subposet a alguno de los siguiente posets: $\widehat{\mathbf{B}}, \widehat{\mathbf{B}^{d}}, \widehat{\mathbf{C}}$, 

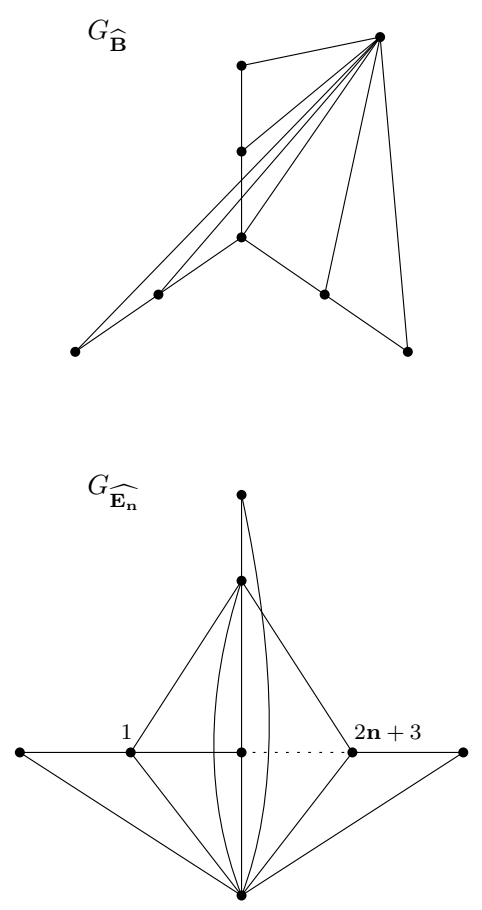
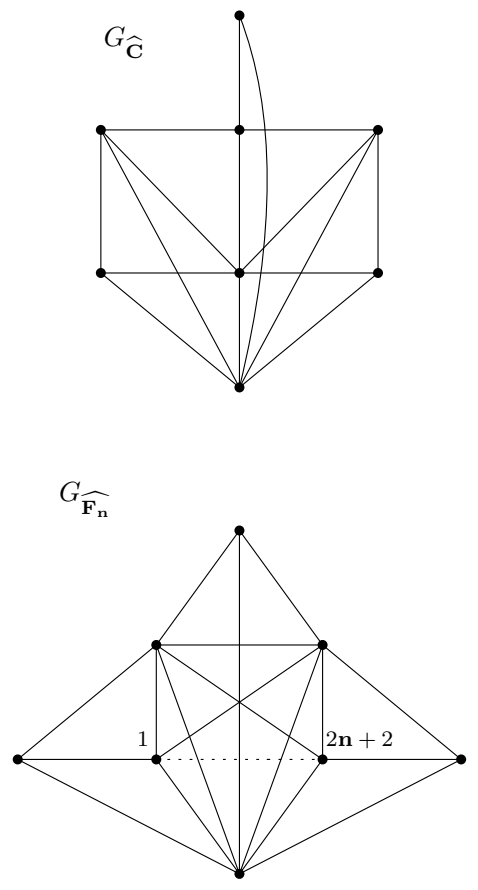

Figura 6.6: Los grafos de comparabilidad de los posets $\widehat{\mathbf{B}}, \widehat{\mathbf{C}}, \widehat{\mathbf{E}_{\mathbf{n}}}, \widehat{\mathbf{F}_{\mathbf{n}}}$ en la Figura 6.4. $\widehat{\mathbf{C}^{d}}, \widehat{\mathbf{E}_{\mathbf{n}}}, \widehat{\mathbf{E}_{\mathbf{n}}^{d}}$ o $\widehat{\mathbf{F}}_{\mathbf{n}}, \widehat{\mathbf{B}}^{d}, \widehat{\mathbf{B}}^{d}, \widehat{\mathbf{C}}^{d}, \widehat{\mathbf{C}}^{d}, \widehat{\mathbf{E}}_{\mathbf{n}}^{d}, \widehat{\mathbf{E}}_{\mathbf{n}}^{d}, \widehat{\mathbf{F}}_{\mathbf{n}}^{d}$. Esto contradice nuestra asunción sobre $\mathbf{P}$.

El siguiente resultado será utilizado en la próxima sección en la caracterización de grafos 2-tree que son $C P T$.

Lema 6.2.13. Si $\mathbf{P}^{\prime}$ es un poset asociado a $\mathbf{B}_{1}$ o $\mathbf{B}_{2}$ en la Figura 6.5 entonces tiene a $\widehat{\mathbf{B}^{d}}$ como subposet.

Demostración: Observemos que el grafo de comparabilidad de $\mathbf{P}^{\prime}$ tiene una única clase de implicancia. Luego, por la Proposición 2.3.2, $\mathbf{P}^{\prime}$ tiene a su dual como un único poset asociado. Por lo tanto, podemos asumir $\mathbf{P}^{\prime}=\mathbf{B}_{1}{ }^{d}\left({ }_{0} \mathbf{B}_{2}{ }^{d}\right)$. Es fácil ver en la Figura 6.5 que $\mathbf{B}_{1}\left(\right.$ o $\left.\mathbf{B}_{2}\right)$ tiene al dual de $\widehat{\mathbf{B}^{d}}$ como subposet. Luego, $\mathbf{P}^{\prime}$ tiene a $\widehat{\mathbf{B}^{d}}$ como subposet. 


\subsection{Grafos CPT $k$-trees}

Recordemos que los grafos fuertemente- $C P T$ y grafos dualmente- $C P T$ son los grafos de comparabilidad de los posets fuertemente- $C P T$ y posets dualmente- $C P T$, respectivamente.

Como corolario del Teorema 4.2.3 y el hecho que todo árbol es un grafo de comparabilidad tenemos el siguiente resultado.

Teorema 6.3.1. Todo árbol es un grafo fuertemente-CPT.

En el siguiente Teorema presentamos una caracterización de los grafos $k$-tree que son dualmete- $C P T$ y probamos que son exactamente los grafos $k$-tree que son fuertementeCPT.

Teorema 6.3.2. Sea $G$ un grafo de comparabilidad y $k$-tree. Las siguientes condiciones son equivalentes.

i) $G$ es fuertemente-CPT.

ii) $G$ es dualmente-CPT.

iii) $G$ no contiene a ninguno de los grafos $G_{\widehat{\mathbf{B}}}, G_{\widehat{\mathbf{C}}}, G_{\widehat{\mathbf{E}_{n}}}$ ni $G_{\widehat{\mathbf{F}_{\mathbf{n}}}}$ con $\mathbf{n} \geq 0$ de la Figura 6.6 como subgrafo inducido.

Demostración: Por el Teorema 6.2.12 es cierta la proposición $i$ ) si y solo si ii). Para probar $i$ i) implica $i i i)$, sea $G$ dualmente- $C P T$. Entonces existe un poset $\mathbf{P}$ dualmente$C P T$ tal que $G=G_{\mathbf{P}}$. Asumamos, para llegar a una contradicción, que $G$ tiene como subgrafo inducido a alguno de los siguientes grafos: $G_{\widehat{\mathbf{B}}}, G_{\widehat{\mathbf{C}}}, G_{\widehat{\mathbf{E}_{\mathbf{n}}}}$ o $G_{\widehat{\mathbf{F}_{\mathbf{n}}}}$. Entonces 
$\mathbf{P}$ contiene un subposet $\mathbf{P}^{\prime}$ asociado a $\widehat{\mathbf{B}}, \widehat{\mathbf{C}}, \widehat{\mathbf{E}_{\mathbf{n}}}$ ○ $\widehat{\mathbf{F}_{\mathbf{n}}}$. Luego, por el Lema 6.2 .11 , se tiene los siguiente casos:

- Si $\mathbf{P}^{\prime}$ es asociado a $\widehat{\mathbf{B}}$ entonces $\mathbf{P}^{\prime}=\widehat{\mathbf{B}}, \widehat{\mathbf{B}}^{d}, \widehat{\mathbf{B}}^{d}$ o $\widehat{\mathbf{B}}^{d}$.

- Si $\mathbf{P}^{\prime}$ es asociado a $\widehat{\mathbf{C}}$ entonces $\mathbf{P}^{\prime}=\widehat{\mathbf{C}}, \widehat{\mathbf{C}}^{d}, \widehat{\mathbf{C}}^{d}{ }_{\mathrm{o}} \widehat{\mathbf{C}}^{d}$.

- Si $\mathbf{P}^{\prime}$ es asociado a $\widehat{\mathbf{E}_{\mathbf{n}}}$ entonces $\mathbf{P}^{\prime}=\widehat{\mathbf{E}_{\mathbf{n}}}, \widehat{\mathbf{E}}_{\mathbf{n}}^{d}, \widehat{\mathbf{E}}_{\mathbf{n}}^{d}$ o $\widehat{\mathbf{E}}_{\mathbf{n}}^{d}$.

- Si $\mathbf{P}^{\prime}$ es asociado a $\widehat{\mathbf{F}_{\mathbf{n}}}$ entonces $\mathbf{P}^{\prime}=\widehat{\mathbf{F}_{\mathbf{n}}}$ o $\widehat{\mathbf{F}}_{\mathbf{n}}^{d}$.

En cualquiera de los casos anteriores, por el Teorema 6.2.12, el poset $\mathbf{P}$ no es dualmente$C P T$, lo cual es una contradicción. Así queda demostrado ii) implica iii). Para demostrar iii) implica $i$ ), con el objetivo de llegar a una contradicción, asumimos que $G$ no es fuertemente- $C P T$. Entonces existe un poset $\mathbf{P}$, que no es $C P T$, tal que $G=G_{\mathbf{P}}$. Por el Teorema 6.2.10, $\mathbf{P}$ tiene como subposet a alguno de los siguientes posets: $\widehat{\mathbf{B}}, \widehat{\mathbf{B}^{d}}, \widehat{\mathbf{C}}, \widehat{\mathbf{C}^{d}}, \widehat{\mathbf{E}_{\mathbf{n}}}, \widehat{\mathbf{E}_{\mathbf{n}}^{d}}$ o $\widehat{\mathbf{F}_{\mathbf{n}}}$. Luego, en cualquiera de los casos $G_{\mathbf{P}}$, tiene como subgrafo inducido a alguno de los grafos $G_{\widehat{\mathrm{B}}}, G_{\widehat{\mathrm{C}}}, G_{\widehat{\mathbf{E}_{\mathbf{n}}}}$ o $G_{\widehat{\mathbf{F}_{\mathbf{n}}}}$ con $\mathbf{n} \geq 0$, lo cual contradice iii).

Los siguientes corolarios son consecuencia de los teoremas 6.2.12 y 6.3.2.

Corolario 6.3.3. Sea $G$ un grafo de comparabilidad y 2-tree. Las siguientes condiciones son equivalentes.

i) G es fuertemente-CPT.

ii) $G$ es dualmente-CPT.

iii) $G$ no contiene al grafo $G_{\widehat{\mathrm{B}}}$ en la Figura 6.6 como subgrafo inducido.

Corolario 6.3.4. Sea $G$ un grafo de comparabilidad y 3-tree. Las siguientes condiciones son equivalentes. 
i) $G$ es fuertemente-CPT.

ii) $G$ es dualmente-CPT.

iii) $G$ no contiene $a G_{\widehat{\mathrm{B}}}$, ni a $G_{\widehat{\mathrm{C}}}$, ni a $G_{\widehat{\mathbf{E}}_{\mathbf{n}}}$ con $\mathbf{n} \geq 0$, en la Figura 6.6, como subgrafos inducidos.

Notar que, de forma similar a lo que ocurre con los posets split que son $C P T$, aunque el Teorema 6.2.10 nos brinda una caracterización por subposets prohibidos de los posets $k$-tree, con $k \geq 2$, que son $C P T$, la misma no es suficiente para obtener una caracterización por subgrafos prohibidos de los grafos $k$-tree que son $C P T$. Por ejemplo, el grafo $G_{\widehat{\mathbf{B}}}$ en la Figura 6.6 es un grafo 2-tree $C P T$, aunque el poset $\widehat{\mathbf{B}}$ es un subposet prohibido para los posets 2-tree CPT. El Lema 6.2.13 implica que los grafos $G_{\mathbf{B}_{1}}$ y $G_{\mathbf{B}_{2}}$ no son grafos $C P T$. En particular, $G_{\mathbf{B}_{1}}$ y $G_{\mathbf{B}_{2}}$ son prohibidos de la clase de grafos 2-tree $C P T$. Conjeturamos que son los únicos.

Conjetura 6.3.5. Sea $G$ un grafo 2-tree. $G$ es $C P T$ si y solamente si $G$ no tiene a $G_{\mathbf{B}_{1}}$ ni a $G_{\mathbf{B}_{2}}$ como subgrafos inducidos. 


\section{Capítulo 7}

\section{Conclusiones y trabajo futuro}

En la primera parte de este trabajo hemos dado una caracterización de los vértices extremos de los grafos CI usando una familia auto-complementaria de subgrafos inducidos prohibidos. Hemos estudiado a la clase de grafos que son $C I$ porque ella ha sido ampliamente estudiada y está contenida en la clase de grafos $C P T$. Sin embargo, no hemos podido usar los resultados obtenidos en el estudio de los grafos o posets CPT.

A partir del Capítulo 4 nos ocupamos en el estudio de los grafos $C P T$ y posets $C P T$. Como consecuencia de una condición necesaria para ser poset $C P T$ obtuvimos una familia de subposets prohibidos minimales de los posets que son $C P T$. También mostramos diferentes ejemplos de posets que no son $C P T$ aún cuando satisfacen la condición necesaria. Demostramos que en la clase de posets $C P T$ la dimensión y la dimensión de intervalos no están acotadas superiormente.

Por otra parte, introdujimos las definiciones de posets fuertemente-CPT y dualmente$C P T$. Hemos encontrado ejemplos en cada clase descripta en el diagrama de la Figura 7.1, pero no tenemos un ejemplo que haga diferencia entre las clases dualmente- $C P T$ y fuertemente- $C P T$. En particular, hemos demostrado que todo poset cuyo grafo de comparabilidad es un árbol es fuertemente- $C P T$. 


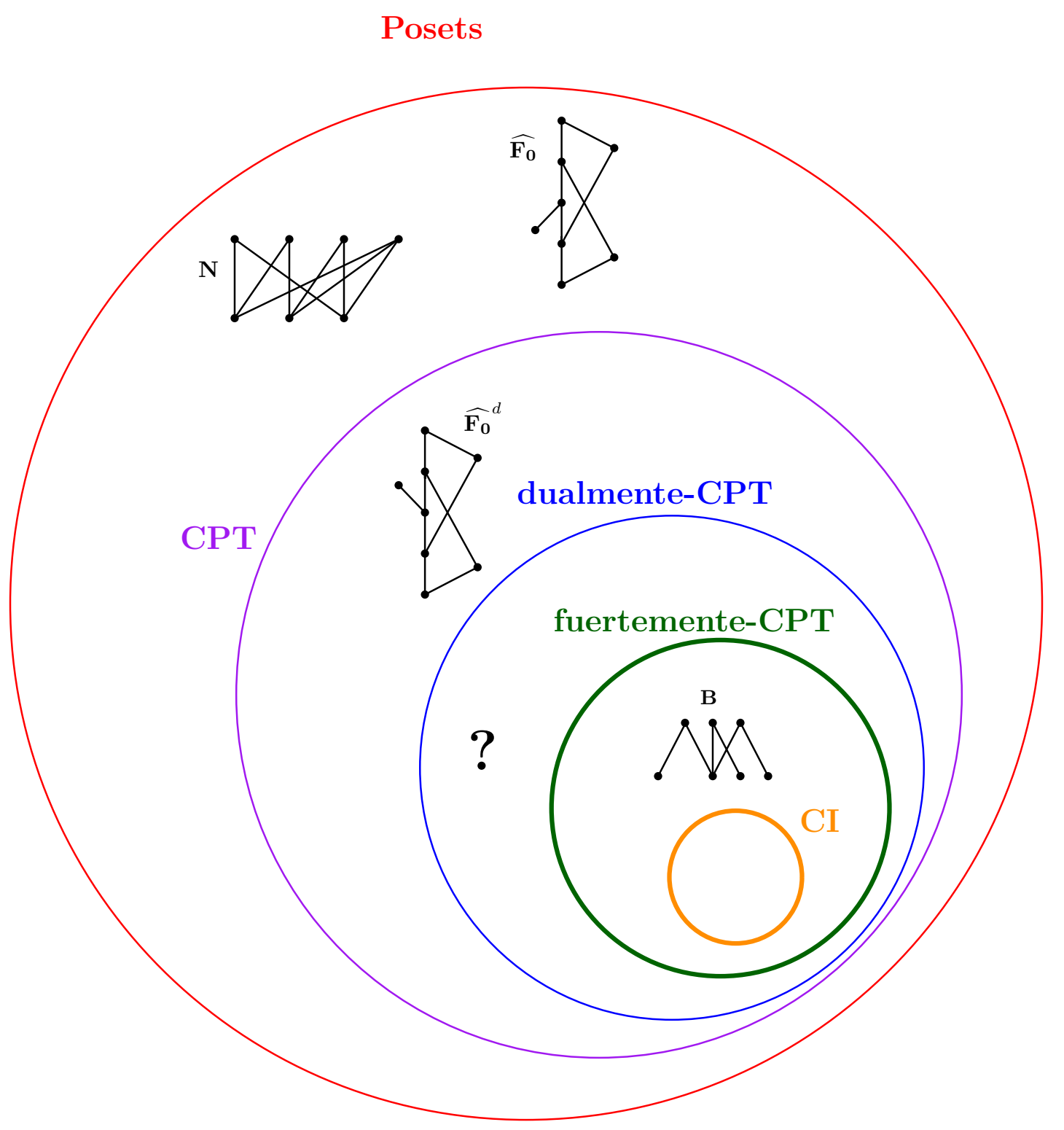

Figura 7.1: Representación de las diferentes clases de posets $C P T$ estudiadas. 
En los Capítulos 5 y 6 hemos estudiados dos clases de posets: la clase de posets $k$-tree que son $C P T$ y la clase de posets split que son $C P T$. Para ambas clases hemos demostrado que la condición necesaria para ser un poset $C P T$ dada en el Lema 4.1.2 es también suficiente. Usando este resultado damos una caracterización por subposets prohibidos de los posets split $C P T$ y de los posets $k$-tree que son $C P T$. Para ambas clases de posets mostramos que los posets dualmente- $C P T$ son exactamente los posets fuertemente- $C P T$; y dimos una caracterización por subposets prohibidos. Como consecuencia, obtuvimos una caracterización por subgrafos inducidos prohibidos tanto de los grafos que son split fuertemente- $C P T$ como de los grafos que son $k$-tree fuertemente- $C P T$. Paralelamente mostramos que los grafos split (o $k$-tree) que son dualmente- $C P T$ son exactamente los grafos split (o $k$-tree) que son fuertemente- $C P T$. Por otra parte, observamos que aunque el Teorema 5.1.5 nos brinda una caracterización por subposets prohibidos de los posets split que son $C P T$, la misma no es suficiente para obtener una caracterización por subgrafos prohibidos de los grafos split que son $C P T$. Una situación similar se observa para los grafos $k$-tree que son CPT.

Además, se plantearon problemas que han quedado abiertos:

- Problema abierto 4.2.4: ¿Existe un poset dualmente-CPT que no sea fuertemente- $C P T$ ?

- Problema abierto 4.2.5: ¿La propiedad de ser fuertemente-CPT es hereditaria por subposets?

- Problema abierto 4.3.5: Determinar si la dimensión de los posets dualmente$C P T$ está acotada superiormente por una constante. La misma pregunta para los posets fuertemente- $C P T$.

En un trabajo futuro comenzaremos a profundizar en estos problemas utilizando descomposición modular. 
De los resultados obtenidos en esta tesis surgieron los trabajos [2], enviado para publicar en Discrete Applied Mathematics, y [3], enviado para publicar en Discussiones Mathematicae Graph Theory.

El interés despertado en otros grupos de investigación por los resultados obtenidos en este trabajo se reflejan en la invitación a dictar una conferencia sobre los mismos durante el 16th Haifa Workshop on Interdisciplinary Applications of Graphs, Combinatorics and Algorithms que se llevó a cabo en la Universidad de Haifa, Israel, en Junio de 2016. 


\section{Bibliografía}

[1] L. Alcón, N. Gudiño, M. Gutierrez, On containment graphs of paths in a tree, Electronic Notes in Discrete Mathematics, Volume 50, (2015), pp 175-180.

[2] L. Alcón, N. Gudiño, M. Gutierrez, Introducing containment graphs of paths in a tree, Discrete Applied Mathematics. Eviado para publicar.

[3] L. Alcón, N. Gudiño, M. Gutierrez, End-vertices in containment interval graphs, Discussiones Mathematicae Graph Theory. Eviado para publicar.

[4] J. Bondy, U. Murty, Graph Theory, Springer, New York, NY, USA. (2007).

[5] A. Brandstädt, V. B. Le, J. P. Spinrad, Graph Classes: A Survey, SIAM Monographs on Discrete Mathematics and Applications. (1999).

[6] B. Dushnik, E. Miller, Partially ordered sets, American Journal of Mathematics 63, (1941), pp. 600-610.

[7] P.C. Fishburn, W.T. Trotter, Geometric Containment Orders: A survey, Order 15, (1999), pp. 167-182.

[8] T. Gallai, Transitiv Orientierbare Graphen, Acta Mathematica Hungar 18, (1967), pp. 25-66. 
[9] A. Ghouila-Houri, Caractrization des graphes non orients dont on peut les arrêtes de manire obtenir le graphe d'une relation d'order, C. R. Acad. Sci. Paris 254 (1962), pp. 1370-1371.

[10] J. Gimbel, Source in posets and comparability graphs, Order 9, pp. 361$365,(1992)$.

[11] M. Golumbic, Algorithmic graph theory and perfect graphs, (2da. edición), Elsevier, (2004).

[12] M. Golumbic, A.N. Trenk Tolerance graphs, Cambridge University Press, (2004).

[13] M. Golumbic, E. Scheinerman, Containment Graphs, Posets, and Related Classes of Graphs, Annals of the New York Acad. Sci. 555, (1989), pp. 192-204.

[14] G. B. Guenver, J. X. Rampon, Split orders, Discrete Mathematics 276 (1-3), (2004), pp. 249-267.

[15] D. Kelly, The 3-irreducible partially ordered sets, Can. J. Math. 29, (1977), pp. 367-383.

[16] S. Olariu, On source in comparability graphs, with applications, Discrete Mathematics, pp. 289-292, (1992).

[17] A. Pnueli, A. Lempel, S. Even, Transitive orientation of graphs and identification of permutation graphs, Canadian Journal of Mathematics 23, (1971), pp. 160-175. Mathematics 63, (1941), pp. 600-610.

[18] J. Spencer, Minimal Scrambling Sets of Simple Orders, Acta Mathematica Hungar, 22, (1971), pp. 349-353.

[19] J. Spinrad, Efficient Graph Representations, AMS, (2003). 
[20] E. Steinitz, Sur l'extension de l'orde partiel, Fund. Math. 16, (1930), pp. 386389.

[21] W. T. Trotter, J. I. Moore, Characterization problems for graphs, partially ordered sets, lattices, and families of sets, Discrete Mathematics 16, (1976), pp. 361-381.

[22] W. T. Trotter, J. I. Moore, D. P. Sumner, The dimension of a comparability graph, Proceedings of the American Mathematical Society 60,(1976), pp. 35-38.

[23] W. Trotter, Combinatorics and Partially Ordered Sets: Dimension Theory, Ann. (1992).

[24] W. Trotter, Stacks and Splits of Partially Ordered Sets, Discrte Mathematics 35, Issues 13, (1992), pp. 229-256.

[25] J. Urrutia, Patial Orders and Euclidean Geometry, Algorithms and Order, I. Rival, ed., Kluwer Acaddemic, Dordrecht (1989), pp. 327-346. 